\title{
Focused R\&D for Electrochromic Smart Windows: Significant Performance and Yield Enhancements
}

Type of Report

Reporting Period Start Date: $\quad$ September 30, 2001

Reporting Period End Date:

Principal Authors

Date Report was Issued:

DOE Award Number:

Submitting Organization:
Final

April 30, 2004

Dr. Mark Burdis

Dr. Neil Sbar

September $23^{\text {rd }} 2004$

DE-FC26-01NT41259

SAGE Electrochromics, Inc 2150 Airport Drive

Faribault, MN 55021

(507) 333-0078

www.sage-ec.com

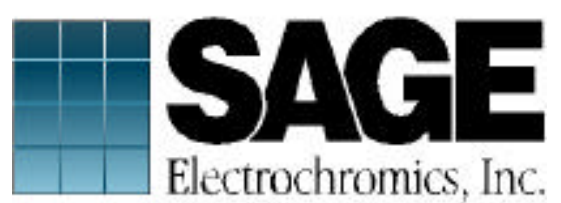




\section{Disclaimer}

"This report was prepared as an account of work sponsored by an agency of the United States Government. Neither the United States Government nor any agency thereof, not any of their employees, makes any warranty, express or implied, or assumes any legal liability or responsibility for the accuracy, completeness, or usefulness of any information, apparatus, product, or process disclosed, or represents that its use would not infringe privately owned rights. Reference herein to any specific commercial product, process, or service by trade name, trademark, manufacturer, or otherwise does not necessarily constitute or imply its endorsement, recommendation, or favoring by the United States Government or any agency thereof. The views and opinions of authors expressed herein do not necessarily state or reflect those of the United States Government or any agency thereof." 


\section{Table of Contents}

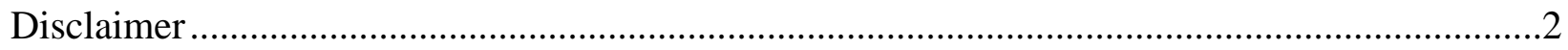

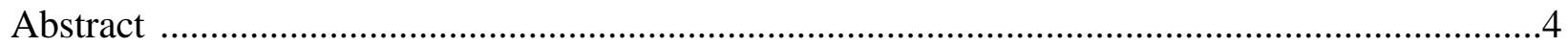

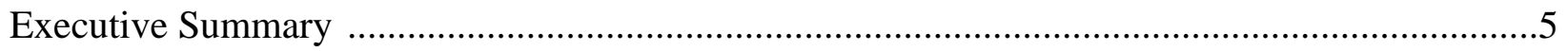

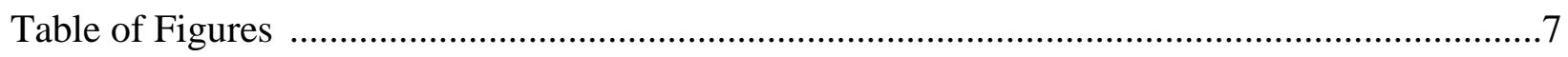

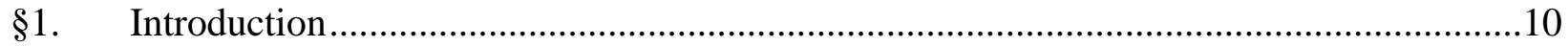

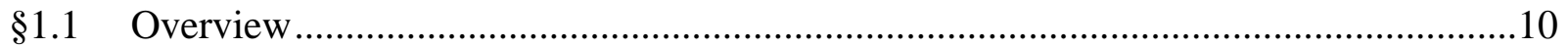

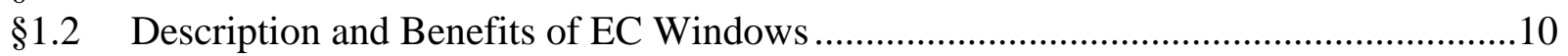

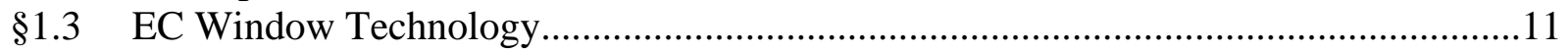

$\$ 1.4 \quad$ Background to Proposal ......................................................................................13

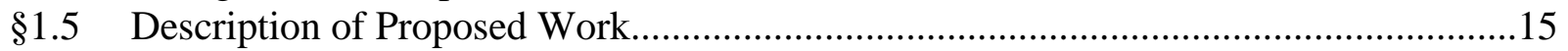

\$1.6 Project Objectives and Maturation Stages ................................................................16

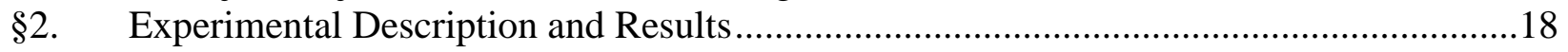

$\S 2.1 \quad$ Task 1 - Materials development ...........................................................................18

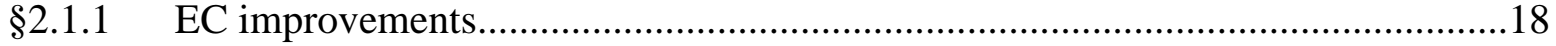

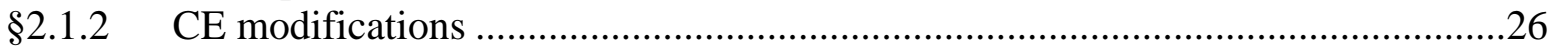

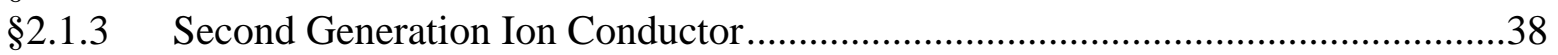

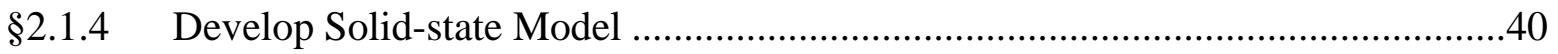

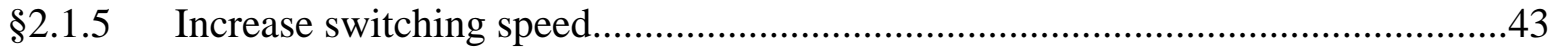

\$2.1.6 Summary of Improvements made to Materials Properties ....................................44

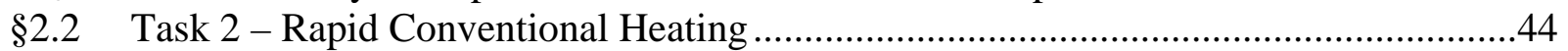

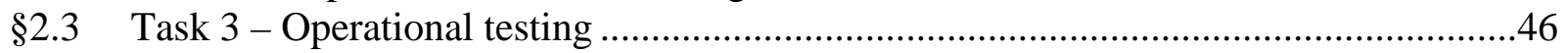

\$2.3.1 Design and Develop Prototype Test Station ..................................................4

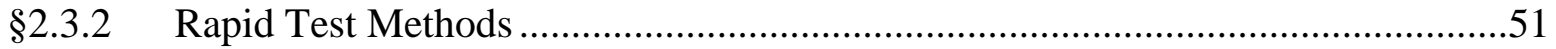

\$2.3.3 Electro-optical Device model ............................................................................51

$\$ 2.3 .4$ Manufacturing Process Improvements ...............................................................5

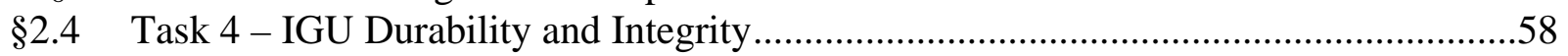

$\$ 2.4 .1 \quad$ Optimum Electrical Connection …………….......................................................5

\$2.4.2 Development of a Lower Cost Method for Bus-Bar Deposition ............................59

$\$ 2.4 .3 \quad$ Develop tests to ensure IGU Durability........................................................62

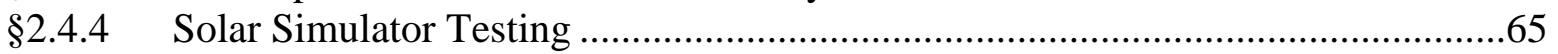

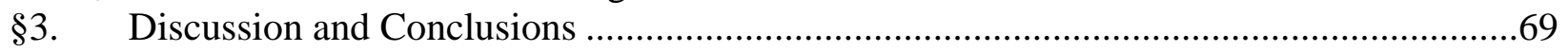

$\$ 3.1 \quad$ Achievement of Project Objectives .........................................................................

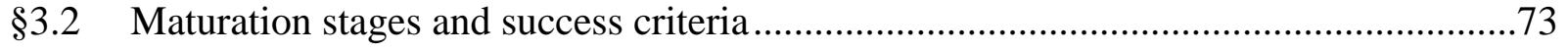

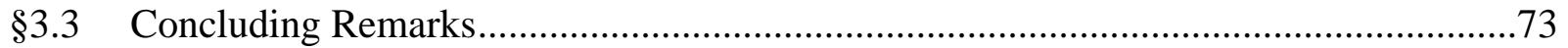

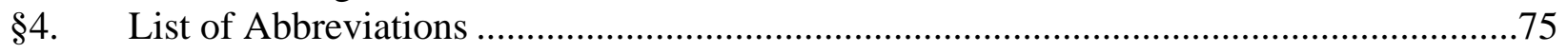

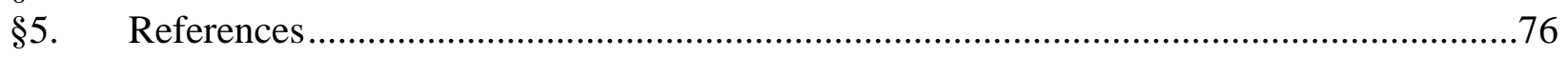

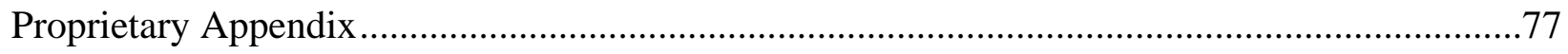




\section{Abstract}

Developments made under this program will play a key role in underpinning the technology for producing EC devices. It is anticipated that the work begun during this period will continue to improve materials properties, and drive yields up and costs down, increase durability and make manufacture simpler and more cost effective. It is hoped that this will contribute to a successful and profitable industry, which will help reduce energy consumption and improve comfort for building occupants worldwide.

The first major task involved improvements to the materials used in the process. The improvements made as a result of the work done during this project have contributed to the enhanced performance, including dynamic range, uniformity and electrical characteristics.

Another major objective of the project was to develop technology to improve yield, reduce cost, and facilitate manufacturing of EC products. Improvements directly attributable to the work carried out as part of this project and seen in the overall EC device performance, have been accompanied by an improvement in the repeatability and consistency of the production process.

Innovative test facilities for characterizing devices in a timely and well-defined manner have been developed. The equipment has been designed in such a way as to make scaling-up to accommodate higher throughput necessary for manufacturing relatively straightforward.

Finally, the third major goal was to assure the durability of the EC product, both by developments aimed at improving the product performance, as well as development of novel procedures to test the durability of this new product. Both aspects have been demonstrated, both by carrying out a number of different durability tests, both in-house and by independent thirdparty testers, and also developing several novel durability tests. 


\section{Executive Summary}

This project has addressed several aspects of EC window production, with a particular emphasis on manufacturability. The content of the program was wide ranging, incorporating microscopic studies of defect mechanisms, as well as device testing, hardware development, quality programs, and theoretical modeling. Many useful things have been learnt as a result of carrying out this project. For example, a significant understanding of microscopic defect mechanisms, particularly regarding the formation of a defect resulting in clear non-coloring areas has been achieved. Also, as another example, impressive durability results have been obtained for the EC IGU.

The following summarizes the high level results from each of the tasks in turn, highlighting the major achievements for each.

\section{Task 1: Materials Development}

- Investigated the electrochemistry of some particular mixed oxide thin films and found them to be acceptable for EC device fabrication;

- Successfully deposited EC films from a ceramic target, but could not fabricate a functioning device using such films. However, this work did provide a valuable insight into the EC film properties required for working EC devices;

- Produced functional EC devices using lower deposition temperatures;

- Investigated different metallic ratios in the $\mathrm{CE}$, and increased the understanding of device behavior and dependence on CE properties;

a Thoroughly researched the effect of poorly controlled process conditions, such as background gas levels, substrate temperature prior to deposition, magnetic array strength and uniformity, etc., on the properties of the CE films in particular, resulting in dramatically improved repeatability and therefore improved quality for the process;

- Produced second generation ion conductors with dramatically improved performance;

口 Developed a solid-state model of the EC device;

- Improved the understanding of the rate limiting factors for switching speed in the EC device.

\section{Task 2: Process Improvement}

- Developed improved thermal processing which eliminated the 'rib' defect;

口 Dramatically improved uniformity of heating.

\section{Task 3: Operational Testing}

- A custom EC test station has been designed, built and the control software written. The tester allows measurements of electronic and optical performance of multiple devices simultaneously, and is networked so data added to a database can be remotely accessed and analyzed;

a An electro-optical device model has been developed, as the first step towards allowing extraction of a set of parameters to calibrate a device controller; 
- Design of Experiments methodology has been introduced across the entire company in order to maximize the benefits of the limited experimental time which is available;

Task 4: IGU Durability and Integrity

- A low-cost, highly reliable electrical feedthrough system has been developed to enable the power to reach the EC device without compromising the IGU seal;

- Methods for investigating a lower cost bus-bar deposition process have been pursued, with limited success. Attempts to produce an electroplated bus-bar have been unsuccessful, but direct dispense methods show promise, not only for lowered costs, but also for producing lower defect levels;

- Tests to measure the durability of the IGU have been developed;

口 $\quad$ The SageGlass ${ }^{\circledR}$ EC IGU have demonstrated excellent durability to a wide variety of physical, electrical and chemical stresses;

The overall objective of the project was to demonstrate the viability of EC technology for energy efficient architectural glazing applications. It was focused on improving the EC performance durability, increasing the manufacturability and therefore the yield, thereby reducing the cost to allow high market penetration. Achieving these goals will accelerate the widespread acceptance of EC technology in buildings. More specifically, the scope was to:

- Develop and implement new materials technologies to improve EC glazing performance and enable broader penetration of both residential and commercial markets;

- Develop new EC process technologies to increase yield and throughput, resulting in reduced manufacturing cost;

- Assure EC reliability and durability by developing robust fabrication methods and implementing extensive environmental testing of both the EC IGU and the thin-film device.

Each of these objectives has been largely achieved. As a result, the next phase of SAGE development is currently being implemented: a transition from pilot-scale production to a full-scale manufacturing operation. 


\section{Table of Figures}

Figure 1. 1 - Schematic of the principle of operation of a SageGlass ${ }^{\circledR}$ EC window.................. 12

Figure 1. 2 - The SageGlass ${ }^{\circledR}$ electrochromic device ............................................................. 13

Figure 2. 1 - (a) 2-dimensional and (b) 3-dimensional AFM plots of pilot line EC film. Roughness $\left(R_{a}\right)$ values are on the order of $13 \mathrm{~nm}$ with a peak-to-valley distance of $230 \mathrm{~nm}$.

Figure 2. 2 - (a) 2-dimensional and (b) 3-dimensional AFM plots of EC films produced from the ceramic target. Roughness $\left(R_{a}\right)$ values $(9 \mathrm{~nm})$ are slightly lower than for the standard films, whereas the peak-to-valley distance $(80 \mathrm{~nm})$ appears significantly lower than for the pilot line film.

Figure 2. 3 - XRD comparison of pilot line (black) and ceramic target (blue) EC films. The ceramic target data suggests that the films are amorphous; the pilot line film pattern suggests a crystalline film. The large hump in the data from the pilot line sample centered at around $24^{\circ} 2$-theta is a combined triplet from the monoclinic (200), (020) and (002) peaks. Crystallite size determination from diffraction pattern modeling suggests an average of 5$10 \mathrm{~nm}$. The strong diffraction peaks at $\sim 26.5,34$ and $38^{\circ}$ 2-theta are from the underlying conductive oxide film.

Figure 2. 4 - Electrochemical measurements of a CV of a sample of CE-II in a lithium containing electrolyte. Sweep rate was $20 \mathrm{mV} / \mathrm{s}$ and the sample area was $4 \mathrm{~cm}^{2}$. The open circuit potential was approximately $3.1 \mathrm{~V}$ vs. $\mathrm{Li} / \mathrm{Li}^{+}$

Figure 2. 5 - Variation in CE film properties shown as a function of base pressure for films deposited in the pilot line sputtering machine. All films deposited under the same conditions.

Figure 2. 6 - Effect of argon addition on the deposition rate and optical absorption of CE films deposited in the lab coater. Conditions for the films deposited with argon added are given in the text.

Figure 2.7 - Effect of water addition on the deposition rate and optical absorption of CE films deposited in the lab coater. Conditions for the films deposited with water added are given in the text. A wet oxygen flow of $60 \mathrm{sccm}$ corresponds to approximately 6000ppm.

Figure 2. 8- The thickness dependence of films grown with different levels of nitrogen contamination. In addition, several films deposited at elevated temperature (red circles) and two films deposited with $\mathrm{CO}_{2}$ addition (blue triangles) are also shown. Note there is no dependence of thickness on either $\mathrm{N}_{2}, \mathrm{CO}_{2}$ or heating.

Figure 2.9- Effect of adding nitrogen to the sputtering gas on the measured absorption for a number of thin films. The values for $\mathrm{CO}_{2}$ are also shown for comparison.

Figure 2. 10 - The variation of transmission (relative) as a function of run number showing a significant process variation. The number of passes is also indicated, showing that there is some correlation, but this is insufficient to explain the variation seen here. Each "string" of joined points represents one run, in most cases there are six points..... 
Figure 2. 11 - The base pressure of the coater prior to $\mathrm{CE}$ deposition as a function of time, plotted alongside the measured device transmission after CE deposition. The number of passes across the target is also shown as this will alter the thickness of the CE film, and hence the measured transmission.

Figure 2. 12 - Transmission of devices measured after CE deposition as a function of initial substrate temperature.

Figure 2. 13 - Plot of internal voltage versus current (diamonds) and OD (squares) for a typical large area EC device. At lower voltages, the current density is low, and the OD increases fairly rapidly, but above an internal voltage of approximately $2 \mathrm{~V}$, the current density rapidly increases and the optical density saturates. The threshold voltage is the intercept of the I-V characteristic on the voltage axis.

Figure 2. 14 - An example of electronic breakdown leading to saturation of the optical density of an EC device.

Figure 2. 15 - Example of electronic behavior of EC/CE junction, showing clear evidence of rectification in both forward and reverse direction.

Figure 2. 16 - Intercept voltages plotted against number of passes of $\mathrm{CE}$ (equivalent to $\mathrm{CE}$ thickness) showing little or no variation.

Figure 2. 17 - An example of a CV taken from an EC device made with no IC layer. Note the Ohmic behavior of the device, and poor coloration.....

Figure 2. 18 - An example of the defect known as 'ribs'

Figure 2. 19 - The updated monolithic device tester, shown configured to measure two $46 \times 89 \mathrm{~cm}(18 \times 35 ")$ samples. The backlight is turned off for the photograph.

Figure 2. 20 - The user interface of the device tester, showing the wide range of measurements available, including current, voltage, transmission, color and even temperature.

Figure 2. 21 - Schematic figure of heating and cooling set-up for the device tester.................. 50

Figure 2. 22 - Test Dialog Box ......................................................................................... 51

Figure 2. 23 - Device Schematic Model (Equivalent Circuit) ................................................. 53

Figure 2. $24-100 \times$ photomicrograph of a $>2 \mathrm{M} \Omega$ isolation circle scribed in the first transparent conducting oxide (TCO).

Figure 2. 25 - Photomicrograph of laser patterning through the entire EC stack. The surrounding films could be colored and bleached independently of the isolated circle, which remained bleached. The left is at a magnification of $100 \times$, while the right is taken at $1000 \times$.

Figure 2. 26 - Small (3.2 cm (1.25”) diameter) EC devices fabricated using directly dispensed bus-bars. The bus bars are indicated by the arrows.

Figure 2. 27 - Measurements of seal temperatures of a SageGlass ${ }^{\circledR}$ IGU in the outdoor solar soaker, plotted as a function of the center-of-glass (COG) temperature of the EC lite. Note that even for temperatures of $90^{\circ} \mathrm{C}$, the seal temperatures only reach about $60^{\circ} \mathrm{C}$, suggesting that even for these high exposures the seals should be capable of surviving. 65

Figure 2. 28 - The XR260 test chamber at NREL 
Figure 2. 29 - Charge discharge curves for one of the SageGlass ${ }^{\circledR}$ IGUs subjected to simulated solar soaking at NREL, measured at various stages of cycling. It is apparent that there is very little change in the characteristic as a result of the solar soaking.

Figure 2. 30 - Transmission as a function of wavelength for one of the devices subjected to simulated solar soaking at NREL measured at the start of the test, and after 80000 cycles. Once again, it is clear there is very little change as a result of the testing. 


\section{§1. Introduction}

\section{\$1.1 Overview}

This document contains details of the work done by SAGE Electrochromics, Inc. between October 2001 and March 2004 under DOE award number DE-FC26-01NT41259. The work addressed the subject of increasing yields and improving the performance of existing SageGlass ${ }^{\circledR}$ electrochromic windows. The document is split into two sections, with the body of the document composed of the main narrative, with the proprietary details in an appendix contained within a second separate document.

During the course of the project, the initial proposal was modified to allow some further areas to be addressed. The additional tasks introduced at this stage have simply been included in the most appropriate task area, which has the effect of making the document more contiguous and less 'chopped-up'. For this reason, the details of the original task numbering are not used here and so the section numbers do not correspond exactly to that outlined in the initial and continuation proposals. However, the main task headings are retained.

The report is organized as follows: First, the subject of electrochromic technology is briefly discussed, and the work placed into context, and then each task is treated in some detail. The main report is then split into several sections addressing the various task areas. These include: (1) Materials Development, (2) Process Improvement, (3) Operational Testing, and (4) Insulated Glazing Unit (IGU) Durability and Integrity. Finally, there is a discussion of how well the objectives of the project were met, and the general conclusions of the work.

\section{\$1.2 Description and Benefits of EC Windows}

There is a need to improve the energy efficiency of building envelopes as they are the primary factor governing the heating, cooling, lighting and ventilation requirements of the buildings themselves - influencing $53 \%$ of building energy use. ${ }^{1}$ In particular, windows contribute significantly to the overall energy performance of the building 'envelope', thus there is a need to develop advanced energy efficient window and glazing systems.

The electrochromic (EC) window represents the next generation of advanced glazing technology that will both reduce the energy consumed in buildings, as well as improve both the overall comfort of the occupants and the thermal performance of the building envelope. "Switchable" EC windows provide, on demand, dynamic control of visible light, solar heat gain, and glare without ever blocking the view. ${ }^{3}$ As exterior light levels change, the performance of the window can be electronically adjusted to suit conditions. A schematic diagram illustrating how SageGlass ${ }^{\circledR}$ electrochromic windows work is shown in Figure 1. 1. In the 'clear state', depicted in the uppermost diagram, both solar heat and visible light are allowed to pass through the window. In this condition, the window is practically indistinguishable from a 'normal' window glazing, typically permitting upwards of $60 \%$ of the direct visible light to be transmitted. This 'clear' condition allows the building occupant to maximize the natural daylight and heat flowing through the window, thereby reducing the requirements for lighting and heating.

However, should the need arise, it is possible for the building occupant to reduce the level of transmitted light from the clear state, in order to reduce the visible light and solar heat gain into 
the building. A typical dark state visible transmission for a SageGlass ${ }^{\circledR}$ window is around $3 \%$. This is accomplished by electrochemically inducing absorption in the thin film stack, and the absorbed energy is thermalized and then re-radiated to the outside as a result of a low-e surface, which is an intrinsic part of the EC device.

The ability to block solar energy is particularly useful in warmer climates where cooling requirements dominate, as it will reduce the overall load on the cooling systems. It should be noted that control over solar heat gain will also result in the use of smaller and therefore more economical air conditioning equipment. In addition, the reduction in visible light gain can be used to reduce glare in the office environment - useful for 'knowledge workers', and especially those using computers. It should be noted that the transmission can be held at any point between fully dark and fully clear, and that this is a fairly simple matter of electronic control, and that the coloration and bleaching are totally reversible.

Control of EC windows is highly flexible. They can be arranged to be part of a totally automatic integrated building energy management system, or can be individually controlled by users. EC windows operate using very low levels of electrical power. Typical voltages are between 1 and $3 \mathrm{~V}$, with currents of less than $1 \mathrm{Am}^{-2}$, and these requirements can be met from various sources, including photovoltaic cells.

As is obvious from the preceding discussion, SageGlass ${ }^{\circledR}$ EC glazing offers huge potential in cooling and lighting cost reductions, with the added benefit of improving thermal and visual comfort. ${ }^{4}$ This will lead to a reduction in the energy consumption, and thereby reduce the US dependence upon foreign oil imports.

However, if the required step change in the energy efficiency and performance of buildings is to be achieved, there is a clear need to bring EC technology to the marketplace. This project addresses the need to accelerate the widespread introduction of EC windows in buildings and thus maximize total energy savings in the US and worldwide.

\section{\$1.3 EC Window Technology}

The SageGlass ${ }^{\circledR}$ EC device is a series of thin films deposited onto a glass substrate one on top of the other to form a stack. ${ }^{5}$ This is shown in Figure 1. 2, and is known as an 'all solid-state ECsystem', to distinguish it from an EC window with a polymeric electrolyte - 'a polymeric ECsystem'. The all solid state system is much more difficult to produce, because any imperfections in the coating - pinholes, etc. - will lead to short circuits between the two transparent conductors, which in turn will lead to highly noticeable defects. The polymeric approach does not suffer from this problem, as a thick layer of insulating polymeric material separates the transparent conductors. 


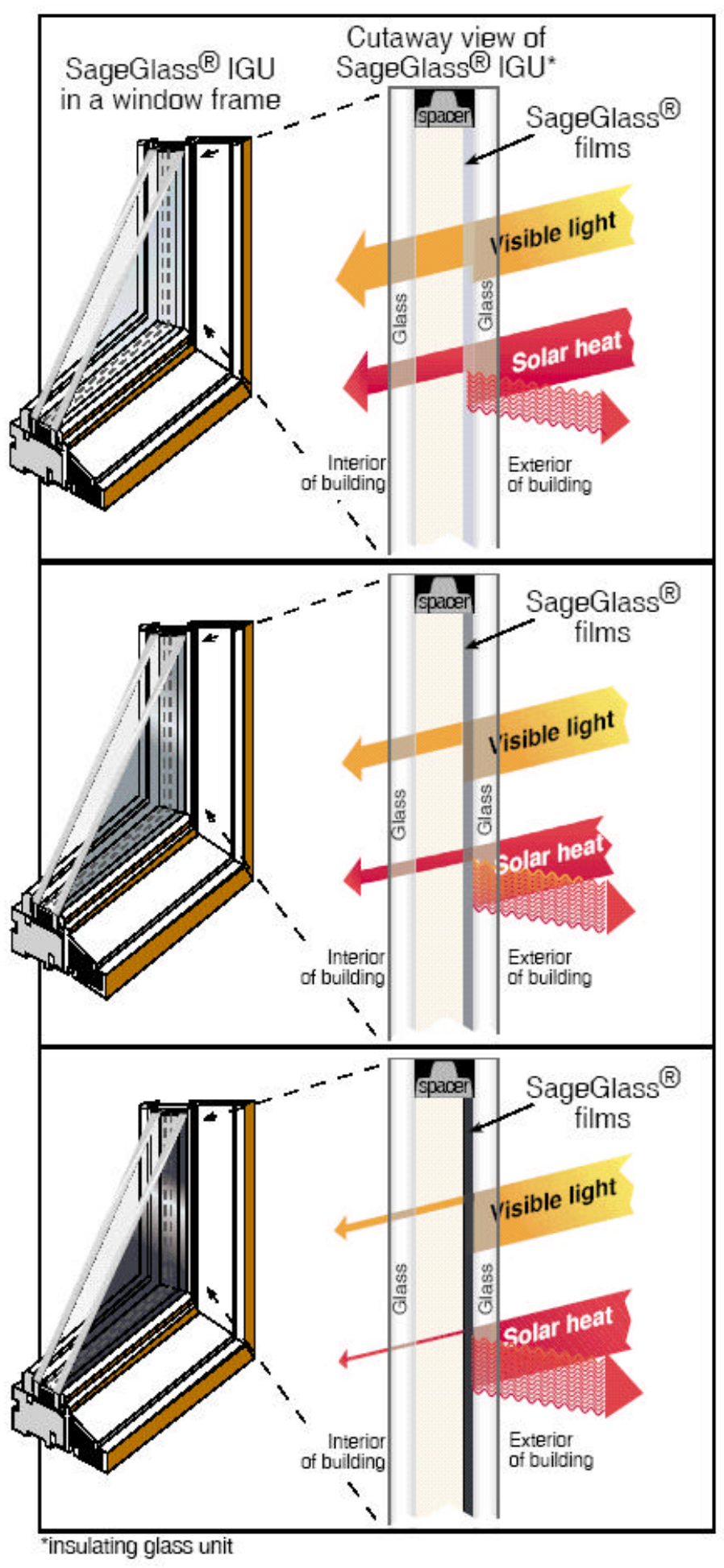

Figure 1.1 - Schematic of the principle of operation of a SageGlass ${ }^{\circledR}$ EC window.
However, the polymeric device has three significant disadvantages when compared to the all-solid state system: (1) The polymeric materials are generally unstable to solar radiation, and will therefore give very poor durability in applications where solar exposure is a possibility i.e. most window applications; (2) the polymeric IGU system requires at least ${ }^{*}$ three sheets of glass, increasing the cost, and significantly increasing the weight. The fact that there are at least three sheets of glass means that original equipment manufacturer's (OEM) framing systems need to be redesigned, giving further increases in cost; (3) the polymeric system does not provide the EC window with a low-e surface, thereby compromising the energy efficiency of the system.

The SageGlass ${ }^{\circledR}$ all solid-state EC device does not suffer from any of these issues. It is fabricated from inorganic materials, which lead to excellent durability when exposed to solar radiation, high temperatures, etc. It can be deposited onto tempered glass, giving it the required structural integrity for use in building applications, and can be fabricated into an IGU that is an almost direct replacement for a standard $\mathrm{IGU}$, requiring minimal re-engineering of the framing system. Finally, the uppermost transparent conductor acts as a low-e surface, giving it excellent thermal performance.

The outermost layers are transparent conductors (TC), which are used to apply a voltage to the active layers

\footnotetext{
* In polymeric systems, the 'laminating' polymer electrolyte is too weak to be used as a safety laminate. Many times, therefore, it is necessary to laminate a further sheet of glass to the device in order for it to have the structural integrity to be used in construction applications.
} 


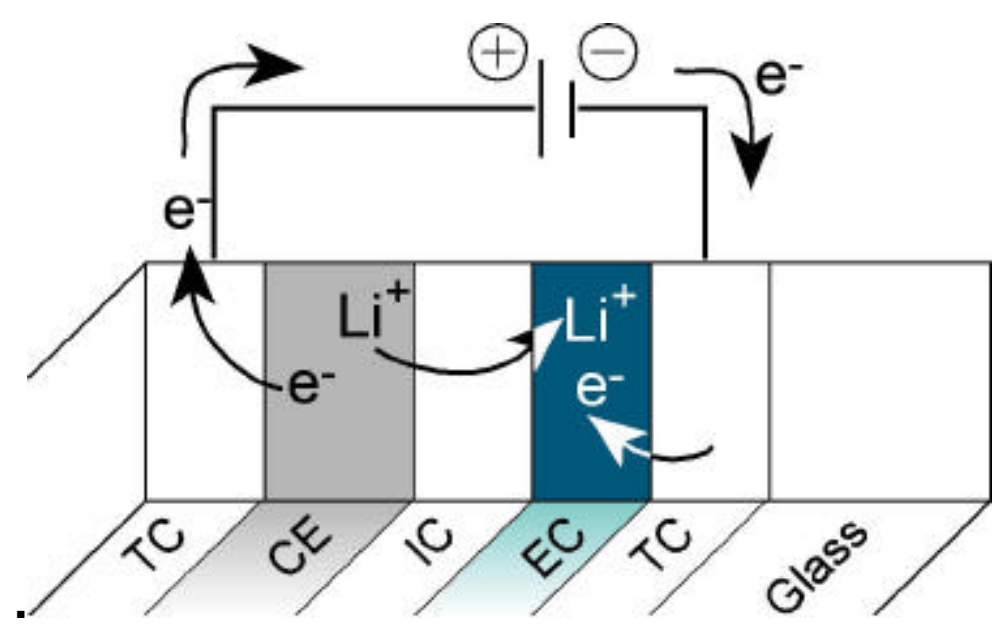

Figure 1. 2 - The SageGlass ${ }^{\circledR}$ electrochromic device that are sandwiched

between. These active layers consist of an EC layer, an ion conductor (IC) layer, and a counter electrode layer (CE). Charge, in the form of electrons and ions, is shuttled between the $\mathrm{CE}$ and the EC layers, producing the bleached and colored states respectively: The electrons are passed around the outer circuit, while the ions are transported through the IC. Insertion of charge into the EC layer will cause that layer to color to a depth depending upon the amount

of charge transferred. The effect is completely reversible, and is accomplished simply by reversing the polarity of the voltage. The polarity for coloration is shown in Figure 1. 2.

There are several terms that will be used throughout the report, and it is appropriate that these are defined. Table 1. 1 summarizes some of the more commonly used terms. Acronyms are defined in Section $\S 4$ on page 75 . Many additional terms are defined in footnotes throughout the text.

\section{\$1.4 Background to Proposal}

SAGE Electrochromics Inc., (SAGE) has been exclusively developing an all-solid state EC device for over a decade, and prior to the beginning of this project, had entered a Pilot Line phase, which was designed to scale-up the technology developed in the laboratory to allow production of limited quantities of 'full-size' EC windows. The Pilot Line consists of a sputter coater capable of depositing thin films over an area of approximately $1 \mathrm{~m} \times 1 \mathrm{~m}(40$ " $\times 40$ "), a clean-room used for several other glass processing operations, and some testing and IGU fabrication facilities.

The Pilot Line was intended to allow investigation of the issues and problems associated with scale-up, and would thereby allow the first full-scale manufacturing plant to be designed and built with a high level of confidence.

This proposal was made after the EC device deposition process had been initially transferred from the laboratory scale onto the Pilot Line. It was made at a time when SAGE was in the early stages of producing large area EC glazing, and the content of the proposal therefore reflects some of the attendant issues related to improving performance over that achieved at that time, and also to improve the overall process yield. The tasks covered a wide range of disciplines, from constructing electrochemical models of the device through to understanding the durability of the finished glazing product when exposed to realistically anticipated environmental stresses. 
At the time of the proposal, the visible transmission of the SageGlass ${ }^{\circledR}$ window ranged from $\sim 4 \%$ in the darkened state to around $60 \%$ in the clear state. In addition, the color of the device was not optimized with a dark blue hue when colored and a slight tinge of yellow at maximum transmission. Architects and end-users (in focus groups) had stated a strong preference for a neutral colored window that switches from dark gray to perfectly clear. A more neutral color and enhanced transmission in the clear state would broaden accessibility to all architectural markets.

A second issue was the cost of the SageGlass ${ }^{\circledR}$ window. Initial yields on the pilot line were low and there were a number of bottlenecks in the processing operations. It was obviously important to increase yields and expedite manufacturing steps because the higher the yields and the faster products can move through fabrication operations, the lower the costs will be. Some materials and process choices, while capable of producing limited quantities of high quality devices, had yield and throughput issues that had to be overcome. For example, there are a number of heating steps in the fabrication processes. Some of these contribute to the exceptional durability of the product, but in other cases the problems of heating uniformly could introduce variability in the appearance of the device. In addition some heating steps are very time consuming and reduce throughput. Where appropriate, it was proposed to reduce or eliminate some heating, and in other cases try to institute rapid thermal processing.

Another important area is the final testing of the completed electrochromic window. This is a "smart" appliance, and it is

\begin{tabular}{|l|l|}
\hline Term & Definition \\
\hline $\begin{array}{l}\text { Bleached State } \\
\text { Transmission }\left(\mathrm{T}_{\mathrm{ble}}\right)\end{array}$ & $\begin{array}{l}\text { The integrated visible transmission } \\
\text { of the device when it is in the clear } \\
\text { state. }\end{array}$ \\
\hline $\begin{array}{l}\text { Colored State } \\
\text { Transmission }\left(\mathrm{T}_{\mathrm{col}}\right)\end{array}$ & $\begin{array}{l}\text { The integrated visible transmission } \\
\text { of the device when it is in the } \\
\text { colored (or dark) state. }\end{array}$ \\
\hline Dynamic Range & $\begin{array}{l}\text { The value } \mathrm{T}_{\text {ble }} / \mathrm{T}_{\text {col }} \text {. This is a figure } \\
\text { of merit for the device }- \text { the higher } \\
\text { the dynamic range, the better the } \\
\text { device. }\end{array}$ \\
\hline Leakage Current & $\begin{array}{l}\text { The electronic current flowing } \\
\text { through the device. It is the ionic } \\
\text { current that leads to coloration, so } \\
\text { leakage current should be } \\
\text { minimized. }\end{array}$ \\
\hline Optical Density (OD) & $\begin{array}{l}\text { The logarithm of the dynamic } \\
\text { range. }\end{array}$ \\
\hline Monolithic & $\begin{array}{l}\text { The description of the device } \\
\text { before fabrication into an IGU }\end{array}$ \\
\hline
\end{tabular}

Table 1. 1 - Some commonly used terms and their definitions necessary to measure the optical and electrical performance of each one to determine whether or not it meets the required specifications. Final testing is currently a lengthy process because each device is slowly cycled through the total switching range as optical measurements are made. Rapid testing based on measuring a few key device parameters was proposed to qualify and calibrate window systems more quickly.

Furthermore, architects, building owners, and end-users require information about the durability of the EC window and its reliability over time. There was particular concern about the IGU seal and interconnections for control and power. The EC IGU differs from conventional windows in that interconnections to power the device must pass through the moisture barrier seal. Interconnects and feedthroughs that preserve the seal integrity had to be developed. Most importantly, definitive and rigorous evaluations of seal durability were proposed to assure customers that the IGU for an EC window could meet the performance standards of conventional 
IGUs. There was a similar concern regarding the durability of the EC film stack when exposed to the range of solar and environmental stresses that a window experiences over its lifetime. It was proposed to develop accelerated tests that could quickly subject window units to relevant high stresses so that failures would be revealed in a relatively short time.

These major issues formed the basis of the proposal, and the tasks were designed to address these areas.

\section{$\$ 1.5$ Description of Proposed Work}

This section contains a brief summary of the proposed work that was outlined in the proposal. Each task reflects a broad area of work that was necessary to improve the device performance and increase the yield as described in the previous section.

Task 1: Materials Developments - Each of the active electrochromic layers was to be optimized with respect to device optical performance and ease of processing, with the broad aim of improving device neutrality and increasing the dynamic range. The basic approach to evaluating each alternative sputtered material and/or composition included fabricating a small sputtering target for use on the laboratory MRC coaters. Films were to be deposited and analyzed using a variety of techniques with support from The Center for Interfacial Engineering at the University of Minnesota ${ }^{\dagger}$. Experiments on solution deposited films were to be performed with help from the Center for Microengineered Materials at the University of New Mexico ${ }^{\ddagger}$. Most of the work was to be carried out to determine the impact of materials properties on the color of the device - with the goal of achieving better color neutrality, both in the bleached (or clear) state, and in the colored state. An additional focus was placed on materials and process changes to increase the dynamic range and optimize switching performance. If desirable film properties could be achieved on the lab scale, full size pilot line targets and processing would be implemented for the production of large area windows.

Task 2: Rapid Thermal Processing - The objective of this task was to develop rapid thermal processes to reduce the anticipated bottlenecks during production. These processes were also required to be highly uniform, resulting in high quality, durable EC devices, and fully compatible with the proposed production process.

Task 3: Process Improvement - The objective of this activity was to optimize the processing parameters to achieve the best device performance. However, device fabrication through pilot line coating operations is a multivariate process and the variables are not always independent, meaning that interactions between variables can become important. Consequently, Design of Experiments (DOE) techniques were to be implemented to identify the key processing variables, among the multiple fabrication steps, that had the most impact on performance, yields and throughput. Process optimization was then to be carried out, with the aim of achieving high throughput processing of high quality EC devices.

Additionally, a solid-state model was to be developed to explore the electronic behavior of SageGlass ${ }^{\circledR}$ devices. It had been found that good devices have a leakage characteristic similar to

\footnotetext{
${ }^{\dagger}$ Center for Interfacial Engineering, University of Minnesota, 100 Union Street SE, Minneapolis, MN 55455

${ }^{\ddagger}$ UNM/NSF Center for Micro-Engineered Materials, 203 Farris Engineering Center, Albuquerque, NM 87131-1341
} 
that of a diode. The proposed work was focused on understanding the contributions of the various device layers and interfaces to this diode effect. The goal was to understand the device and to enable the most efficient switching regime for fast and uniform switching.

Task 4: Operational Testing - Operational testing refers to post-manufacturing device testing, both immediately after production and also extended durability or reliability testing. The testing is carried out to determine whether the EC glazing meets product specifications. This is done through a combination of optical and electrical measurements. Requirements and test parameters were to be defined and a prototype test station built and evaluated. A second aspect of this task involved achieving a better understanding of voltage/current relationships so that initial dynamic measurements of device performance could be used to predict final steady state properties.

In addition, the IGU seal must continue to provide a moisture barrier over the lifetime of the window. To this end it is essential to use the highest quality materials, processes and practices to achieve the best seal possible. Another major challenge in this task was to be to develop a lowcost interconnection system for bringing power through the sealing system to the EC device. This system must resist corrosion and not compromise the moisture barrier properties of the seal. Furthermore, development and implementation of a series of tests to measure the durability of EC IGU, and if possible to generate an accelerated life test incorporating all the anticipated stresses likely to be encountered by the EC device was also required. These tests were to include temperature cycling, humidity aging, low temperature testing, and thermal shock.

\section{\$1.6 Project Objectives and Maturation Stages}

Several decision points and milestones were developed for the various tasks, and reference will be made to these in the discussion section of this report following description of the technical results.

The following table (Table 1. 2) shows a broad outline of the maturation stages for the proposed project. These follow the work through from proposal (Stage 1) through Concept Definition (Stage 2), Lab Scale Prototype (Stage 3), Pilot Line Manufacture (Stage 4) to Transfer to Manufacturing (Stage 5). The shaded areas indicate tasks that are beyond the scope of the proposal. 


\begin{tabular}{|c|c|c|c|c|}
\hline & $\begin{array}{l}\text { Maturation } \\
\text { Stage } 2 \\
\text { - Concept Def. }\end{array}$ & $\begin{array}{l}\text { Maturation } \\
\text { Stage } 3 \\
\text { - Lab scale prototype }\end{array}$ & $\begin{array}{l}\text { Maturation } \\
\text { Stage } 4 \\
\text { - Pilot Line Prototype }\end{array}$ & $\begin{array}{l}\text { Maturation } \\
\text { Stage 5 } \\
\text { - Transfer to } \\
\text { Manufacturing. }\end{array}$ \\
\hline \multicolumn{5}{|c|}{$\begin{array}{l}\text { Task I: Materials Development } \\
\text { I.1 EC Layer Improvements }\end{array}$} \\
\hline $\begin{array}{l}\text { I.1.1 Doped EC } \\
\text { Layer }\end{array}$ & & $\begin{array}{l}\text { I1.1.1-3 Lab } \\
\text { development }\end{array}$ & $\begin{array}{l}\text { I1.1.2.4-6 Pilot Line } \\
\text { Transfer }\end{array}$ & $\begin{array}{l}\text { Transfer to } \\
\text { Manufacturing }\end{array}$ \\
\hline $\begin{array}{l}\text { I.1.2 Use of Ceramic } \\
\text { target }\end{array}$ & & $\begin{array}{l}\text { I.1.2.1-2 Lab } \\
\text { development of 'cold' } \\
\text { EC layer deposition }\end{array}$ & $\begin{array}{l}\text { Go to Stage } 4 \text { - Pilot } \\
\text { Line Demo }\end{array}$ & $\begin{array}{l}\text { Transfer to } \\
\text { Manufacturing }\end{array}$ \\
\hline \multicolumn{5}{|l|}{ I.2 CE Modifications } \\
\hline $\begin{array}{l}\text { I.2.1 Optimize CE } \\
\text { Composition }\end{array}$ & & & $\begin{array}{l}\text { I.2.1.1-4 CE } \\
\text { Deposition on Pilot } \\
\text { Line with twin } \\
\text { magnetron. }\end{array}$ & $\begin{array}{l}\text { Transfer to } \\
\text { Manufacturing }\end{array}$ \\
\hline $\begin{array}{l}\text { I.2.2 Alternative } \\
\text { Materials }\end{array}$ & $\begin{array}{l}\text { I.2.2.1-2 Feasibility } \\
\text { of new materials }\end{array}$ & $\begin{array}{l}\text { I.2.2.3-5 Deposit new } \\
\text { CE films \& lab } \\
\text { prototypes }\end{array}$ & $\begin{array}{l}\text { Go to Stage } 4 \text { - Pilot } \\
\text { Line Demo. }\end{array}$ & \\
\hline \multicolumn{5}{|c|}{ I.3 Second Generation Ion Conductor } \\
\hline $\begin{array}{l}\text { I.3.1-2 Characterize } \\
\text { and Optimize current } \\
\text { IC }\end{array}$ & $\begin{array}{l}\text { I.3.1.1-2 Develop } \\
\text { techniques }\end{array}$ & $\begin{array}{l}\text { I.3.2.1-2 Lab scale } \\
\text { evaluation }\end{array}$ & $\begin{array}{l}\text { I.3.2.3 Evaluate on } \\
\text { Pilot Line devices }\end{array}$ & $\begin{array}{l}\text { Transfer to } \\
\text { Manufacturing }\end{array}$ \\
\hline $\begin{array}{l}\text { I.3.3 Improved IC } \\
\text { technology }\end{array}$ & $\begin{array}{l}\text { I.3.3.1-3 Proof of } \\
\text { Concept }\end{array}$ & $\begin{array}{l}\text { I.3.3.4 Produce lab } \\
\text { prototypes }\end{array}$ & $\begin{array}{l}\text { Go to Stage } 4 \text { - pilot } \\
\text { line demo }\end{array}$ & \\
\hline \multicolumn{5}{|c|}{ Task II and III: Process Improvements } \\
\hline $\begin{array}{l}\text { Task II: Rapid } \\
\text { Thermal processing }\end{array}$ & & & $\begin{array}{l}\text { II.1 Rapid } \\
\text { conventional heating } \\
\text { Pilot Line }\end{array}$ & $\begin{array}{l}\text { Transfer to } \\
\text { Manufacturing }\end{array}$ \\
\hline $\begin{array}{l}\text { Task III: Operational } \\
\text { testing }\end{array}$ & & $\begin{array}{l}\text { III.1-III.2 Test Station } \\
\& \text { electro-optical device } \\
\text { model \& performance } \\
\text { parameters }\end{array}$ & $\begin{array}{l}\text { III.3-III.4 Rapid test } \\
\text { methods \& Multiple } \\
\text { unit test facility }\end{array}$ & $\begin{array}{l}\text { Transfer to } \\
\text { Manufacturing }\end{array}$ \\
\hline \multicolumn{5}{|l|}{ Task IV: Durability } \\
\hline \multirow[t]{2}{*}{$\begin{array}{l}\text { Task IV: Durability } \\
\text { and IGU Integrity }\end{array}$} & & $\begin{array}{l}\text { IV.1 Connection } \\
\text { scheme development }\end{array}$ & $\begin{array}{l}\text { IV.3 New EC-IGU } \\
\text { testing }\end{array}$ & $\begin{array}{l}\text { Transfer to } \\
\text { Manufacturing }\end{array}$ \\
\hline & & & $\begin{array}{l}\text { IV.2 Lower cost bus- } \\
\text { bar development }\end{array}$ & $\begin{array}{l}\text { Transfer to } \\
\text { Manufacturing. }\end{array}$ \\
\hline
\end{tabular}

Table 1. 2 - Project objectives and maturation stages for this project.

There now follows a discussion of each of the tasks. Within each write-up, the necessity for performing the task is explained, along with an assessment of the progress made towards achieving the goals set out in the proposal. 


\section{§2. Experimental Description and Results}

The following section takes describes the work done in order to address each of the tasks. The layout follows the task structure as far as possible.

\section{\$2.1 Task 1 - Materials development}

To improve both the performance and marketability of SageGlass ${ }^{\circledR}$ products, one of the main issues was the color of the device, both in the fully darkened and in the bleached state. Currently, the bleached state coloration contains a small degree of absorption, which is manifested as a slight yellow tinge, and the colored state color is slightly blue-gray. It is highly desirable to reduce the coloration in the bleached state, and to shift the dark state color more towards neutral.

The current Section (\$2.1) discusses work done on the individual layers, namely the EC, the CE and the IC, in order to improve the optical properties of the overall device. First, the work related to EC is discussed, including work done with different target compositions and deposition parameters, then the work done to understand and improve the CE properties, again using different compositions and deposition parameters. Then there is a discussion of the work carried out on the IC.

\section{\$2.1.1 EC improvements}

Work associated with the EC carried out during this project is specifically targeted toward modification of the optical properties to produce improved coloration in the dark state.

Several other research groups ${ }^{6}$ have studied the doping of EC materials with various other elements to shift the absorption band - usually centered in the near infra-red - more into the visible to produce a more acceptable looking colored state. Several different dopants have been used. ${ }^{7,8}$ The accepted model suggests that optical transitions, while possible between different oxidation states of the ions in the pure material, are also possible between different oxidation states of the dopant ions, and between different oxidation states of dopant and host depending on the concentrations and the total amount of inserted charge. The proposed mechanism is for the electrons associated with the inserted charge to initially occupy lower energy sites associated with the dopant ions, from where transitions to empty dopant or host sites are then possible. (Note: This class of electrochromic materials colors by a mechanism called intervalence charge transfer. This involves an electron initially reducing one of the ions in the host lattice, and then 'hopping' to a nearby 'empty' ion.) The occupation of the dopant sites upon further addition of lithium continues until all the dopant sites are occupied, whereupon occupation of the host sites commences, leading to possible transitions between differently oxidized host sites, giving a much more distributed absorption and hence a more neutral coloration. Further credence is given to this model by a successful application to a completely different mixed oxide system, that of $\mathrm{V}_{\mathrm{x}} \mathrm{Ti}_{1-\mathrm{x}} \mathrm{O}_{\mathrm{y}}{ }^{9}$

The first aspect of the work discussed here addresses modifications to the color of the electrochromic layer by changing the composition. Finally, the work aimed at modifying the properties to improve the transmission in the bleached state obtained after the device is cycled is discussed. 


\section{\$2.1.1.1 Doped electrochromic material target}

A doped target was obtained for the lab coater. Thin films were deposited onto both plain glass and tin oxide coated substrates to make both electrochemical and optical measurements. The overall aim was to determine whether the EC compound would provide better neutrality for the finished device, without compromising the overall electrochemical performance.

Thin films were deposited from the mixed metal target, and the optical properties evaluated in the usual way. It was found that the sputtering process from the doped target was not very different to that from the pure target. This meant that it was possible to deposit transparent films fairly quickly. Indeed, the refractive indices for the mixed metal oxides were also within the range of those obtained from the pure EC material.

The electrochemistry of the mixed films was found to be acceptable, and once again, indistinguishable from the electrochemistry expected from 'standard' EC films. However, it was noted that the coloration for the mixed metal films was more neutral for the initial stages of coloration (i.e. from around $70 \%$ down to $25 \%$ ), than an equivalent thickness 'standard' film. It was also noted that the film became rapidly bluer as coloration proceeded, and was almost indistinguishable from the 'standard' film when fully colored. It would be of interest to obtain a target with a higher degree of doping, and repeat this work to determine whether it is possible to maintain the neutrality throughout the full coloration range.

\section{\$2.1.1.2 EC film structure and processing to control blindcharge}

There have been many references in the open literature to a phenomenon referred to as 'Blindcharge'. ${ }^{10,11}$ This is the irreversibly incorporated lithium which is not recovered from the EC film after the first color-bleach cycle, however it causes no coloration and does not seem to upset the electrochemical kinetics of the insertion process. From this perspective, it does not appear to be a problem, but in fact, variable loss of lithium from the EC device during switching makes it difficult to precisely determine the lithium titration needed for optimum dynamic range. It is clearly important to control the amount of blindcharge resident in the as-deposited EC films. Two methods for doing so are discussed below (i) Deposition of EC films from a ceramic target, and (ii) Deposition of EC films in the transition region of the sputtering characteristic.

\section{\$2.1.1.2.1 Thin film deposition from a ceramic target}

As has been mentioned previously, the current SageGlass ${ }^{\circledR}$ process utilizes an EC thin film as the electrochromic layer. This is deposited at elevated temperatures by a reactive magnetron sputtering process. This heating process is required to be highly uniform, is time consuming and therefore expensive to implement in a high throughput manufacturing operation. In addition, handling large pieces of hot glass adds an additional hazard for the operator to an already difficult process.

An elegant solution to these two problems would be to eliminate the need for heating altogether. This has been shown to be possible by using a ceramic target ${ }^{11}$, and experiments in the lab have shown that EC films with acceptable electrochromic properties can be deposited from a ceramic target using DC magnetron sputtering.

In order to understand the motivation for using a ceramic target in the first place, it is necessary to briefly consider some of the properties of the EC films. Four main measurements are taken to characterize the single layers: (1) the thickness, (2) the transmission and reflection characteristics 
(and therefore the optical constants), (3) the electrochromic efficiency, and (4) the amount of irreversibly incorporated charge held in the film after cycling (the blindcharge). Each of these properties is now considered briefly, and the behavior of films deposited from the pilot line and the ceramic target in the lab coater compared:

Thickness: It is well known that the optical absorption of EC films in the colored state obeys the Beer-Lambert law up to a point where the absorption saturates. Therefore the dark state coloration will ultimately be limited by the thickness of the EC film, and so it is necessary to deposit a thick enough layer to give an acceptable dark state transmission. This only really becomes important when a manufacturing operation is considered, where throughput becomes important, and film deposition rate becomes critical to achieving economical production. During the study of deposition from the ceramic target, it was found that the deposition rates for DC magnetron sputtered films were comparable if not higher than for films sputtered in the same sputtering system but from a metal target.

Transmission: The transmission of an EC device in the clear state will be an amalgamation of the optical properties of all of the individual layers making up the stack. It is possible to design a layer structure that can take advantage of the differences in refractive indices to boost the transmission of the overall stack for non-absorbing films. However any absorption in any of the layers will reduce the overall transmission in the clear state, thus reducing the dynamic range of the EC device. Highly transparent EC films were deposited from the ceramic target during this work. An example is shown in Figure A2. 1 in the Appendix.

Electrochromic Efficiency: The electrochromic efficiency $\eta$, of the film is defined as the change in optical density per unit charge inserted:

$$
\eta=\Delta O D / \Delta \rho,
$$

where the optical density is given by

$$
O D=\log \left(T(\rho) / T_{o}\right),
$$

where $T(\rho)$ is the transmission for an inserted charge density of $\rho$, and $T_{o}$ is the initial transmission. Once again, the measurements of electrochromic efficiency for the films deposited from the ceramic target compared favorably with those from the standard process.

Blindcharge: The blindcharge is measured by cycling the film in a lithium-containing electrolyte (wet cell) and then carefully measuring the current flowing into and out of the film. Invariably, the amount of charge inserted into the film will exceed the amount extracted, and this unrecovered charge is referred to as blindcharge. (It is "blind" as it causes no coloration of the film.) It was thought that the blindcharge would cause a problem later in the process, as the net amount of lithium remaining in the device after the lithiation process would be a simple sum of the amount inserted less the amount removed by satisfying the blindcharge on the first cycle.

There is clear evidence that this will lead to a device that will lose several percent in transmission after the first color-bleach cycle. It would therefore seem sensible to try to minimize the amount of blindcharge present in the film. 
Films were deposited from the ceramic target to try to achieve comparable electrochromic properties. The deposition parameters and some measured properties are given in Table A2. 1 in the Appendix.

The standard EC film microstructure has been compared with these films prepared using the ceramic target. The microstructure of the films from the standard pilot line process consists of a Z1-type film structure ${ }^{12}$ consisting of columns a few tens of nanometers in diameter separated by voids several nanometers wide. The top of the film contains surface domes with wider voids between. The films are at least partially crystalline.

The films were examined using a combination of Atomic Force Microscopy (AFM) for surface roughness and morphology, and with X-ray diffraction (XRD) to determine the degree of crystallinity. Initial results show some differences in both the surface topography and the film crystallinity.

Figure 2.1 shows 2-D and 3-D contact AFM traces of the pilot line EC films. The nodular nature of the film surface is readily observed in the $2-\mathrm{D}$ trace. Fine $(\sim 10 \mathrm{~nm})$ scale detail in the images arises from crystallite grain boundaries. Roughness $\left(R_{a}\right)$ values are on the order of $13 \mathrm{~nm}$ with a peak-to-valley distance of $230 \mathrm{~nm}$.

An example of a similar measurement from a film produced using the ceramic target is shown in Figure 2. 2. The roughness $\left(\mathrm{R}_{\mathrm{a}}\right)$ value is slightly less than is generally seen from the pilot line films, and the peak-to-valley distance $(78 \mathrm{~nm})$ appears significantly lower than that from the pilot line film.

There is a significant difference in the degree of crystallinity between films deposited from the ceramic target and those from the pilot line (Figure 2. 3). The ceramic target films are amorphous, while the pilot line films show peaks consistent with crystalline monoclinic EC films.

The films described above were then fabricated into EC devices by completing them on the pilot line, using the standard process. A device using a standard EC film was also run to provide a control sample. For the samples prepared using the ceramic target, the gas flow and substrate temperature were varied between two levels to give a simple factorial design of experiment, and then the level of lithium inserted during device fabrication was varied for each of the pilot line runs. Everything else was held constant. There were four different levels of lithium varying from the nominally standard level to a very much lower level, and these levels are labeled $0,12,25$, and 30 (25 and 30 are close to the nominal level). 
Peak Surface Area Sumit Zero Crossing Stopband Execute Cursor

Roughness Analysis
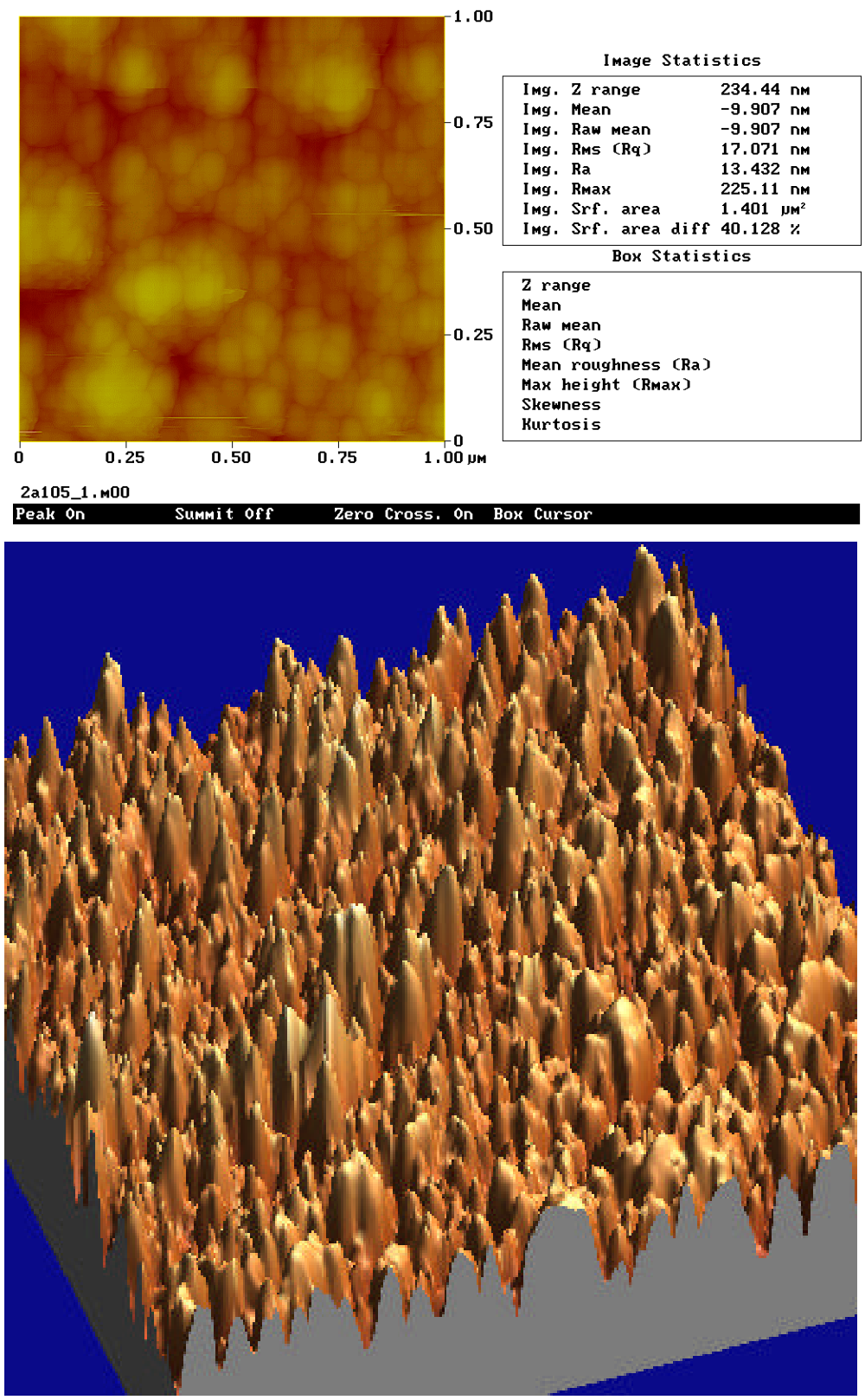

Figure 2.1 - (a) 2-dimensional and (b) 3-dimensional AFM plots of pilot line EC films. Roughness $\left(R_{a}\right)$ values are on the order of $13 \mathrm{~nm}$ with a peak-to-valley distance of $230 \mathrm{~nm}$. 
Peak Surface Area Sumit Zero Crossing Stopband Execute Cursor

Roughness Analysis
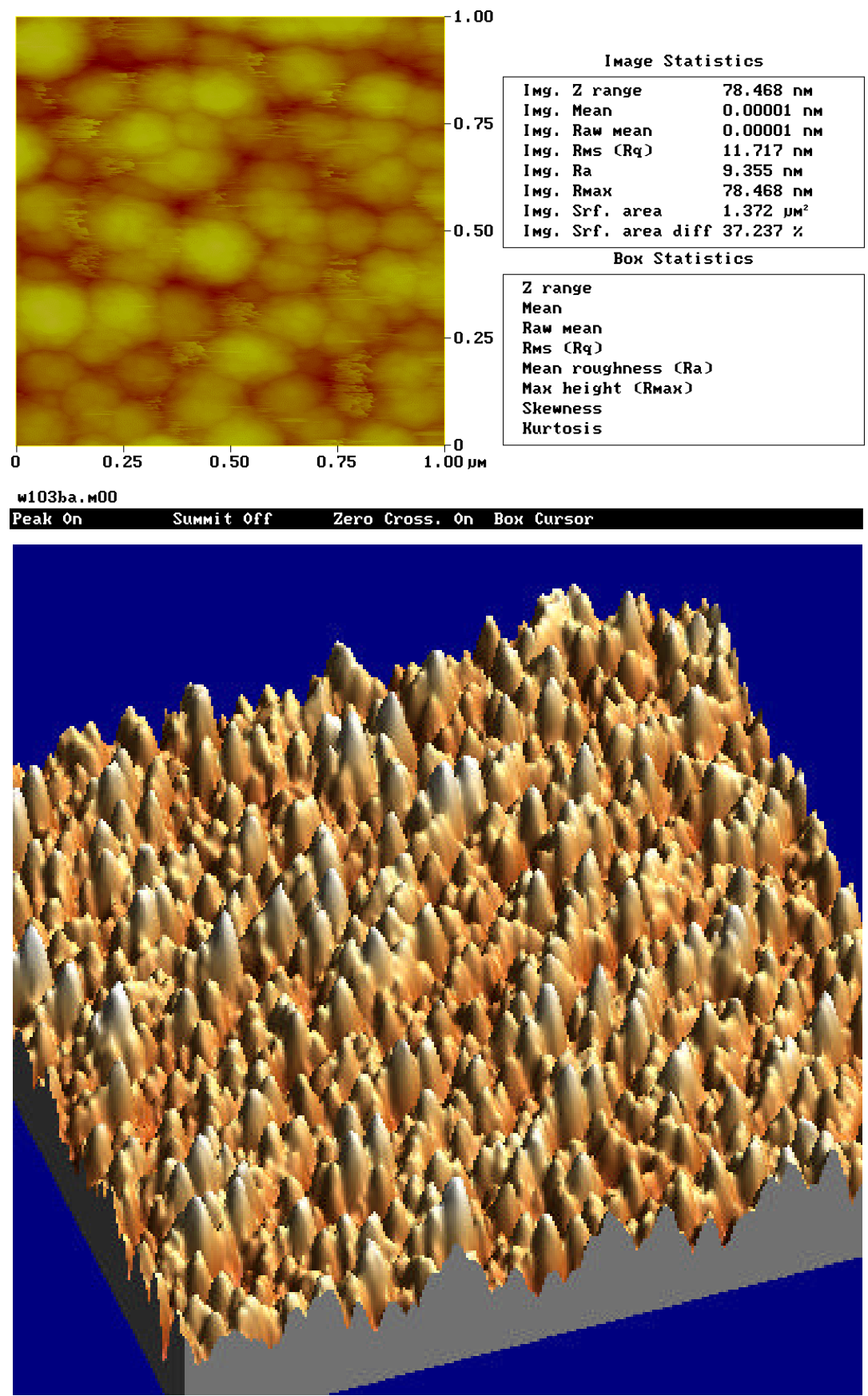

Figure 2.2 - (a) 2-dimensional and (b) 3-dimensional AFM plots of EC films produced from the ceramic target. Roughness $\left(R_{\mathrm{a}}\right)$ values $(9 \mathrm{~nm})$ are slightly lower than for the standard films, whereas the peak-to-valley distance $(80 \mathrm{~nm})$ appears significantly lower than for the pilot line film. 


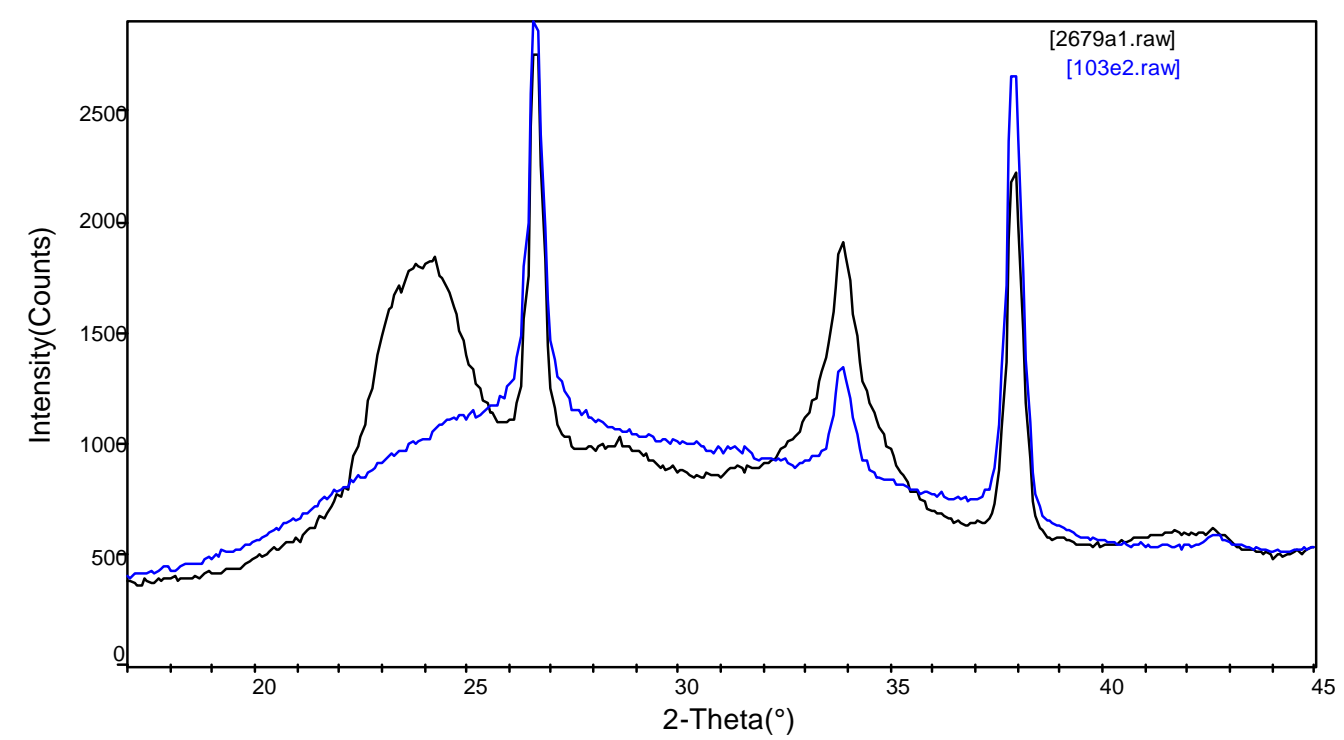

Figure 2. 3 - XRD comparison of pilot line (black) and ceramic target (blue) EC films. The ceramic target data suggests that the films are amorphous; the pilot line film pattern suggests a crystalline film. The large hump in the data from the pilot line sample centered at around 24을 -theta is a combined triplet from the monoclinic (200), (020) and (002) peaks. Crystallite size determination from diffraction pattern modeling suggests an average of 5-10 $\mathrm{nm}$. The strong diffraction peaks at $\sim 26.5,34$ and 38을 2-theta are from the underlying conductive oxide film.

In general, the following results were found for films deposited from the ceramic target:

a The devices that were lithiated at, or close to, the normal level (i.e. 25 or 30) appeared to be significantly overlithiated - i.e. they were initially blue (in the clear state) and electronically very leaky. The measured initial transmission i.e. the clear state, for these samples varied from $63 \%$ down to $43 \%$.

- The level of initial blue coloration (clear state) was the worst in devices with EC layers deposited onto the highest temperature substrates and the lowest level of oxygen in the sputtering environment. The highest transmission in the initial clear state was for the opposite preparation conditions.

- All devices showed a significant level of "blotchiness" during switching and in the colored state except for the control samples, all of which showed no blotchiness at all.

- Reducing the level of lithium in these samples dramatically increased the bleached state transmission, (up to around $70 \%$ for lithiation levels 0 and 12).

- The colored state transmission for all the samples was very poor. It was marginally better for the "normally" lithiated samples (although these are unacceptable as devices because of the low clear state transmission). Colored state transmission varied from $7.6 \%$ up to $61.5 \%$. 
口 The colored state leakage current was lower for samples lithiated to a slightly lower level than standard (i.e. 12) but this did not correspond to the darkest coloration, as was expected. However, all of the devices show significant levels of leakage compared to standard devices.

To achieve the goal of replacing the current EC film process, it will be necessary to (i) increase the dynamic range, while maintaining the high initial bleached state, (ii) reduce the leakage current, and (iii) improve the cosmetic appearance of the device. It is anticipated that this will involve further characterization of the EC layer process itself, some optimization of the pilot line process to accommodate the different EC layer characteristics, and potentially some modifications to the IC process to compensate for the changes introduced by the different EC layer properties.

Several other attempts have been made to try to produce devices with films deposited from the ceramic target, but all have produced the same result where the samples are extremely electronically leaky, while the control samples behave normally. The conclusion that there is an interaction between the IC (which is assumed to dominate the electronic behavior of the device) and the underlying EC films, is therefore inescapable. An investigation of the nature of these interactions prior to proceeding with any of the tasks associated with the ceramic target was therefore undertaken, and the results of that study described in Section §2.1.3.3.

\section{\$2.1.1.2.2 Thin film deposition with reduced gas flow}

Thermal processing is required for the current EC film deposition process. This has implications of large capital cost in the upcoming production facility, as well as causing problems in obtaining repeatable and uniform films. EC films have been produced that perform reasonably well in a wet-cell evaluation when deposited in the transition region between the oxide and metallic modes. Figure A2.2 (Appendix) shows the measured hysteresis curve for the pilot line process.

These data are obtained by running the target at a fixed power and pressure and measuring the voltage as the gas flows are varied in a controlled manner. Beginning with an oxygen rich environment, the argon level is gradually increased, and the pressure maintained constant by automatically controlling the oxygen flow. This process is continued until the target flips metallic (indicated by a sharp increase in the voltage), and then the process is reversed and the argon flow is reduced.

For this study, EC film samples were run at various points on the hysteresis curve, but with no substrate heating, and their electrochromic properties evaluated by the usual wet-cell techniques. It was found that there is residual absorption in these films if they are run at too high a voltage, (i.e. close to the metallic regime) but very acceptable properties can be obtained for films run in the transition region.

A run of four complete EC devices was made, where the only deviation from the standard process was to deposit the EC layer in the transition region. Transmission performance is summarized in Table A2.2 (Appendix).

There is a significant loss of transmission upon cycling, but this is probably due to there being a small amount of residual blindcharge, and could be addressed by increasing the amount of lithium inserted into the device. It was also noted that the devices were somewhat electronically leaky, suggesting once again that the nature of the EC layer is important in determining the electronic properties of the device. However, the devices switched fairly well, coloring to around 
$10 \%$ in each case. The variation is most likely due to an unintentional variation in the level of lithium, due to non-uniformity seen at the time of these experiments.

In summary, depositing EC films in transition mode shows promise for reducing thermal processing of the layer. The reduction of the bleached-state transmission after cycling indicates that there is still excessive blind-charge, and once again, this should be easy to rectify by adjusting the amount of lithium inserted into the device. In addition, this process variation shows the potential to cut deposition time by $75 \%$ because the deposition rate is $4 \times$ that of the standard process. This also means that the capital requirements for cathodes, power supplies, pumps and flow controllers for a production coater would be significantly reduced.

\section{\$2.1.1.2.3 Conventional process with reduced thermal processing}

As reported in the previous section, very limited success was obtained for devices fabricated with EC films deposited from a ceramic target. These devices almost always show poor coloration and high electronic leakage currents. As a result, an alternative approach was taken. This took the form of an evolution of parameters (EVOP) experiment where one factor is varied in a known manner, and the results of device measurements examined for significant differences during the initial characterization test. By varying the thermal processing time (at constant temperature) between reasonable limits, few significant differences were seen. However, devices with reduced thermal processing process show a slight increase in transmission upon cycling, while those with a longer thermal process show a slight decrease. There were also minor changes in the bleached state transmission and bleached state color. It was concluded that the thermal processing times can be reduced by almost a factor of two while maintaining satisfactory device performance.

\section{\$2.1.2 CE modifications}

The $\mathrm{CE}$ in electrochromic devices is a critical layer. It is used to store the charge, which is inturn used to color the electrochromic layer when the device is in the clear state. It must therefore have several properties: (i) It must allow charge to intercalate easily; (ii) it must be stable and durable to repeated cycling; and (iii) to maximize the clear state transmission, it must display little or no absorption in the fully intercalated state. ${ }^{13}$ SageGlass ${ }^{\circledR}$ windows use a proprietary composition that has all these properties.

The counter electrode (CE) used in the SAGE device has been shown to be very stable to repeated charge insertion/extraction cycling, but may possibly display a small amount of residual absorption when the device is fully bleached. The challenge is to remove this absorption without sacrificing the wide dynamic range and good switching kinetics of the device.

The $\mathrm{CE}$ used is a thin film of a proprietary metal oxide mixture, which will be referred to as $\mathrm{A}_{a} \mathrm{~B}_{b} \ldots N_{n} \mathrm{O}_{x}$, where $a$ is the percentage of metal A by weight, $b$ is the percentage of metal $\mathrm{B}$, etc., and $x$ is the mole fraction of oxygen. Currently the CE used is a particular composition for pilot line studies, and this is referred to as CE-I.

The work reported here will concentrate on experiments carried out with one particular target with an altered composition, labeled CE-II. 


\section{\$2.1.2.1 Variations caused by different CE compositions}

Thin films from target CE-II were deposited in the lab coater to determine sputter deposition conditions that would yield thin films with properties suitable for this application. It was found that for similar deposition parameters, a number of properties in the as-deposited films were different from those obtained from CE-I.

The sputter rate was faster for CE-II when compared to CE-I resulting in film thickness approximately $30 \%$ greater than for the "normal" CE (CE-I). Naturally, this will only be significant if the thickness requirements for the $\mathrm{CE}$ remain the same for the different composition, but an increase in deposition rate is obviously beneficial when moving into a production setting, as it will potentially reduce capital outlay and/or cycle time.

The film deposition described above yields a transmission (as-deposited) for the CE-II film which is $\sim 25 \%$ greater than for CE-I, indicative of the changed composition.

Work carried out to understand the optical properties that can be obtained from the two different $\mathrm{CE}$ compositions has focused on depositing $\mathrm{CE} / \mathrm{Li} / \mathrm{TC}$ stacks on witness slides, and studying the optical properties of these, without the complications of the underlying TC/EC layer/IC layers. In addition, some $\mathrm{CE}$ films have been deposited onto glass coated with the TC to study the electrochemistry to ensure that this is still adequate for the purpose.

Samples of CE-I/Li/TC were deposited onto clear glass slides and the optical properties determined. Some samples were heated, and the optical properties re-measured. Some typical data are given in Table A2.3 in the Appendix. It is noted that the transmission and color coordinates for this partial stack are close to those obtained for the whole device, so it is reasonable to assume that any improvements in optical properties made on this partial stack will be transferred over to the whole device. The focus is placed on $b^{*}$ as the parameter needed to be controlled. ${ }^{14}$ In general a range of $b^{*}$ values for these stacks is found, and this is reflected in the values measured in finished devices.

The aim is to increase the visible light transmission, $(\mathrm{T}(\%))$ and reduce the value of $b^{*}$ to somewhere close to zero without compromising the charge capacity or electrochemistry.

Samples of CE/Li/ITO were deposited in an identical way, but this time using the CE-II target. Some values for the same measured quantities are given in Table A2.4 in the Appendix. 
It is clear that $b^{*}$ is lower for the majority of these films than the values seen for equivalent CE-I stacks. It is therefore assumed that using the new CE composition will lead to a reduction in $b^{*}$ for the devices.

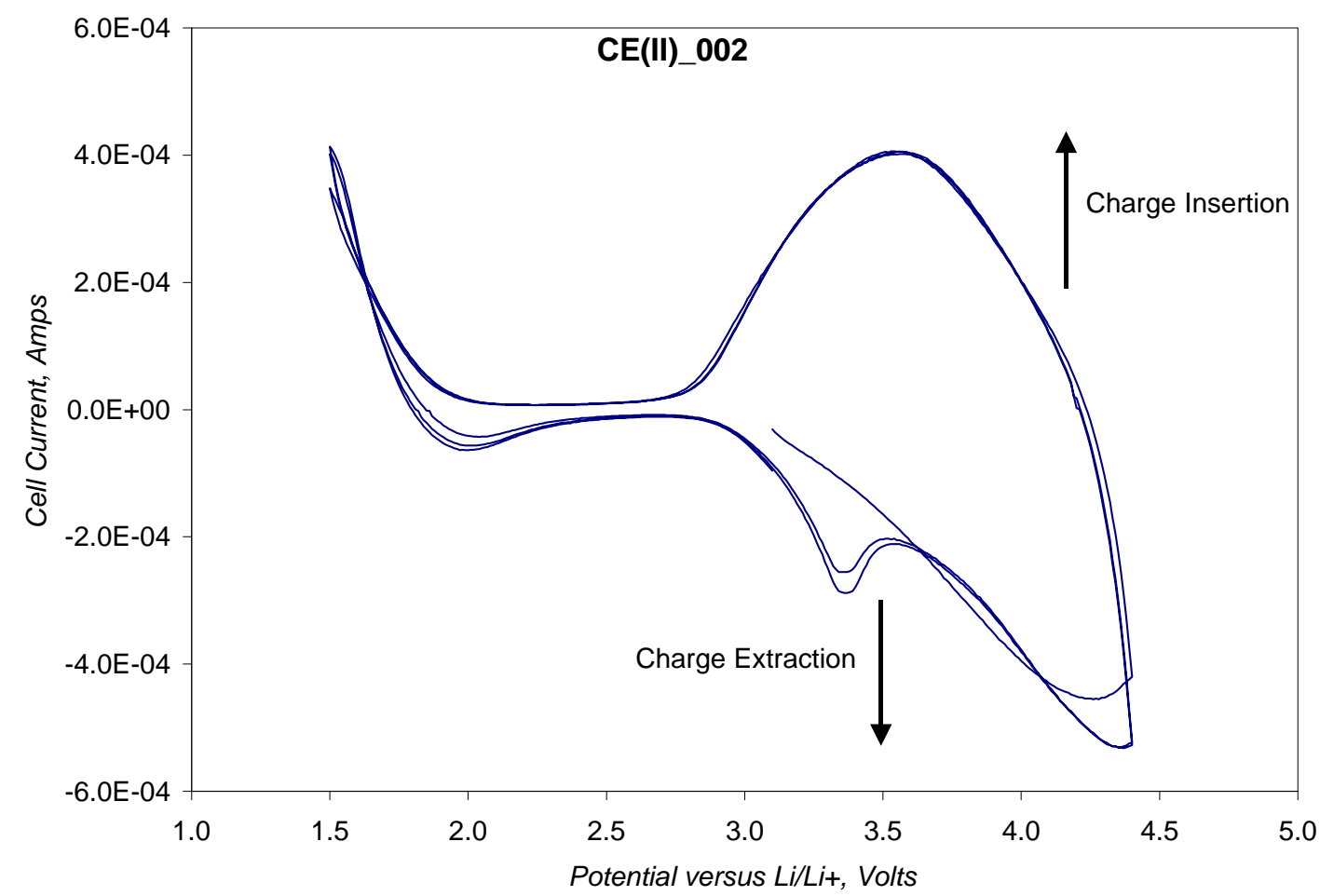

Figure 2. 4 - Electrochemical measurements of a CV of a sample of CE-II in a lithium containing electrolyte. Sweep rate was $20 \mathrm{mV} / \mathrm{s}$ and the sample area was $4 \mathrm{~cm}^{2}$. The open circuit potential was approximately $3.1 \mathrm{~V}$ vs. $\mathrm{Li} / \mathrm{Li}^{+}$.

The electrochemistry of CE-II films looks very well behaved and reversible. A cyclic voltammogram (CV) of lithiated CE-II is shown in Figure 2. 4. (For details on measurement and interpretation of CV's, the reader is referred to A.J.Bard and L.R.Faulkner 'Electrochemical Methods', Pub: Wiley (1980), Chapter $6^{15}$ ). In this measurement the voltage between the thin film of interest and a reference electrode (in this case lithium metal) immersed in the electrolyte is measured, and recorded. This is the open circuit voltage, $V_{\text {occ }}$ The voltage then is swept at a controlled rate (in this case $20 \mathrm{mV} / \mathrm{s}$ ) from $V_{\text {oc }}$ to some predefined anodic and cathodic limits and the current recorded. A flow of current represents an electrochemical reaction between the electrode and the species in the electrolyte, in this case lithium insertion and extraction. The transmission of the film using a diode laser and photodetector is measured at the same time as the $\mathrm{CV}$ so that the optical changes can be related to the electrochemistry.

Features in a CV can be interpreted in terms of the electrochemical processes which are occurring and can lead to a better understanding of the electrochemistry of the CE films, and hence the overall behavior of the device.

The example in Figure 2. 4 shows that there is a clear separation between different lithium insertion reactions. Beginning at approximately $3.0 \mathrm{~V}$ vs $\mathrm{Li}$, the voltage is first swept to higher potential, i.e. $4.5 \mathrm{~V}$ vs $\mathrm{Li}$, and a (negative) current flows out of the electrode. This is indicative of 
electrons flowing out of the electrode into the external circuit, and positively charged ions being ejected into the electrolyte. The voltage is now swept back to a less positive value, and charge is re-inserted into the $\mathrm{CE}$ film. As the voltage is swept further, the current reaches a limiting value, (dependent upon the sweep rate and the charge capacity of the film), and then reduces back towards zero. A second charge insertion peak can be seen at voltages below $2 \mathrm{~V}$ vs Li. As the sweep direction is once again reversed, this cycle is repeated.

Given the behavior seen here, there is a high level of confidence that films deposited from the CE-II composition target will show good electrochemical behavior when used in an EC device.

\section{\$2.1.2.2 Impact of gaseous dopants on structure and interface characteristics of $\mathrm{CE}$}

The absorption of the CE film is an important indication of the state of oxidation of this layerhigher absorption indicates a more oxidized film. It is important to maintain consistency of the oxidation state of this film to maintain consistency of the performance of the final product, as this is likely to affect the optical properties and the electrochemical behavior of the layer.

Single layers deposited on clear glass on the pilot line coater have shown variation in the thickness and absorptivity which appear to be related to the background contamination (base pressure) in the sputtering machine. This is shown in Figure 2. 5, where the value of $k$ at 500nm ${ }^{\S}$ is taken as a measure of the absorption for these films. These films were all deposited under nominally identical conditions during a relatively short period of time. There is a clear spread in these data, both for the absorption and the thickness, hence this study to determine which contaminant gas or gases are responsible.

To investigate this, a study in the lab was undertaken. Single layers of CE were deposited onto $7.6 \mathrm{~cm} \times 2.5 \mathrm{~cm}(3 " \times 1 ")$ glass slides under various well-controlled conditions. The transmission and reflection spectra of these layers were then recorded, and from these data, the thickness and optical constants determined. Different gas compositions were used to determine the effects of small amounts of background impurities on the properties of the CE films.

\footnotetext{
${ }^{\S} k$ is determined by measuring both the reflection and transmission of the film, and then fitting an optical model to
} these data. The variables in the fit are the optical constant ( $n$ and $k$ ) and the thickness. 


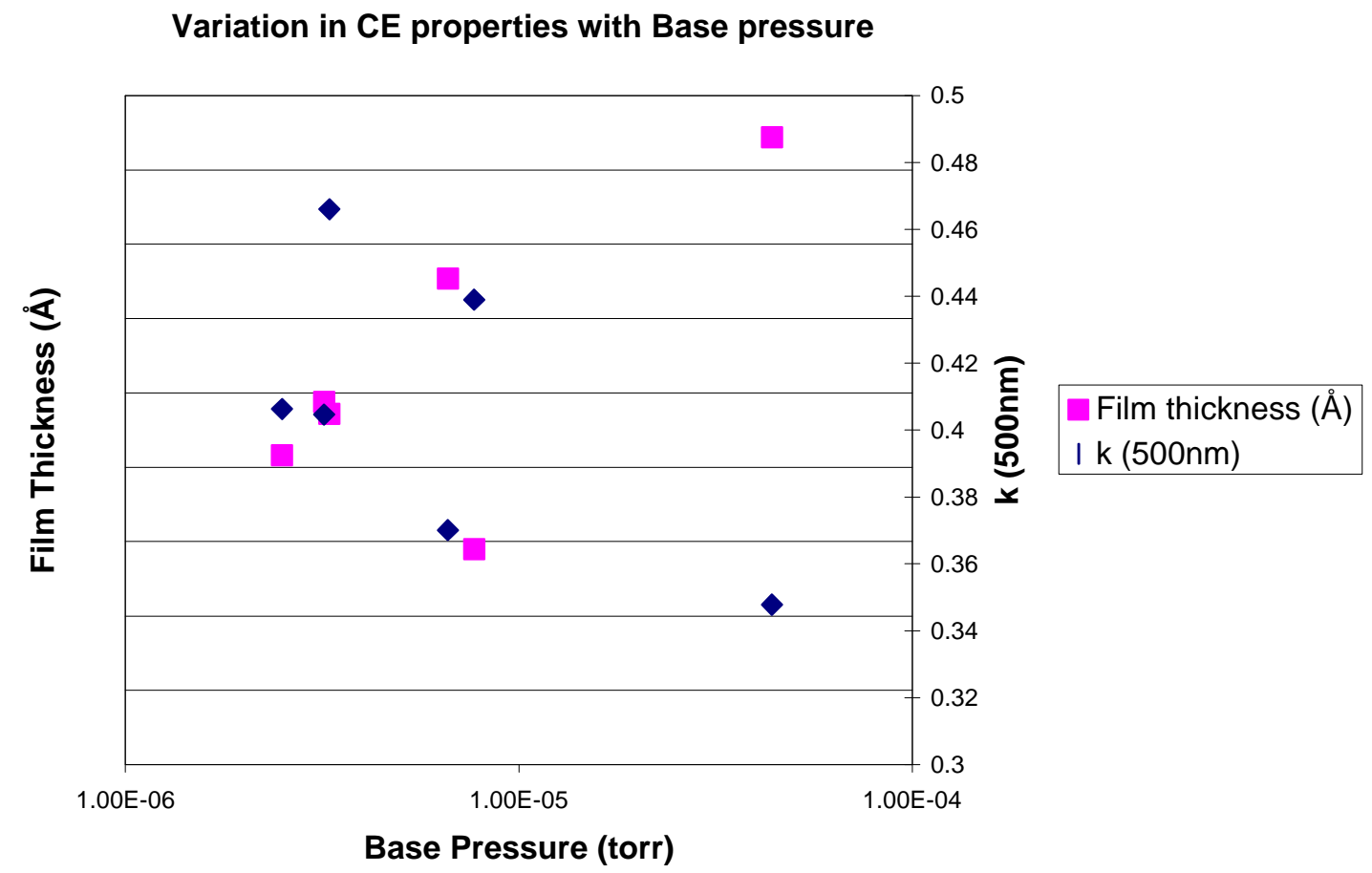

Figure 2. 5 - Variation in CE film properties shown as a function of base pressure for films deposited in the pilot line sputtering machine. All films deposited under the same conditions.

The nitrogen, $\mathrm{CO}_{2}$ and argon additions to the oxygen-sputtering environment were simply obtained by using a separate gas bottle and flow controller. The water was added by bubbling oxygen through water - held at or close to $0^{\circ} \mathrm{C}$ - and then diluting this with dry oxygen to obtain a variety of different water levels. The bubbler was fitted with a diffuser to ensure the highest contact of gas with water.

Each film was deposited after allowing the chamber to pump down for at least 30 minutes; generally this meant that the chamber pressure was less than $3 \times 10^{-6}$ torr, and the target was presputtered for 30 minutes prior to film deposition.

The nominal conditions for the CE deposition are proprietary. Several films were deposited under standard conditions from time to time throughout the study as a check. The repeatability of the results from this series shows that there is no long-term drift of the process.

\section{Effect of Argon}

Argon was added to the standard sputtering gas and some oxygen was removed to maintain approximately the same pressure. The deposition conditions were therefore slightly modified from the standard conditions. The number of passes was reduced to maintain the thickness close to a target thickness when it became apparent that adding Ar increased the deposition rate.

The results for a number of different depositions are shown below in Figure 2. 6, giving some idea of the repeatability of this measurement. Clearly, there is little or no effect on the absorption of these films, but the deposition rate is considerably enhanced by addition of argon. Note that 
several of the films deposited with Ar were made with double the number of passes, but there is no significant difference seen-except the obvious thickness difference- the thickness deposited per pass remains the same. Note the spread of data for the absorption is very similar both with and without argon, indicating no effect of argon on the film properties at this level.

\section{Effect of adding Argon to sputter gas for CE deposition}

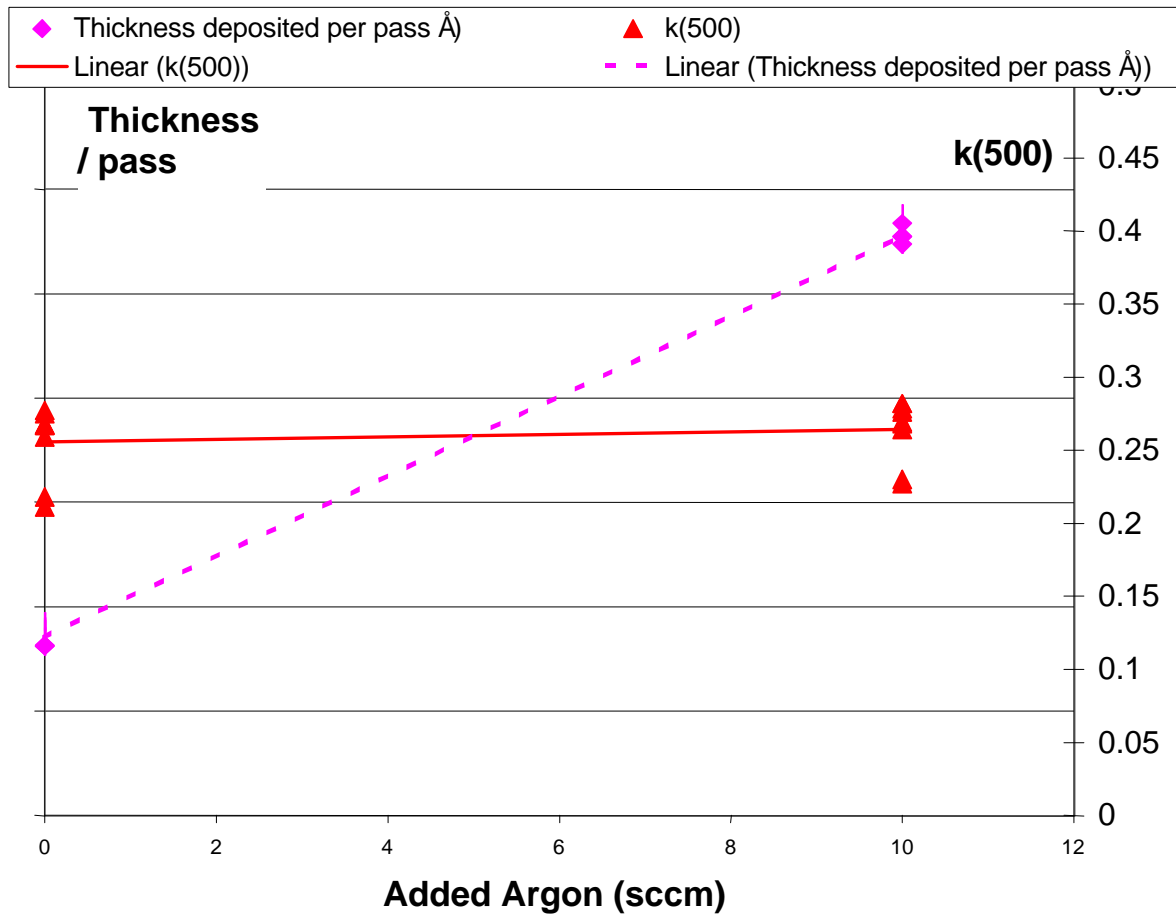

Figure 2. 6 - Effect of argon addition on the deposition rate and optical absorption of CE films deposited in the lab coater. Conditions for the films deposited with argon added are given in the text.

A useful increase (a factor of two) in deposition rate was achieved by the addition of small amounts of argon, with no apparent change in the optical properties of the films. It is likely that further increases in deposition rate are possible by further argon addition. This will be useful in two ways: (i) The requirements for cathodes/deposition zone in the production coater will be reduced; and (ii) because the film is deposited faster, there will be less deleterious impact of the effective concentration of background residuals incident on the growing film.

\section{Effect of water addition}

Water was added as described above, by bubbling oxygen through ice water in a bubbler held in a water bath at zero ${ }^{\circ} \mathrm{C}$. Using this arrangement, fully saturated oxygen containing approximately $6000 \mathrm{ppm}$ by volume (or 3640ppm by wt) were produced. 
The results are shown in Figure 2. 7, where the data are plotted as a function of wet oxygen flow - $60 \mathrm{sccm}$ represents $100 \%$ wet oxygen flow, and are therefore as saturated as possible. Very little effect is seen on either the thickness or the absorption, although there may be a slight decrease in absorption (results are plotted as the reciprocal of $k(500 \mathrm{~nm})$ ) with increase in the level of water. It is assumed, however, that the residual water levels in the pilot line will not reach the extremely high levels seen here.

\section{Water Dependence of CE properties}

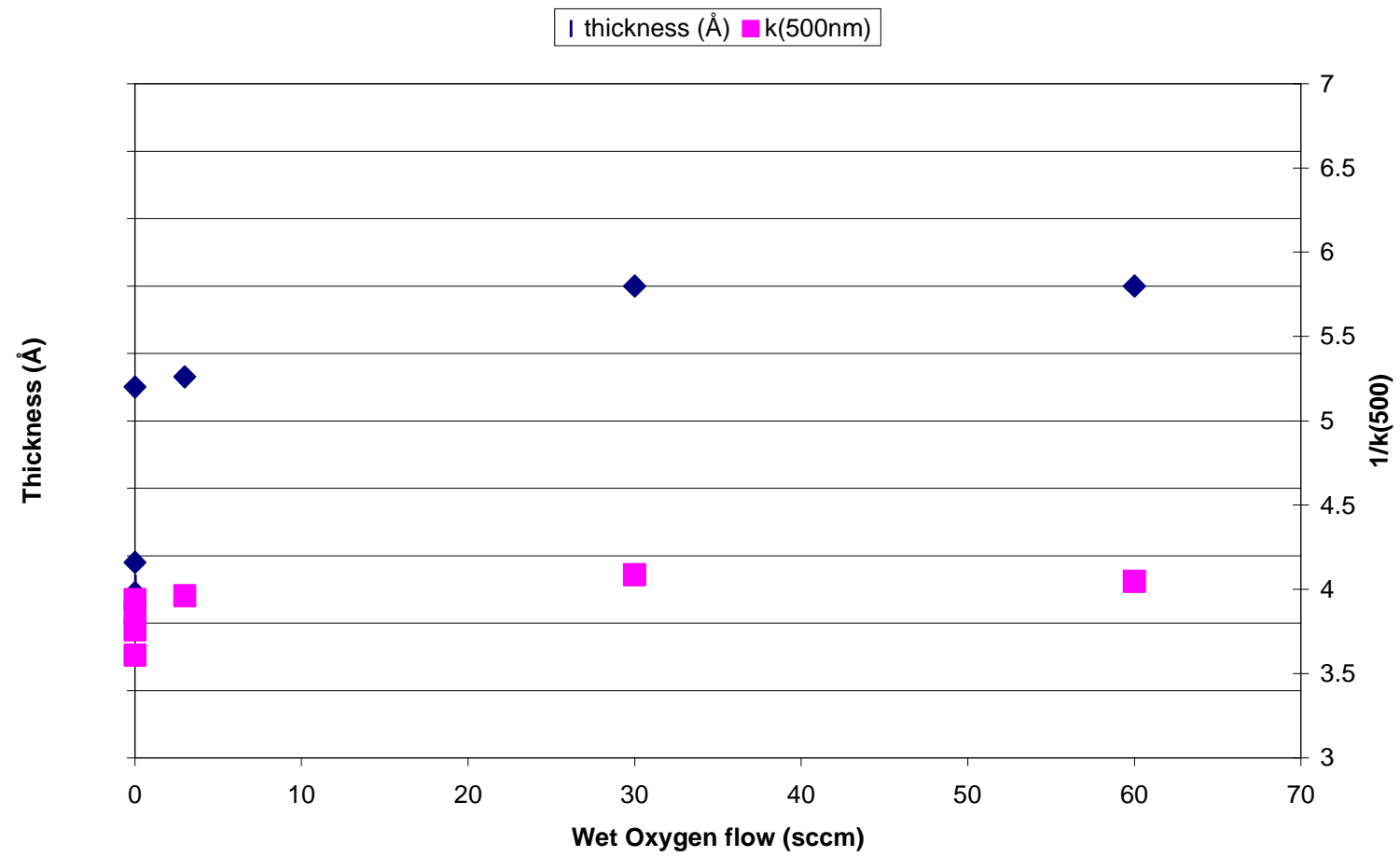

Figure 2. 7 - Effect of water addition on the deposition rate and optical absorption of CE films deposited in the lab coater. Conditions for the films deposited with water added are given in the text. A wet oxygen flow of $60 \mathrm{sccm}$ corresponds to approximately 6000ppm.

\section{Effect of Nitrogen}

Nitrogen was added to the sputter gas and the results of the thickness measurements are given in Figure 2. 8. Other data points are shown for samples that were deposited (a) at slightly higher temperatures (around $150^{\circ} \mathrm{C}$ ) and (b) some with an addition of $\mathrm{CO}_{2}$. All of the measurements show little or no dependence of the thickness on any of these parameters. Very good consistency of thickness reproducibility is observed. 
Thickness dependence on Nitrogen flow

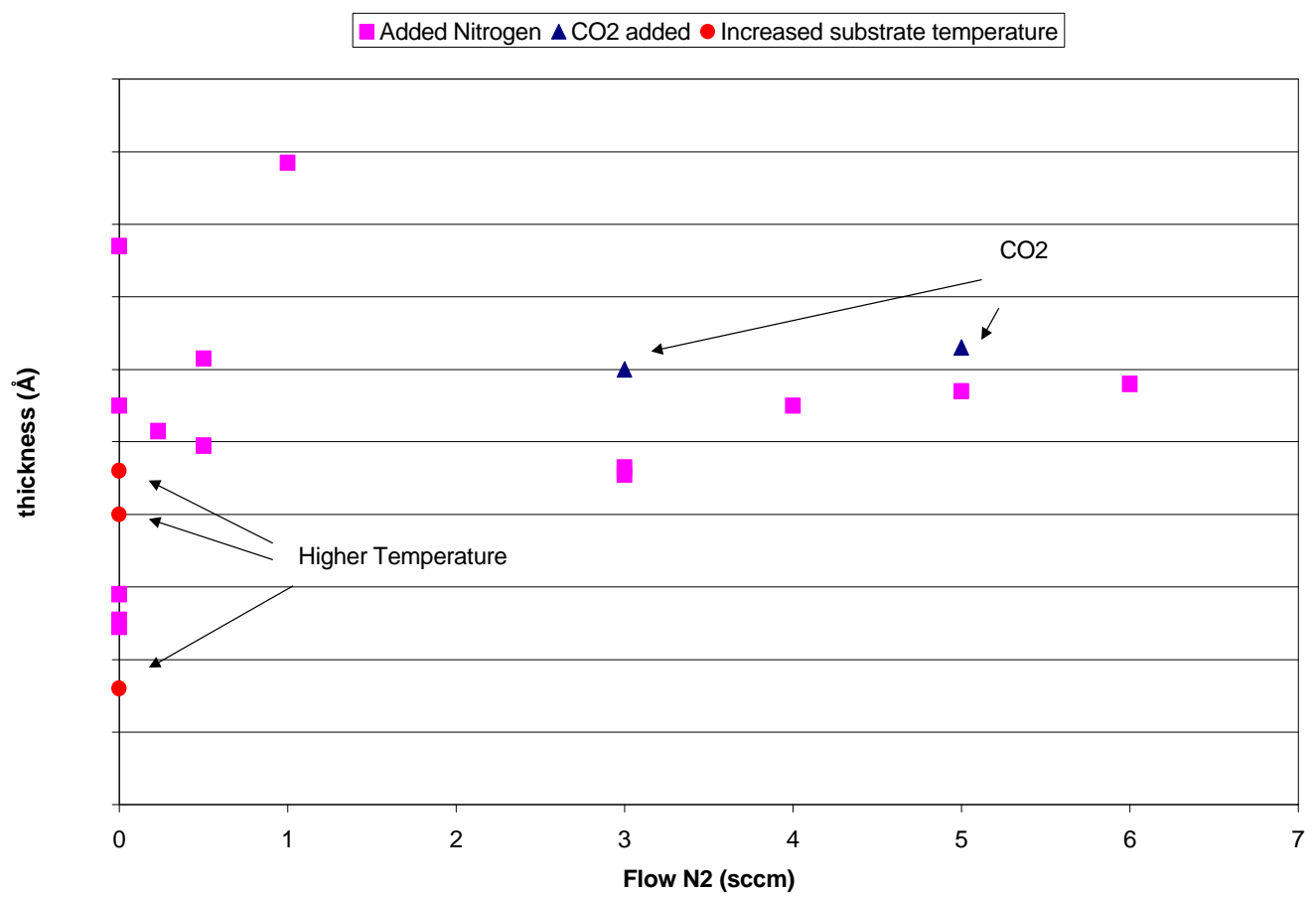

Figure 2. 8 - The thickness dependence of films grown with different levels of nitrogen contamination. In addition, several films deposited at elevated temperature (red circles) and two films deposited with $\mathrm{CO}_{2}$ addition (blue triangles) are also shown. Note there is no dependence of thickness on either $\mathrm{N}_{2}, \mathrm{CO}_{2}$ or heating.

\section{Effect of Nitrogen on absorption of CE}

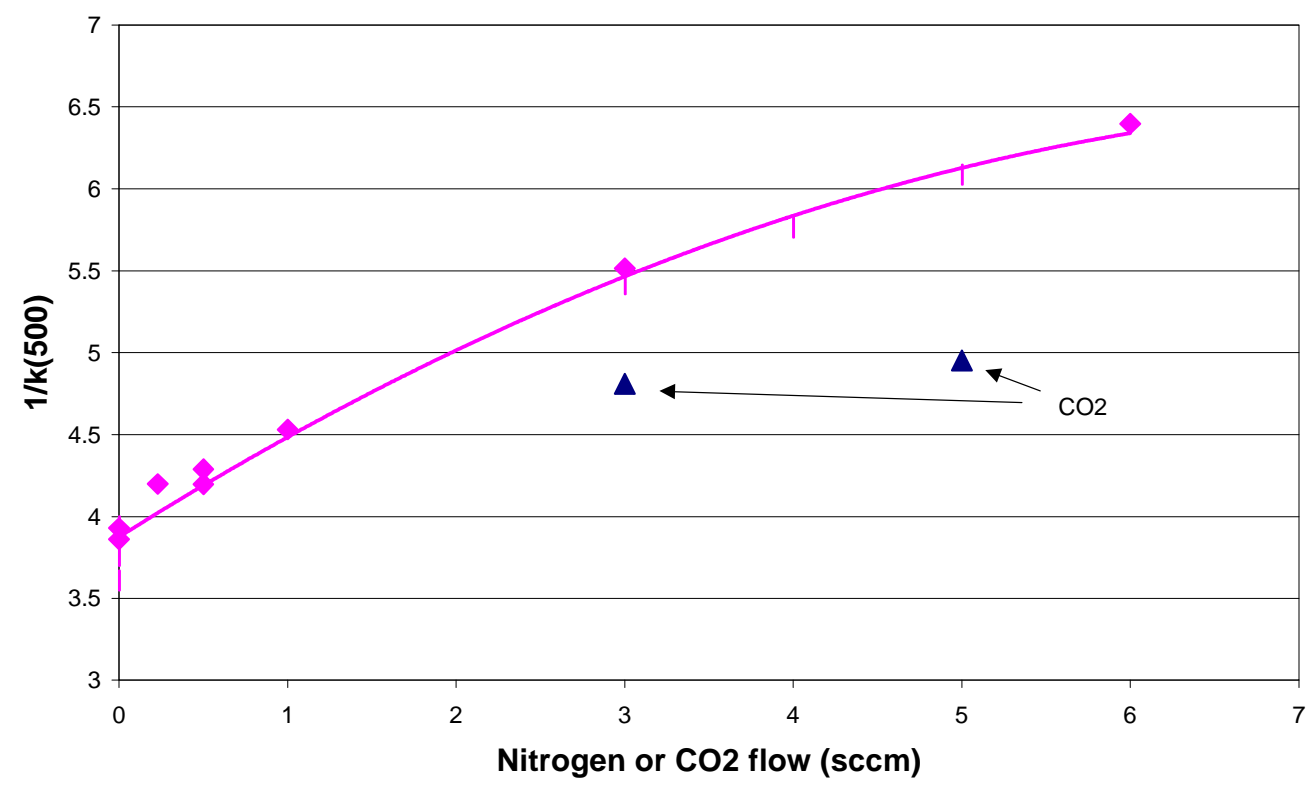

Figure 2. 9 - Effect of adding nitrogen to the sputtering gas on the measured absorption for a number of thin films. The values for $\mathrm{CO}_{2}$ are also shown for comparison. 
Figure 2. 9 shows the measured absorption for the same films as a function of nitrogen addition, and also for the films with added $\mathrm{CO}_{2}$. An increase in the value of $1 / k$ (the reciprocal of absorption shows the same trend as the transmission) is seen; hence an increase in the level of nitrogen produces an increase in the transmission of the film. (A similar trend is seen for the $\mathrm{CO}_{2}$, but this is less of an effect than for the nitrogen.)

Increases in $1 / \mathrm{k}$ from around 4 to around 6 represent a transmission change from $\sim 33 \%$ to $\sim 48 \%$, which is a significant difference.

It is clear that a small air leak into the coater could have a significant effect on the properties of the $\mathrm{CE}$ layer. This is most likely to be a result of the nitrogen rather than the $\mathrm{CO}_{2}$ because of the higher percentage of nitrogen in the air compared with $\mathrm{CO}_{2}$.

Effect of $\mathrm{CO}_{2}$

As seen above, the effect of $\mathrm{CO}_{2}$ seems to decrease the absorption, but has little effect on the

\begin{tabular}{|c|c|c|}
\hline Contaminant & Absorption & Thickness \\
\hline Argon & No effect & Increases \\
\hline Nitrogen & Decreases & No effect \\
\hline Water & No effect & No effect \\
\hline $\mathrm{CO}_{2}$ & Decreases & No effect \\
\hline Temperature & No effect & No effect \\
\hline
\end{tabular}

Table 2. 1 - Effects of contaminants on CE Properties thickness. The magnitude of the absorption change seems to be about a factor of two less than that from nitrogen.

The optical properties of the CE have been found to be sensitive to nitrogen and $\mathrm{CO}_{2}$ and insensitive to water and low level heating. This is summarized in Table 2. 1 .

So far, nothing except Ar has been found which would give rise to the increase in thickness with increasing base pressure seen from the data given in Figure 2. 5, but residual levels of nitrogen from the air can easily explain the differences seen in optical properties.

\section{\$2.1.2.3 Modifications to the CE sputtering process}

Variations in the optical properties of the CE continued to be an issue. Figure 2. 10 shows the measured transmission for witness pieces deposited onto glass slides in the pilot line coater. The six witness pieces from each run are spaced evenly across the width of the cathode. Each "string" of joined points represents measurements of transmission taken from one run, and in most cases there are six points. The point on the left of the string represents the console side sample, and that on the right represents the pump side. Any variation in a string indicates a "side-to-side" variation. All of the films discussed here are deposited under nominally identical conditions, where the power, pressure, gas composition, line-speed and number of passes*** are all held constant. The pressure is held constant by continually adjusting the gas flow into the slot using a closed loop feedback. The data here represent witness piece runs carried out since between March and November 2002.

\footnotetext{
** In some instances the number of passes are altered but this is noted and shown in Figure 2. 10 and the data corrected accordingly.
} 


\section{Transmission of CE witness pieces}

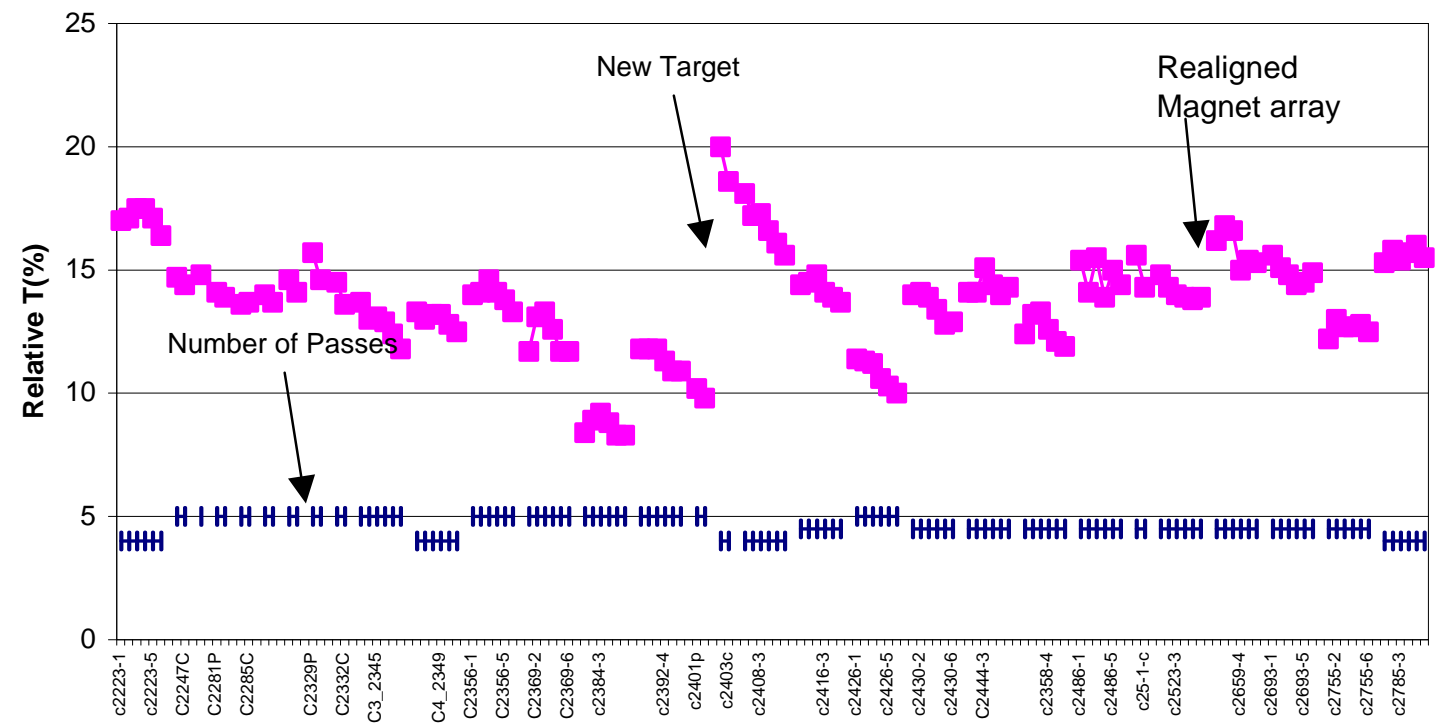

Figure 2. 10 - The variation of transmission (relative) as a function of run number showing a significant process variation. The number of passes is also indicated, showing that there is some correlation, but this is insufficient to explain the variation seen here. Each "string" of joined points represents one run, in most cases there are six points.

A clear variation in the transmission as a function of time is seen. Furthermore, during the early runs, a definite side-to-side variation in the optical properties of the $\mathrm{CE}$ is also apparent, i.e. in a direction parallel to the target. This is seen as a hump in each data string prior to the point at which a new target was installed. Following the installation of the new target, the shape of each string changed to be more indicative of a gradient from one side to the other.

Consider first the variation within a run, i.e. the side-to-side variation. Several possible causes for the variation within a run have been considered. Prior to installing the new target, there was a question as to whether the tiles of the target were bonded sufficiently to the backing plate. Indeed any voids in the bonding would likely lead to a different deposition rate from that particular tile. This was seen to be the case when the target was removed, and hence can now be eliminated as a problem. Extreme care is now taken by the manufacturer to ensure that the individual target tiles are of equal density and also bonded adequately to the backing plate.

The side-to-side variation seen in the strings of six points is clearly visible even after the target was changed, but does not seem to be consistent run to run. As this is an absorbing film, both the thickness and the absorption will determine the transmission. The optical constants and thickness are derived from transmission and reflection measurements, and it is found that while there is some variation in both parameters, of most concern is the variation in the absorption, as this is indicative of a variation in the physical properties of the film itself. It is possible to obtain such a variation in at least two ways: (i) the oxidation state of the film may increase, leading to a more highly absorbing film; or (ii), the ratio of the constituent materials may vary. Both possibilities 
have been investigated, and in collaboration with the target manufacturer, have made a thorough investigation of the target stoichiometry and density.

The composition of deposited CE films was investigated, as there was some question as to whether the molar ratio of the constituent metals was varying across the target, resulting in the non-uniformity. The standard sample preparation technique for atomic absorption analysis had to be modified since one of the sample constituents was not completely dissolved in the analyte solution. A new technique was developed to ensure that complete dissolution of the components in the films occurred, and subsequent results from atomic absorption analysis of witness piece samples showed that there was no appreciable variation of composition across the target.

Adjustments to the magnetic array in the CE cathode were made. This seemed to have resulted in a fairly marked improvement in thickness uniformity across the target, from something on the order of $5 \%$ before the change, down to a variation of less than $2 \%$. This is shown in Figure 2. 10 .

\section{Device Transmission after CE and Residual Pressure vs time}

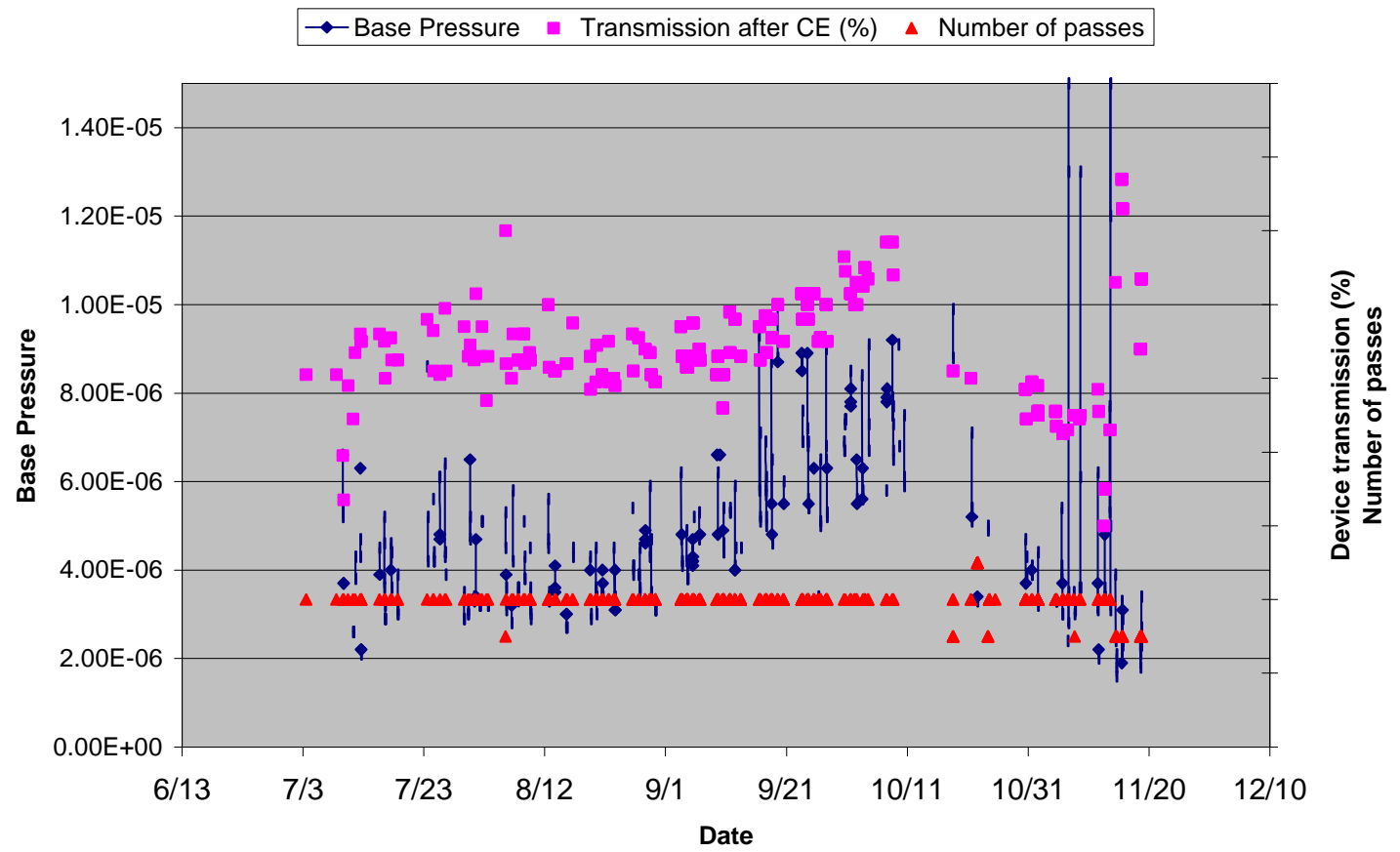

Figure 2. 11 - The base pressure of the coater prior to CE deposition as a function of time, plotted alongside the measured device transmission after CE deposition. The number of passes across the target is also shown as this will alter the thickness of the CE film, and hence the measured transmission.

The original run-to-run non-repeatability for the $\mathrm{CE}$ deposition has proven to be more troublesome to track down. The level of background impurities had a significant effect on the properties of the deposited film, and this can be seen in Figure 2. 11, where both the base pressure prior to deposition is recorded, along with the measured device transmission after CE deposition. Clearly, these measurements have the same trend with time, but this is an insufficient condition to assert that this is causal. However, the similarity is compelling, and so attempts to 
determine the origin of the base pressure variation in order to remove this variable from the process were made.

One factor that will have a significant impact on the base pressure of the system is the temperature of the load-lock following the heating processes carried out in it. A series of observations linked the transmission of the device after the $\mathrm{CE}$ was deposited to the temperature of the glass in the load-lock. It was found that the device run immediately after an EC layer run gave a significantly higher transmission than any subsequent devices. This was determined to be due to the fact that the load-lock was still hot, and caused the glass loaded for the CE deposition to be heated slightly, and it is this slightly increased temperature that leads to an increase in the transmission of the device. Some historical data indicating this effect are shown in Figure 2. 12.

It is clear that the small variations in substrate temperature that were measured were sufficient to

\section{Transmission after CE as a function of initial substrate temperature}

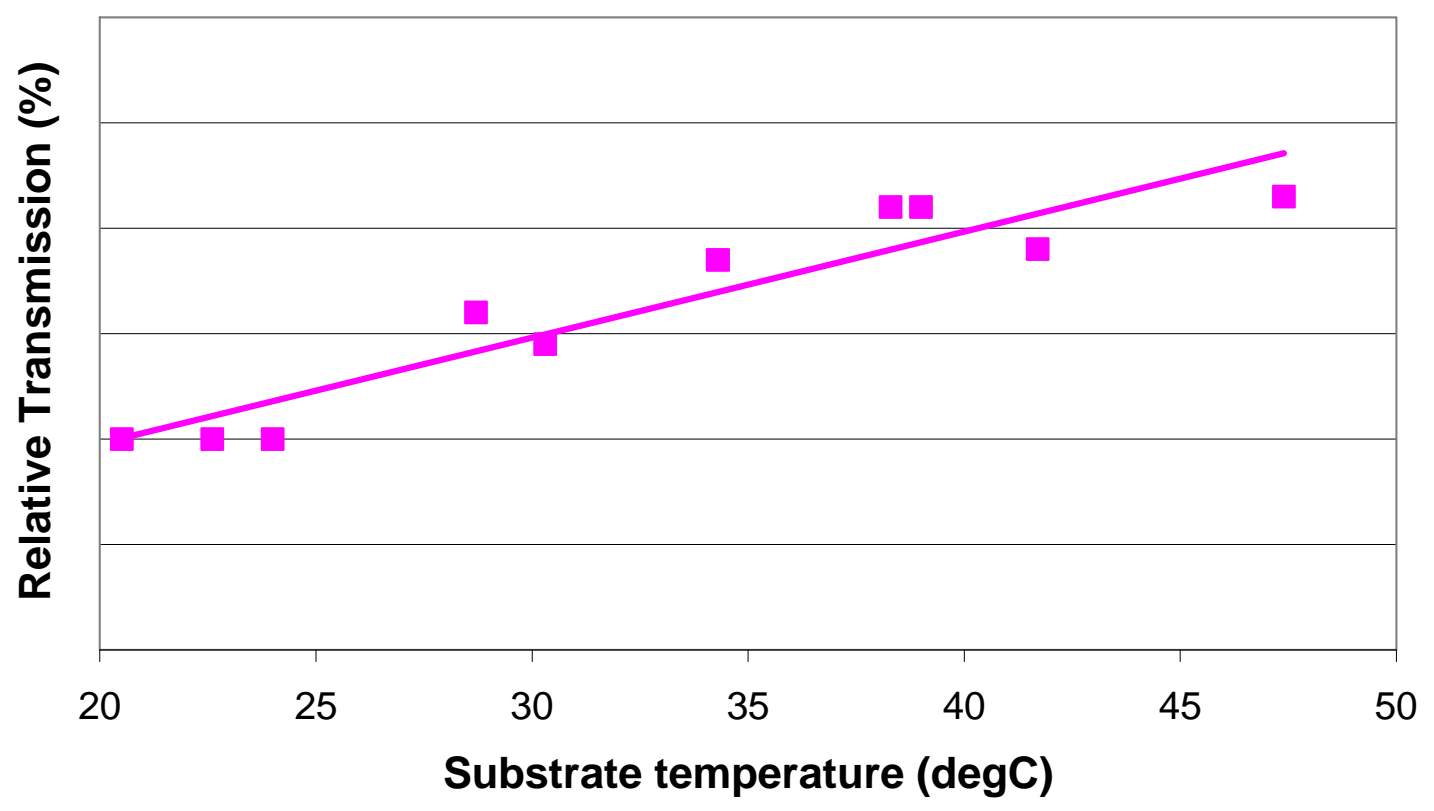

Figure 2. 12 - Transmission of devices measured after CE deposition as a function of initial substrate temperature.

cause a significant amount of the run-to-run variation that was seen. In addition, this also explains the apparent dependence of the transmission with system base pressure, because an increase in temperature in the load-lock will cause there to be more outgassing of the chamber, thereby leading to a higher base pressure.

As a result of these discoveries, the $\mathrm{CE}$ process is now run so that the substrate is heated to a consistent, but reasonably low temperature of $<90^{\circ} \mathrm{C}$, in order to eliminate as much variation as possible. By this means, the run-to-run variation has been reduced from around $6.3 \%$ to $1.3 \%$. A means for heating the glass prior to $\mathrm{CE}$ deposition has therefore been specified for the first production plant that is currently being designed. 
In conclusion, significant progress in understanding and controlling the process parameters that affect the $\mathrm{CE}$ deposition has been made. The process limits have been considerable tightened as a direct result of the work on background contamination of the CE. In addition, significant changes to the cathode have been made to improve the uniformity across the target for around 5\% at the beginning of the project, to below $2 \%$ currently. Run-to-run repeatability has been improved by attention to the details of the process, such as base pressure and temperature.

\section{\$2.1.3 Second Generation Ion Conductor}

The ion conductor (IC) layer in the SageGlass ${ }^{\circledR}$ EC device uses a patented proprietary technology which gives excellent optical, ionic and electronic properties. However, by altering the structure and composition of the IC the refractive index and conductivity can be fine-tuned, thereby maximizing the transmission range, uniformity and switching rate of the EC device. Details of the IC materials and characterization are proprietary and included in the Appendix.

\section{\$2.1.3.1 IC Materials Characterization}

See Appendix.

\section{§2.1.3.2 IC Composition and structure optimization}

See Appendix.

\section{\$2.1.3.3 Deposition of IC films}

In practice, the thin IC film does not entirely block electrons but sustains an electronic leakage current. The leakage current degrades performance by (a) increasing power consumption, (b) limiting the depth of coloration, (c) causing self-bleaching on power loss, and (d) leading to coloration gradients during switching due to Ohmic voltage drops across the TCOs.

Figure 2. 13 shows a typical electronic leakage I-V characteristic for an EC device plotted as a function of internal voltage ${ }^{\ddagger}$. Also shown is the variation of the optical density (OD) or depth of coloration with internal voltage. The OD is defined as $\log \left(\mathrm{T}_{\mathrm{ble}} / \mathrm{T}_{\mathrm{col}}\right)$ where $\mathrm{T}$ is transmission. Clearly, the I-V characteristic shown in Figure 2. 13 does not follow a simple linear relationship. There is a rapid increase in the current above a 'threshold voltage,' which is defined as shown in Figure 2. 13. Above this threshold the device is said to be 'broken-down', and the current flowing is then purely electronic leakage. For a given device structure, the threshold voltage will determine the maximum obtainable OD, and hence the dynamic range.

The leakage current can be split into two contributions: (i) that associated with the thin film stack itself (intrinsic), and (ii) that associated with localized point defects, (extrinsic). As will be discussed later (Section \$2.1.4), work carried out during this project suggested that this behavior was associated with the EC/CE interface as a result of a heterojunction. The role of the IC layer in this model is directly analogous to that of the oxide layer in a semiconductor MOS structure, ${ }^{16,17}$ but additionally serves to stabilize the junction to subsequent thermal processing. It is suspected that the forward and reverse barrier heights for the EC Layer/CE junction can be

\footnotetext{
* Internal voltage is the applied voltage minus the voltage dropped across the device series resistance and is the voltage applied directly to the EC stack.
} 
maximized by altering the composition, structure and interface chemistry of the IC. In short, the available evidence suggests that leakage current can be reduced to negligible levels through selection of appropriate process and materials for the IC layer.

The goal is therefore to develop a deposition technique for an improved IC that is compatible with high throughput production.

Replacement of the IC layer will require that the integrity of the electron barrier structure be maintained, with adequate ionic conductivity.

In practice, higher ionic conductivity typically requires a more porous, amorphous structure, and possible incorporation of lithium, both of which may degrade the electron barrier functionality. ${ }^{18}$ This calls for the discovery of a suitable materials stack, and excellent control over the respective deposition processes. Furthermore, the optical indices of the materials need to be matched to the surrounding layers, and the deposition rates need to be maintained high enough for economical processing.

The work on PVD (Physical Vapor deposition) IC has progressed along several different paths. First, optimization of the current process has continued with a view to understanding the dependence of the final device properties on deposition conditions, particularly of the IC itself, but also of several of the other critical layers. This work not only encompasses the initial testing, but also device testing carried out after cycling the devices several hundreds to thousands of times. This allows determination of how the EC devices with PVD IC will perform in a real environment.

Further, the deposition conditions of the IC are being studied in an attempt to understand the dependence of the microstructure of the IC layer - which will depend strongly on the deposition conditions - on the performance of the device as a whole. This will be evident as a difference in colored state transmission, leakage current and switching speed.

Finally, alternative materials for the IC are being considered. This is being done by depositing all of the layers except the IC on the Pilot line, while depositing the IC in the laboratory MRC coater, where changes of materials were much simpler and cheaper.

Several experimental devices have been fabricated with PVD IC layers. The simplest of these consist of a single layer, and several experiments have been done to try to optimize the deposition conditions of this layer to give a performance equivalent to that seen with the current process. 


\section{Example of color saturation at threshold voltage}

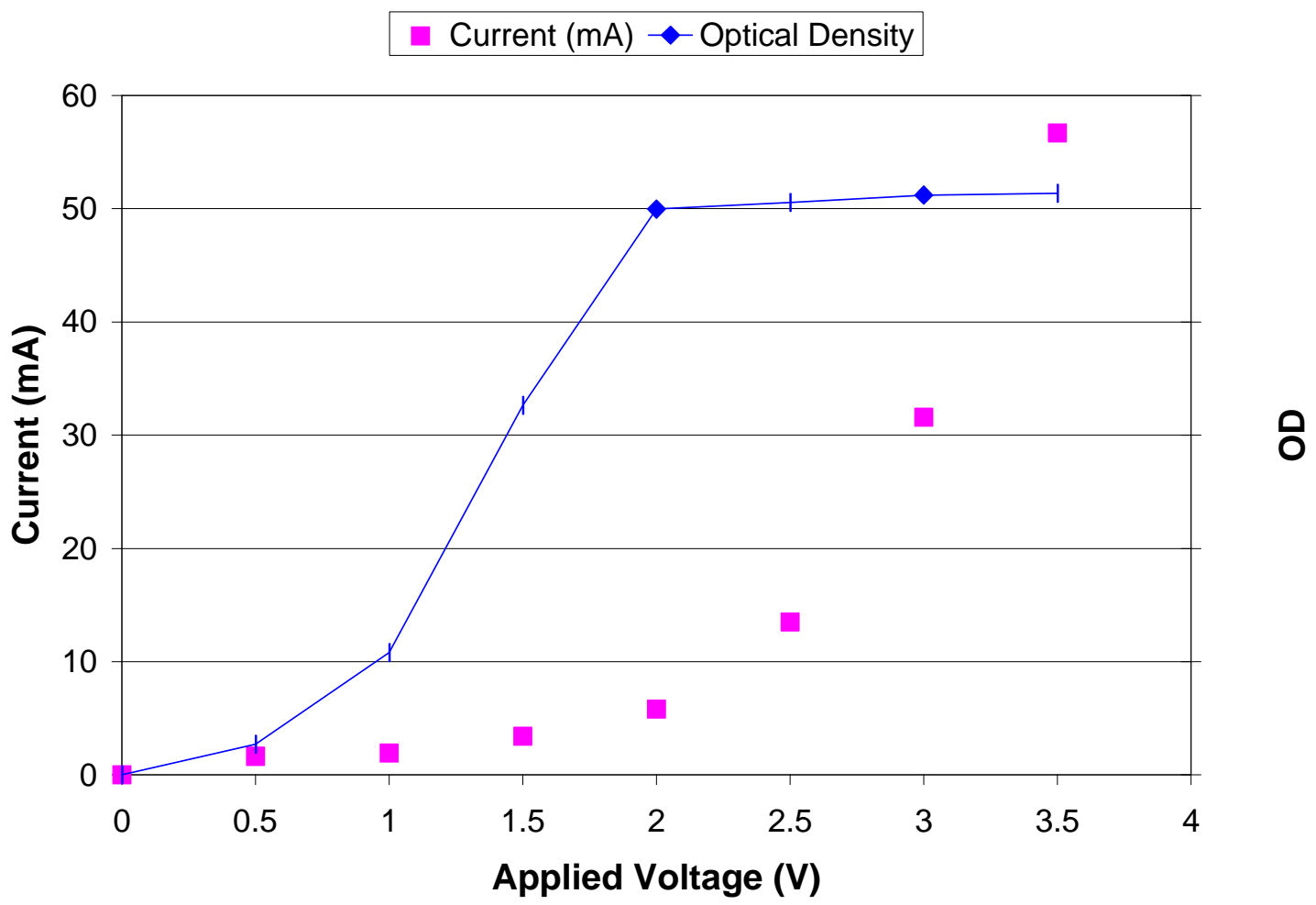

Figure 2. 14 - An example of electronic breakdown leading to saturation of the optical density of an EC device.

The experiments for optimizing the deposition process parameters have progressed to the stage where reasonable performance has been obtained. While fairly good performance has been achieved, as measured at initial test, some devices tend to 'slow down' during extended lifetime testing, causing them to be unsuitable for realistic applications. For example, devices typically color between $63-65 \%$ in the bleached state down to $2-5 \%$ in the colored state - an acceptable dynamic range - but these devices slow down to the point where it can take up to 4 hours to color fully after only a few hundred cycles. The reason will be the subject of further study.

\section{\$2.1.4 Develop Solid-state Model}

Understanding the electronic behavior of SageGlass ${ }^{\circledR}$ devices is important because it will allow determination of the parameters that dictate the electronic leakage currents through the stack, and also help in the design of a sputter deposited IC. In good devices, the leakage current appears to behave as if the device were a diode. An example of this is shown in Figure 2. 14. An understanding of the relevant parameters could conceivably allow the depth to which the device can be colored to be extended, thus increasing the dynamic range.

In this work, the origin of this diode-like behavior was investigated by constructing a range of samples with different structures in an effort to isolate the interface, or interfaces that lead to the observed electronic behavior. 


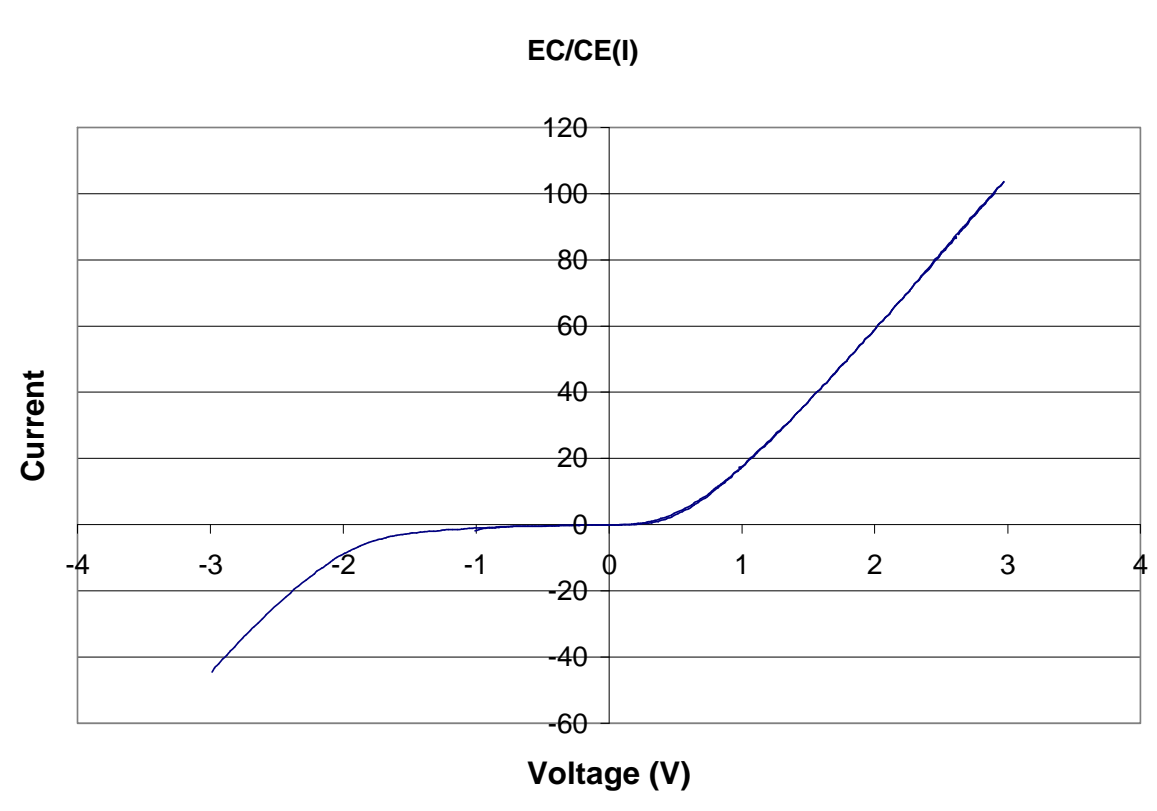

Figure 2. 15 - Example of electronic behavior of EC/CE junction, showing clear evidence of rectification in both forward and reverse direction.
Samples were

deposited with a

variety of

configurations, but it

was found that the EC

and the $\mathrm{CE}$ were

necessary for the diode

like characteristic to be observed. Samples of

EC/CE were deposited onto a conductive substrate and a transparent conductive top contact deposited to allow electrical measurements to be made. Rectifying behavior was seen in all the samples made, even where the CE thickness was

minimized. An example of this is shown in Figure 2. 15.

In all cases, the turn-on voltages were about $0.5 \mathrm{~V}$ for the forward direction, and $-2.0 \mathrm{~V}$ for the reverse direction. Variations in the thickness of the layers did not make any significant difference to these voltages as can be seen from Figure 2. 16. In addition, the slopes of the linear portions of the I-V characteristic subsequent to turn-on correlate extremely well to the resistance of the transparent conductors.

From this short study, it appears as though the origin of the diode-like behavior is the junction between the $\mathrm{CE}$ and the EC layer. Further proprietary modification of the $\mathrm{CE} / \mathrm{EC}$ junction yielded the very significant result shown in Figure A2. 3 in the Appendix. The forward turn-on voltage has been increased from $0.5 \mathrm{~V}$ to $\sim 1.7 \mathrm{~V}$ permitting deeper coloration and assuring the capability to switch larger devices.

The determining factor for the electronic response of SageGlass ${ }^{\circledR}$ devices is the junction between the EC and the CE. The measured turn-on voltages do not depend on the thickness of these layers. It was found that the IC stabilized this characteristic during subsequent processes. The exact mechanism for this is still unclear.

Generally, devices without IC do not switch well. Part of the reason may be related to the modification of the electronic barrier between the EC and the CE upon subsequent processing. Recent attempts to fabricate such devices generally end with a "leaky" system that does not switch well. An example is shown in Figure 2. 17. 
In contrast, three devices were made with a proprietary additive in the $\mathrm{CE}$ layer, again with no IC. The contrast in the electronic behavior is quite significant, and is shown in Figure A2.4 in the Appendix.

Significant progress has been made in understanding the electronic behavior of the SageGlass ${ }^{\circledR}$ EC system. The understanding is by no means complete, but a fundamental understanding of the critical process parameters has been gained. This has indicated that certain key process parameters must be maintained within critical limits, and there has been a concomitant increase in the consistency and repeatability of devices

Device with no IC

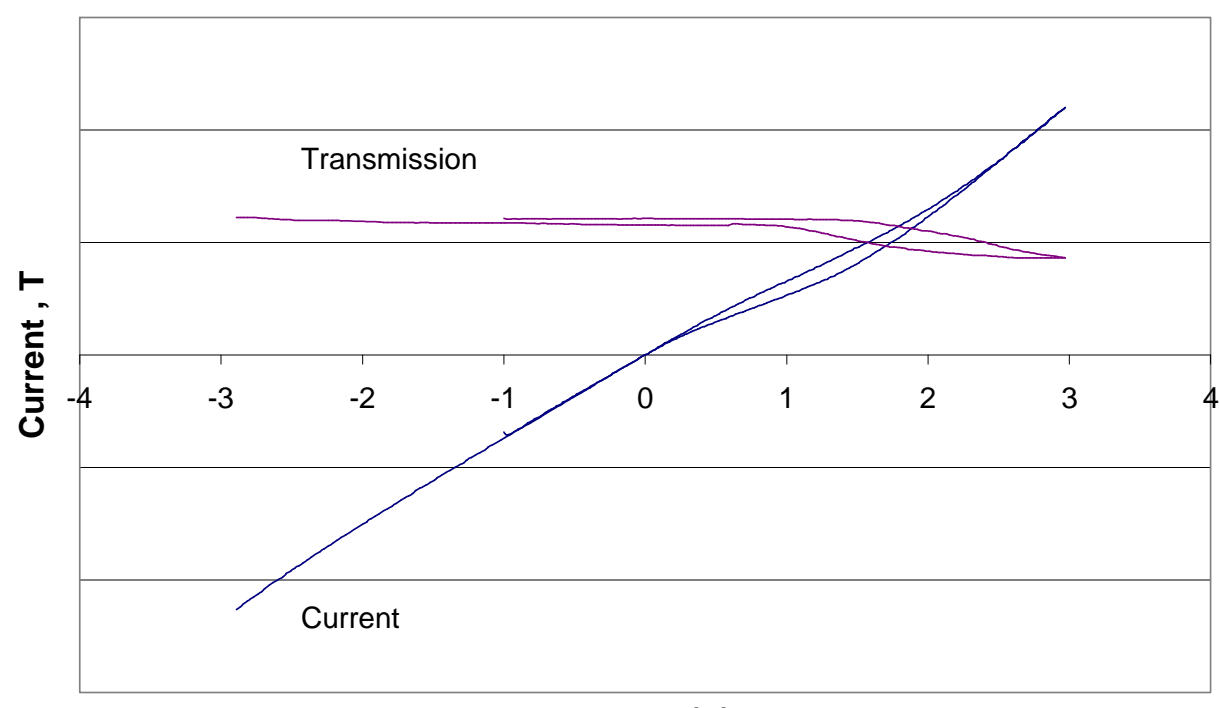

Voltage (V)

Figure 2. 17 - An example of a CV taken from an EC device made with no IC layer. Note the Ohmic behavior of the device, and poor coloration. produced by the pilot line as a result of taking this into account. This is particularly evident in the care taken with the level of lithiation, as this is known to have a critical impact on the device behavior. Work continues in an effort to further understand the influence of changes made to the various layers (i.e. thickness, processing temperatures, 
etc.) on the electronic properties of the device, particularly with a view to developing an alternative IC process.

\section{\$2.1.5 Increase switching speed}

It is well known that the switching speed of an EC device is limited by the sheet resistance of the TCO layers. This is because the voltage that can be applied to the device is limited and the amount of charge that must be transferred in order to fully color the device scales with the area. Consider a series of devices of fixed length ${ }^{\dagger \dagger}$, but with progressively larger and larger widths. As a device gets larger, or more precisely, as the separation between the busbars gets larger, the impedance of the EC stack itself - i.e. the part of the device where the current travels perpendicular to the surface of the glass - gets smaller. In contrast, the impedance of the TCO layers - where the current is flowing parallel to the surface of the glass gets larger. Overall, this change in area will lead to a larger potential drop in the TCO layers, either due to the higher current, or the higher impedance of the TCO layers themselves. Both situations will result in a lower potential applied directly to the stack, and so will result in slower switching.

In order to increase the switching speed of reasonably sized devices, i.e. suitable for architectural applications, it is necessary to increase the conductivity of the TCO layers. It is possible to increase the conductivity of the upper TCO by simply making it thicker, but this has two disadvantages: first, it introduces additional absorption, thereby reducing the bleached state transmission, and decreasing the dynamic range; second, it will cause the device to change color asymmetrically, where the color will appear from one of the bus bars and travel across to the other side of the device - a so-called 'curtain' effect). The curtain effect is undesirable because it is aesthetically unacceptable, and is likely to overstress the device at the point closest to the busbar.

The curtain effect can be avoided by ensuring that the sheet resistance of the two TCO layers is more or less matched, and it is conceivable that this can be achieved by depositing a further transparent conductor layer onto the substrate currently used, and thereby increasing the conductivity of both of the TCOs by the same amount. This has been tried, but all devices constructed this way have shown elevated leakage currents, and significantly reduced dynamic range. This is yet another indication of the interactions between the different layers, and is a problem which must be addressed if alternative substrates are to be used in the future.

However, if the size of the device is reduced to the size where the EC stack dominates the switching behavior, not the TCO resistance, then useful experiments can be carried out to understand the intrinsic limitations of the EC device. This work is particularly relevant to small devices that can be used as part of an optical system such as sighting mechanisms, mirrors or camera filters, where rapid response is required.

An experiment was carried out which attempted to determine the effect of lithium concentration on switching speed. It has been seen that samples with more lithium tend to switch more rapidly, and so small $(2.5 \mathrm{~cm} \times 2.5 \mathrm{~cm}(1 " \times 1 "))$ samples were made with different levels of lithium. It appeared that the rate of switching increased with increase in lithium level, but if the lithium level was increased too far the devices would become electronically leaky, and would therefore

\footnotetext{
${ }^{\dagger}$ The bus bars are assumed to run down the length of the device, and so an increase in width leads to an increase in the separation between the busbars.
} 
not color fully and the rate of switching would be reduced. There appears, therefore, to be an optimum level of lithium that maximizes switching speed. Further refinement of this is required to determine whether the onset of leakiness is gradual or sudden and also to determine if increase of the lithium level has any deleterious effects on any of the other important device properties, and this is ongoing.

\section{\$2.1.6 Summary of Improvements made to Materials Properties}

During this project, an enormous amount of progress has been made in the understanding of the fundamental behavior of the basic EC materials. Progress has been made in understanding the overall interrelationship between all the layers, and an electrical model has been developed. Some further progress has been made in identifying the key elements responsible for determining the overall switching speed of the device.

\section{§2.2 Task 2 - Rapid Conventional Heating}

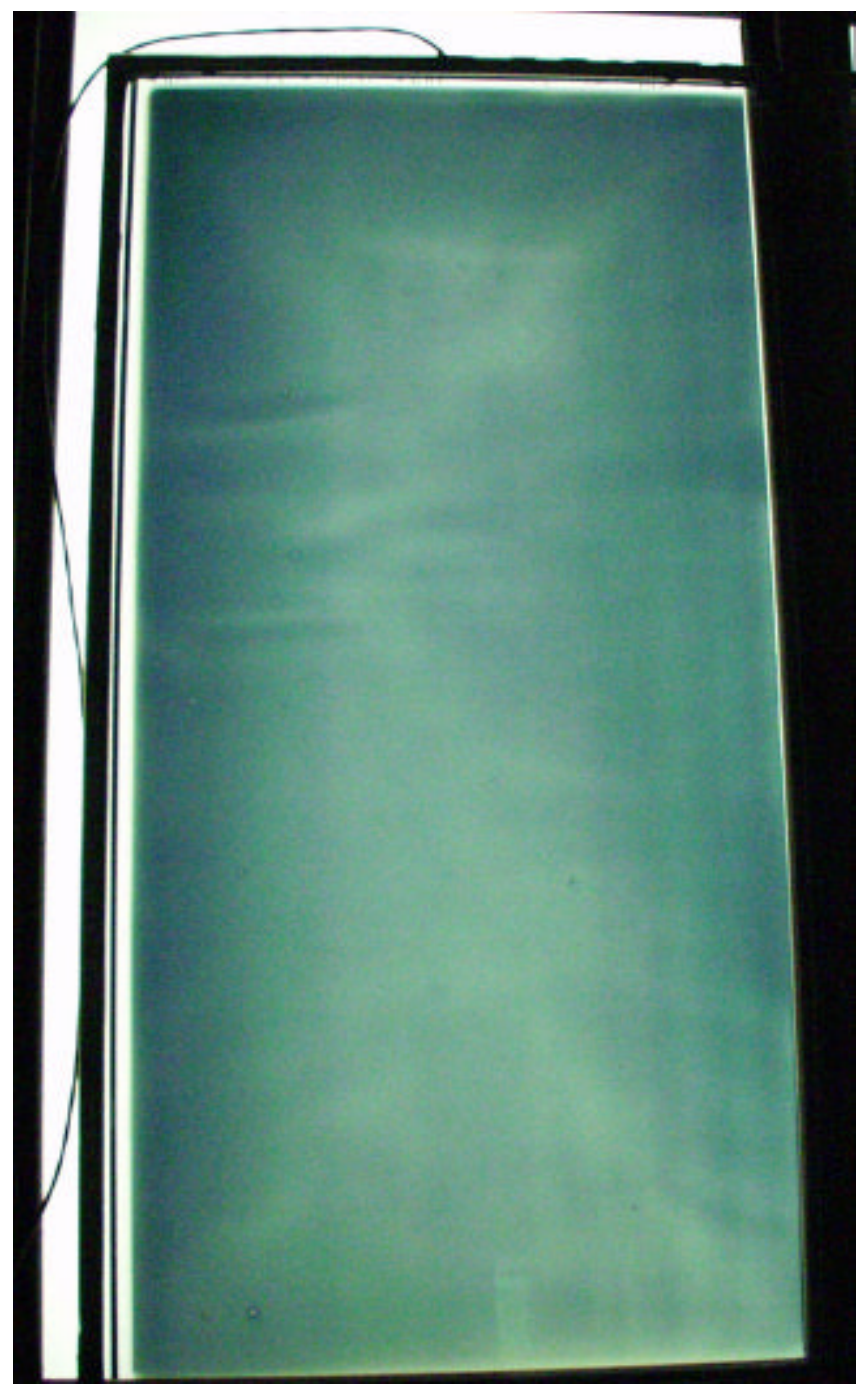

Figure 2. 18 - An example of the defect known as 'ribs'
In this section, the results from work aimed at improving the efficiency and yield of the thin-film deposition processes are presented, and in particular the heating steps. Much of the emphasis of the work in this area has been placed on thermal uniformity during the various heating processes. The variation seen across samples of various sizes during heat-up has been minimized by a concentrated effort.

The first oven used in the Pilot Line was a radiant oven using several banks of closely spaced quartz-halogen heating elements, which heated the substrate from above. The temperature of the glass was monitored using a pyrometer directed at a spot on the glass substrate on the opposite side to the heaters. This temperature measurement was used to control the power supplied to the heaters.

Unfortunately, several problems were found with this approach. The first thing that became apparent was the occurrence of a defect referred to as 'ribs'. A example of this is shown in Figure 2. 18, where an EC device is shown in the colored state during testing. (Note: This pattern is not evident in the clear state). The patterns of lighter bands can be clearly seen and 
cause the device to be rejected. Experiments were carried out to understand the origin of these bands, and although the exact mechanism was not determined, the cause was conclusively linked to the heating steps carried out in the radiant heater. It was proposed that the bands were somehow related to convection cells set-up in the heater, as a result of the largely static atmosphere inside the heater.

Several steps were attempted to try to reduce the convection, including inverting the substrate, so that the glass side was directly heated. These attempts almost always contributed additional complications. For example, inverting the samples led to poorly defined emissivity for the coated glass, and hence a temperature measurement which was unreliable. Naturally, this lead to inconsistent results for heating, and so eventually, the radiant heater was replaced with a forced air convection oven.

Additional problems had been found with the radiant heater, for example, when EC samples of different sizes were heated in the same process run they would display different electrochromic behavior. It was found that this was due to the way that low-e glass samples that are used as the substrates heat differently depending upon which side is exposed to the heat source, and how much radiant energy is being reflected from the surface opposite the heater. The low-e coating acts as a heat mirror, whereas the lamps used in this heater are designed to emit radiation that will be largely absorbed by the glass. It was found that with the samples placed coating side up (facing the lamps) in the oven, the coating reflected a high proportion of the incident radiation. However, a significant amount of heat was reflected off the bottom of the oven, leading to enhanced heating of the edges of the sample. This led to a significant edge to center variation of temperature for the samples and was more pronounced for the larger samples, leading to a reconsideration of the uniformity of the heating process.

The characterization of the uniformity was carried out by using a variety of sample sizes and orientations, gluing thermocouples in various positions to them, and running these samples through a thermal cycle. This gave a measurement of the uniformity for the various different sizes, and it was possible to minimize the variation by careful tuning of the temperature controller. As a result of this study, it was found that if the sample was oriented with the coating side down (away from the lamps), to maximize the amount of heat absorbed by the glass, and minimize the effect of the reflective low-e coating, much better uniformity was obtained. However, this caused the repeatability of the process to be compromised.

The repeatability of heating depends on the measurement of temperature was used to control the power supplied to the oven. A pyrometer was used in the oven to look at the amount of heat radiating from the hot glass surface. The amount of heat emitted depends upon the emissivity $(\varepsilon)$ of the surface. This is very well defined for the glass surface $(\varepsilon=0.86)$, but can vary dramatically for the coated side depending on what coatings are present. In addition, the emissivity for the same sample can vary between room temperature and typical processing temperatures, and so a single value (which is all that the pyrometer controller uses) cannot possibly describe the temperature accurately during the entire heating cycle.

It was therefore decided to abandon the radiant heater in favor of a forced air convection oven. This was installed and the process transferred to it in January 2003. An immediate effect was the removal of the ribs defect. Improvements in the repeatability and uniformity have been seen recently, and although there was no clearly identifiable improvement when the new oven was 
introduced, it is clear that it is one contribution amongst several to uniformity improvements, which has lead to an overall improvement.

The convection oven is a cross flow design with the substrate long dimension in the direction of airflow. The oven is a controlled atmosphere type with a sealed heating/cooling chamber. Heating is direct by electric heaters within the sealed chamber. The cooling is indirect with a large heat exchanger surface to remove heat from the sealed chamber.

Initial oven heating resulted in a mean substrate temperature within $5^{\circ} \mathrm{C}$ of the set point, which was deemed adequate. The range of temperatures across the substrate reached a maximum of $40^{\circ} \mathrm{C}$ at a mean temperature of $140^{\circ} \mathrm{C}$, and at the set point, the range was $20^{\circ} \mathrm{C}$. This was adequate at the time.

As part of the continual improvement program, process steps were regularly tested and their performance evaluated based on the measurable effects on electrochromic device performance. In March 2003 it was determined that other process steps had been improved to such a degree that the non-uniformity in the oven heating step had become a significant yield limiting factor.

A process improvement cycle was implemented for the oven-heating step. A series of experiments were carried out to determine the optimum combination of substrate orientation, substrate location within the sealed chamber, and the position of air diverters in the chamber. This work resulted in significant improvements, where the mean temperature repeatability of $5^{\circ} \mathrm{C}$ was maintained, while the uniformity across the substrate throughout the heating cycle was increased. The maximum range across the substrate was reduced to $15^{\circ} \mathrm{C}$ at a mean temperature of $120^{\circ} \mathrm{C}$. The range across the glass at set point was reduced from $20^{\circ} \mathrm{C}$ to $12^{\circ} \mathrm{C}$.

A reevaluation of the heating step after these changes showed that the effect of substrate temperature non-uniformity was now below the measurable level based on present range of electrochromic device performance.

As an illustration of this, an average measurement of uniformity for $25 \mathrm{~cm} \times 30 \mathrm{~cm}(10$ " $\times 12$ ") taken during September of 2001 was $\Delta \mathrm{E}=(7.3 \pm 4.0)$, whereas a similar measurement for May of 2004 was $\Delta \mathrm{E}=(2.1 \pm 0.7)$. This demonstrates both an improvement in the uniformity of individual sample, and also an improvement of the run-to-run repeatability. Once again, it is noted that the improvement cannot all be attributed to the improvement in the uniformity of the heating process, but it obviously played a role.

An additional issue with the original IR heater was throughput. Only one load of glass of approximate area 40 " $\times 40$ " could be processed at one time, causing the oven to become a bottleneck. The introduction of the forced air convection oven increased the amount of glass capable of being processed at any one time by a factor of approximately five. This batch type operation will be retained for the manufacturing plant, as it has been determined that it is possible to 'stockpile' enough samples to fill the oven without causing any performance degradation.

\section{\$2.3 Task 3 - Operational testing}

The aim of this task was to address some of the process bottlenecks that were anticipated in the area of device testing, as the process was scaled-up towards production volumes. This included development and construction of high throughput large area device test facilities. In addition, if 


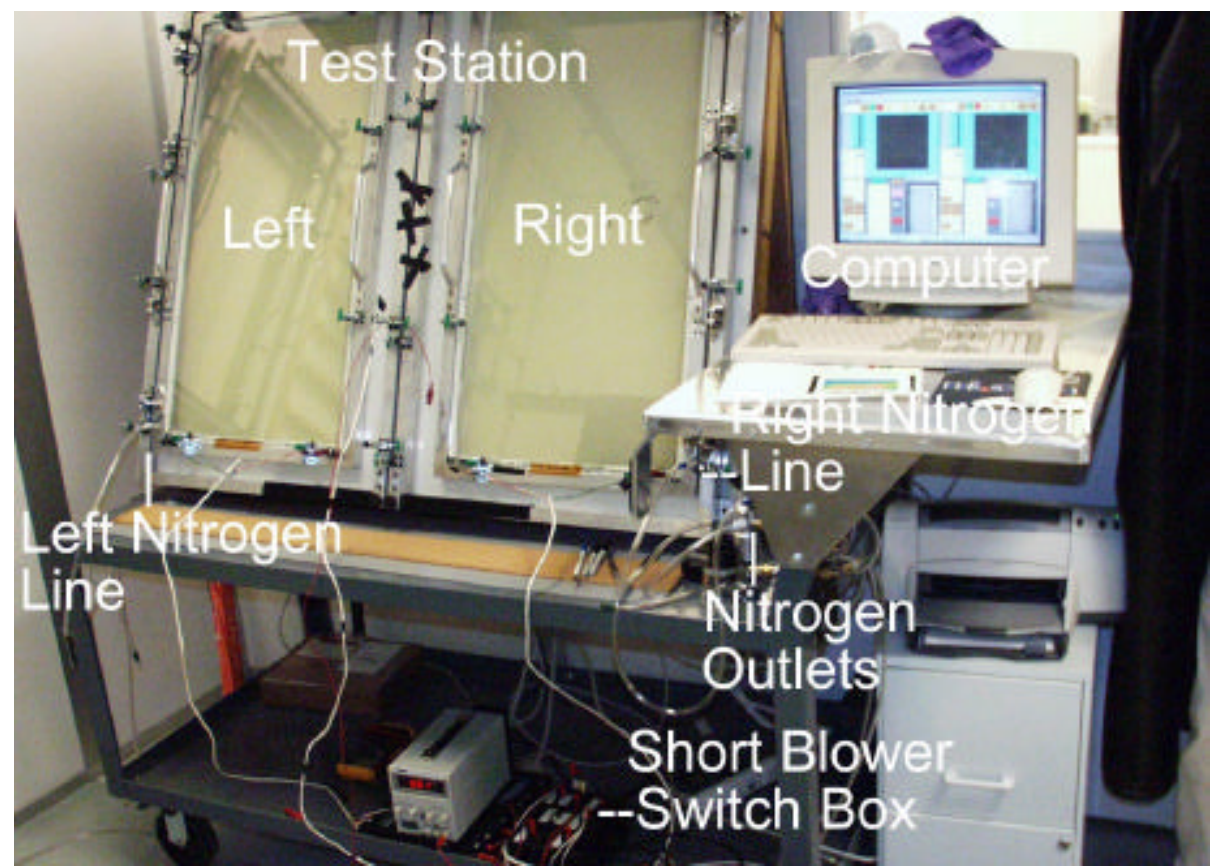

Figure 2. 19 - The updated monolithic device tester, shown configured to measure two $46 \times 89 \mathrm{~cm}\left(18 \times 35^{\prime \prime}\right)$ samples. The backlight is turned off for the photograph. an electro-optical device model could be developed from the test measurements, then it could conceivably be parameterized, and used to generate data used for controller calibration.

The final device test in SageGlass ${ }^{\circledR}$ manufacture will perform two distinct functions: (i) Quality Control and Assurance, and (ii) Control System Calibration. At the start of the project,

testing was a bottleneck, and little was known about the opto-electronic device characteristics. Both of these issues needed to be resolved, and the results from these activities are described in the following sections.

\section{\$2.3.1 Design and Develop Prototype Test Station}

At the start of the project, the test and characterization facilities at SAGE were rudimentary, and provided measurements of the transmission and electronic characteristics of devices with some difficulty. The transmission monitor consisted of a modified camera that contained a $4 \times 5$ array of photodiodes, giving a crude measurement of uniformity. Changes of device size were not easy to accommodate, and the uniformity of the backlight lead to problems with characterization.

An updated tester was commissioned at the start of the project, with the additional requirements compared to the existing facility of being able to measure color coordinates, coloration uniformity in both the colored and the bleached states and to measure the transmission of arbitrarily defined areas of the device. All of this has become feasible with the advent of cheaper higher resolution digital cameras. In addition, it has been constructed so that it can measure a number of different devices simultaneously.

The devices to be tested are placed in front of a uniform white backlight, and connections are made to a computer controlled DC power supply. Different device sizes are easily accommodated by using a modular frame system, where each device size has a frame used to hold the device securely, and be fixed to the tester simply. Each frame is also hinged to allow access to the front (i.e. film side) of the glass to allow rework of defects to take place.

The tester is shown in Figure 2. 19, where two $46 \times 89 \mathrm{~cm}(18 \times 35$ ") devices are shown ready to be tested. The computer to the right is used to control the power supplied to the device as well as 
to measure electrical and optical properties. A nitrogen purge is used to maintain an inert atmosphere for device tests, to minimize the effect of moisture on the devices.

The computer control has been developed so that it is simple to use, but versatile. It is also networked, and so measured data is saved in a centrally accessible database, and is therefore available to allow correlations between process parameters and device performance. The user interface is shown in Figure 2. 20. A wide range of functionality is immediately apparent and this level of sophistication can allow the operator to perform tests on several different devices at the same time on the same tester. Currently, the software, and some of the electrical hardware limits this to two devices per tester, although there is no fundamental limit to this. The limiting factor is the backlight size, which was designed to accommodate two $48 \times 94 \mathrm{~cm}\left(19 \times 37^{\prime \prime}\right)$ devices at once.

The monolithic test is also used to assess device defect levels, and the device can either be scrapped, passed on to the next stage of the process - IGU fabrication - or the defects can be 'reworked'. Rework is a process that allows certain defects to be removed cleanly from the device, thereby increasing the overall yield. Simple techniques for detecting shorts have been developed, but current methods of removal require significant skill on the part of the operator. Automatic methods involving vision systems are currently being considered for the future.

Under this contract, several additional large device testers were built with expanded capabilities for device characterization. An important objective was to develop the capability to test full size EC devices over a range of temperatures. Another important consideration was to allow the operator to test more devices, either by automating the tests somewhat, or by shortening the whole test procedure. 


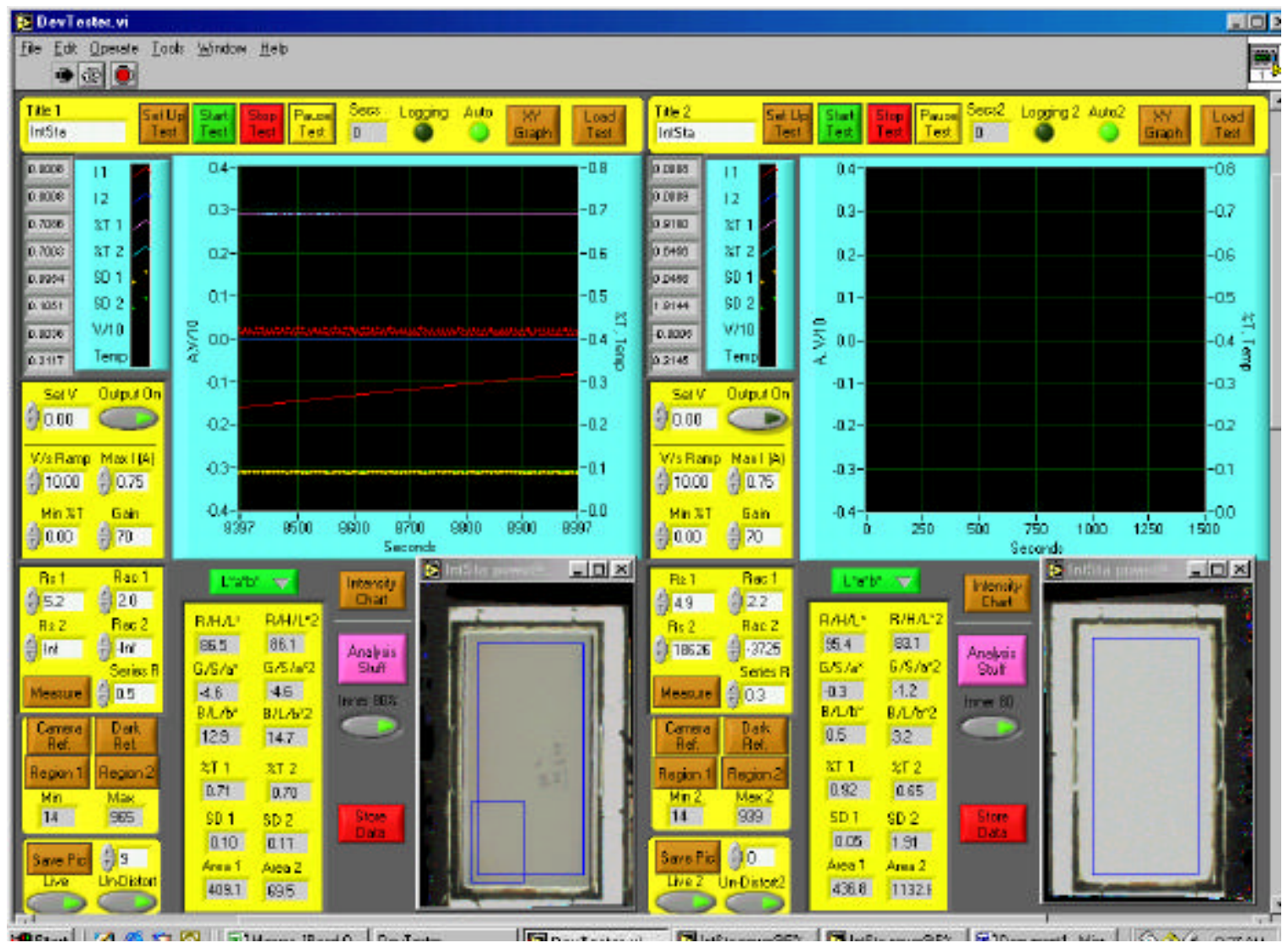

Figure 2. 20 - The user interface of the device tester, showing the wide range of measurements available, including current, voltage, transmission, color and even temperature.

The following sections give a summary of the work done in this area.

\section{§2.3.1.1 Temperature Control}

The most significant hardware improvement was the addition of temperature control for the device under test. To accomplish this, a five-inch-deep thermally insulated enclosure was placed in front of the backlight, with air circulated through heating and/or cooling elements.

For cooling, a liquid chiller cools a mixture of water and propylene glycol, which is circulated through an air/liquid heat exchanger. For heating, an electric heating element directly heats the air. A blower, used for both heating and cooling, circulates air through the insulated enclosure, heat exchanger, and heater.

The electrochromic insulated glass units (IGUs) are placed in the front of the enclosure, with the active side toward the inside of the enclosure. In this way, the test system represents a somewhat realistic environment, with the active side "outdoors" (significant temperature range) and the inactive side "indoors" (consistent temperature.)

With this new system, it is possible to thoroughly test various IGU sizes over a temperature range from below $15^{\circ} \mathrm{C}$ to at least $65^{\circ} \mathrm{C}$. While this temperature range is more limited than actual field conditions, it is sufficient to allow development of a device model that incorporates temperature effects. Without this piece of equipment, this would have been nearly impossible, as previous test systems with temperature control were inside environmental-control chambers, 
allowing only a couple of spotmeasurements of light transmission via fiber optics. Spot measurements, while helpful in some contexts, do not provide enough information to accurately develop a device model.

Another helpful application of this test system is its ability to show device kinetic

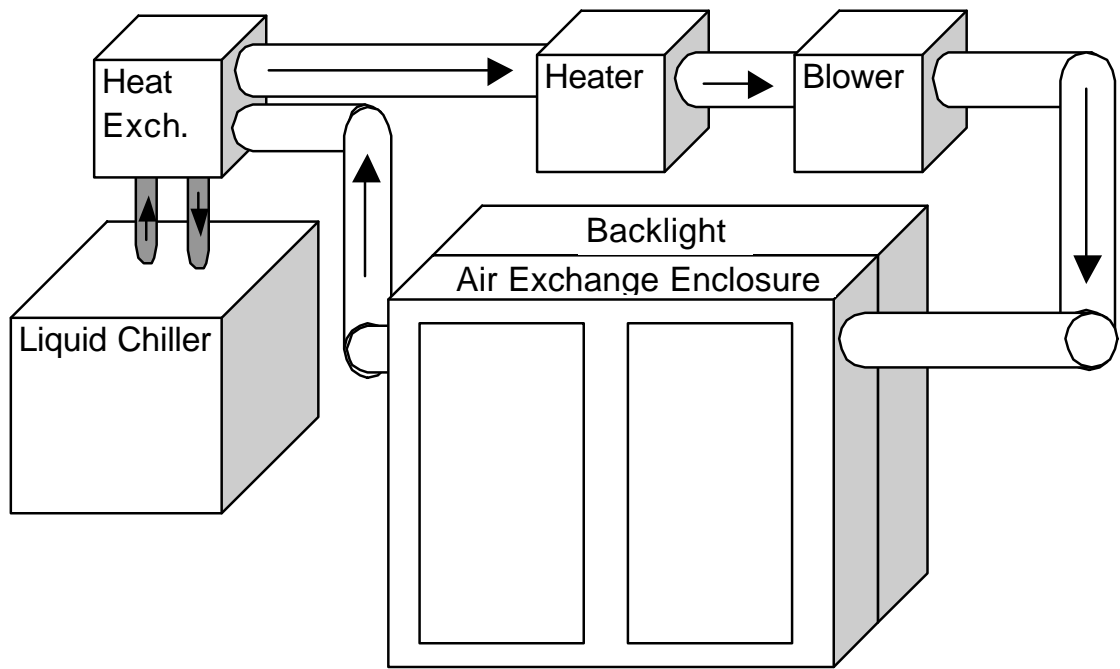

Figure 2. 21 - Schematic figure of heating and cooling set-up for the device tester performance over a range of temperatures. Some non-uniformity in electrochromic devices is temperature-dependent, making it hard to fully understand the effect on user perception without this sort of test system.

\section{\$2.3.1.2 Test Sequencing}

The "looped" test simply runs a sequence of operations over and over until interrupted by the operator. This could be used, for example, to alternately color and bleach a device, holding it for a specified period of time in each state. This has been used, for example, to investigate device properties that seem to change during the first few cycles.

Finally, any test set up by the operator may be saved as a script file (using the "Load Sequence" and "Save Sequence" buttons shown in the lower left corner of the dialog box.) In this way, an operator may create a test sequence that is used repeatedly with a minimum of set-up work.

The sequencing of tests has also been significantly improved, allowing more rapid, efficient testing of electrochromic devices. The Figure to the right shows the dialog box used to set up individual and sequential tests. 
The "Mode Select" element in the upper-left corner of the image is used to select an automatic, manual, sequenced, or looped test. Automatic and manual tests have always been available. Manual tests simply log data while allowing the operator full control of applied voltage and transmission limits.

Automatic tests apply a sequence of voltages, voltage ramps, or transmission limits.

The new "sequential" mode allows the operator to set up a series of discrete tests which will be performed in sequence, saving the data to separate files, as shown in the "File Path" box, and as many as five sequential tests may be run.

\section{\$2.3.2 Rapid Test Methods}

As production levels are increased to higher volumes, it is important that the testing capability both at monolithic level as well as after durability screening - maintains pace, and therefore does not
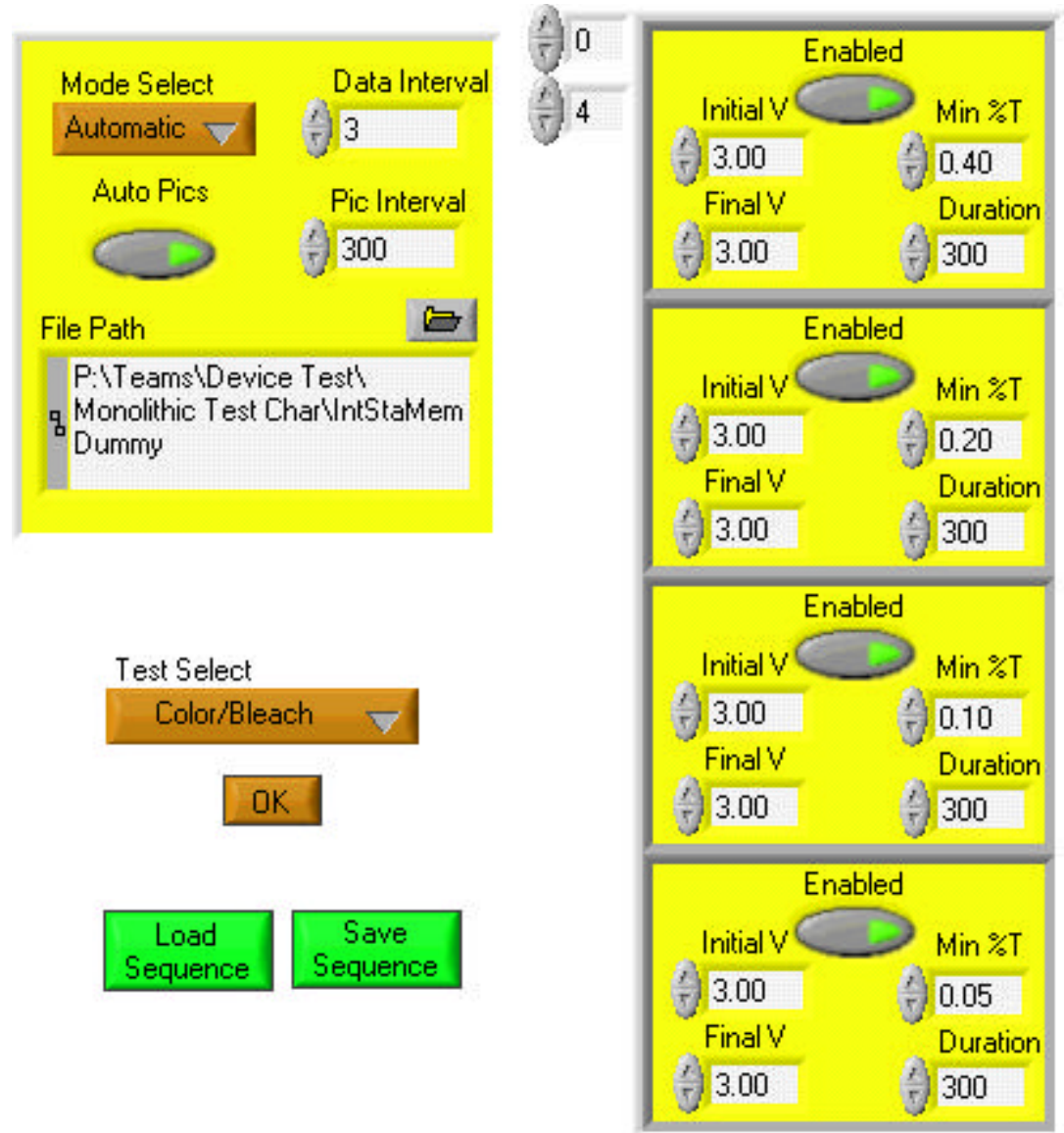

Figure 2. 22 - Test Dialog Box become a bottleneck.

The first test that the device is subjected to after fabrication is a monolithic performance characterization.

Some automatic data entry functions have therefore been added to the tester software to facilitate faster computer data entry. These functions have decreased the amount of time needed for test set-up, as well as made it easier for the operator to retrieve process information vital to understanding the test results. Automatic data entry has also decreased the time needed to collect and enter data during the test, although having little effect in the total test time, it does free-up time for the operator to run additional testers, which will become significant when these are added in the future.

\section{\$2.3.3 Electro-optical Device model}

Development of a model utilizing a small set of measured fundamental device parameters describing all the key performance properties and able to distinguish device likely to be durable from those likely to degrade over time would be an enormous benefit. Ideally, this model would describe electrical and optical response to applied electrical power, over the useful range of 
transmission levels and temperatures. The following section describes the work carried out in this area.

\section{\$2.3.3.1 Introduction}

The objective of this work is to determine a set of fundamental device parameters that can be used to describe all the key performance parameters of an EC device. These parameters would be most useful if they could be determined from a simple characterization test. Another potential use would also be to use these parameters to distinguish devices that are likely to degrade over time. Successfully achieving these objectives would dramatically reduce the amount of testing and characterization required, help in developing control algorithms and reduce potential degradation of samples in the field.

To accomplish these objectives, it was necessary to develop an understanding of the electrooptical properties, yielding a parameterized model. This model is required to describe the behavior of EC devices as a function of temperature as well as the room temperature response. The work focused on developing and refining an equivalent circuit model based on standard electrical circuit elements. Also, an understanding of the solid-state physics and electrochemistry of the device was developed - this is discussed in Section \$2.1.4- which helped to illuminate the dependence of the circuit element model on physical properties of the EC devices.

Initially, the behavior of EC devices at constant temperature was considered. Two important relationships were derived from measurements of SageGlass ${ }^{\circledR}$ windows at equilibrium (that is, under conditions of steady-state voltage, current, and transmission). These are the relationship of voltage to electronic current (at equilibrium there is no ionic current) and the relationship of voltage to transmission state.

Transmission state, in this document, is given in units of OD. Voltage will be given as "internal voltage," which is an approximate measurement of the voltage applied to the electrochromic stack; that is, the applied voltage less the voltage drop across the transparent conductors and bus bars. The purpose of using "internal voltage" is to minimize the size-dependence of the measurements.

\section{\$2.3.3.2 Theoretical Understanding}

The following is a discussion of the current understanding of the theoretical principles concerning the parameters discussed in previous sections. Figure 2. 23 shows a schematic (model equivalent circuit) for the EC device which has been deduced from studies of the electronic behavior of EC devices. This represents a single element of the device, and in order to explain the behavior of a large area device, this could form the basis for one spatial element of a finite element model.

Essentially, the model can be split into two parts: an electronic pathway and an ionic pathway. The measured current will be the sum of these two components, and the main challenge in understanding (and hence the ability to control the device) is in disentangling the two components.

The applied voltage $\left(\mathrm{V}_{\text {app }}\right)$ causes a current $\left(\mathrm{I}_{\mathrm{app}}\right)$ to flow into the device through the series resistance (primarily made up of the transparent conductor sheet resistances and the bus-bars). This current then is split into the ionic and electronic components. It has been found that the 
electronic current ( $\left.\mathrm{I}_{\text {leak }}\right)$ follows a path that appears electronically to be equivalent to a diode in parallel with a resistor. ${ }^{*}$ The ionic path appears to be equivalent to a resistance in series with a charge storage element. The following sections describe some characteristics of the equivalent circuit in more detail.

\section{§2.3.3.3 Breakdown Voltage}

The "breakdown" behavior (associated with the leakage diode in the electronic pathway) appears to be a property of the junction between the $\mathrm{CE}$ and the EC. The breakdown behavior is expected to be dependent on the material properties of the EC, IC and CE layers, and on the interfaces between them. It is noted, however, that varying the thickness of the layers does not appear to significantly change the breakdown voltage, and that the behavior is asymmetric (different for coloring and bleaching potentials).

\section{\$2.3.3.4 Voltage Efficiency}

Voltage efficiency (change in optical density per voltage increment) seems to be, in large part, a measure of the "battery capacity" of the electrochromic stack. Consider, for example, the difference between a $C$ and $D$ cell. More charge is required to charge a $D$ cell to a given voltage because of its large capacity. Replace "charge" with "optical density" in that sentence and one can see that a D cell has a higher voltage efficiency (more charge per volt). This has been seen in some experiments where devices with 2 and 6 passes of $C E$ have been made, rather than the more typical 4. Devices with 2 passes of CE had voltage efficiencies less than 0.5 . Those with 6 passes were generally between 1.2 and 1.5. It is also probable that lithiation affects voltage efficiency. Again, this is not surprising, since the "battery capacity" is a function of EC, CE, and lithium. It appears that, at the moment, $\mathrm{CE}$ is more of a limiting factor for coloration than EC, so variations in $\mathrm{CE}$ have more effect on voltage efficiency. Leakage variations also appear to have some effect on this measurement, though this is not well established or quantified.

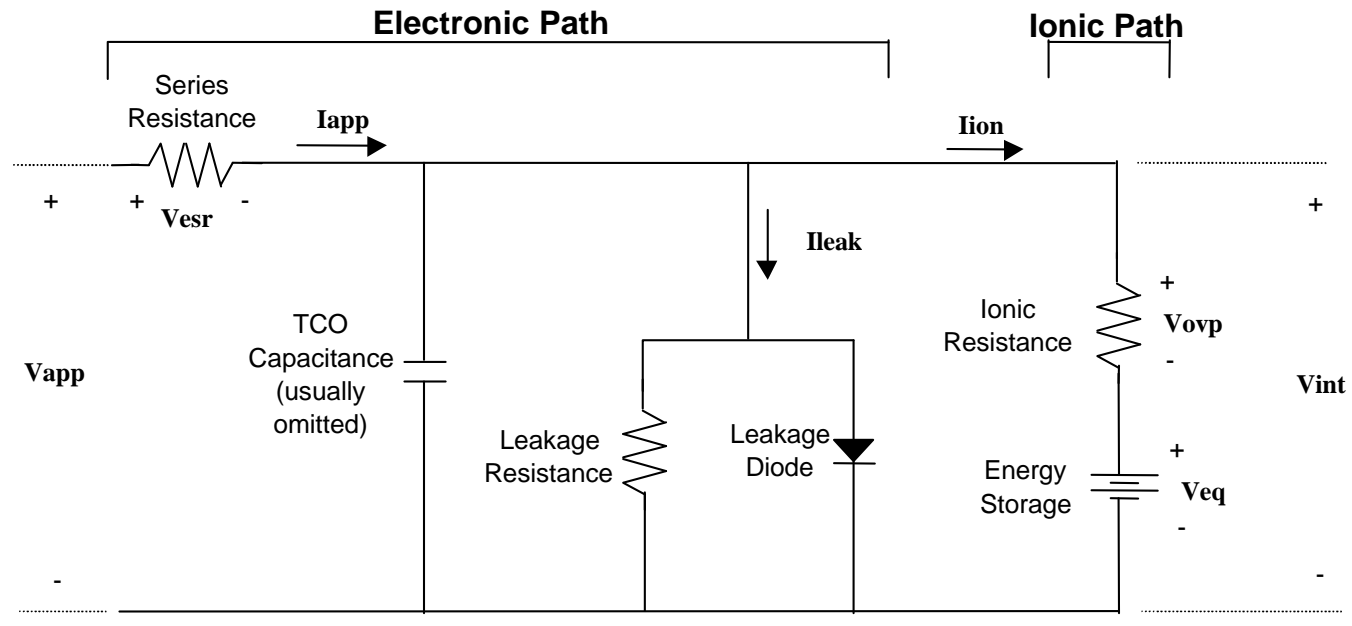

Figure 2. 23 - Device Schematic Model (Equivalent Circuit)

\footnotetext{
${ }^{*}$ It is possible to increase the sophistication of the model by including two such circuit elements, with two diodes oriented in opposite directions with different breakdown voltages, reflecting the different breakdown voltages seen in the forward and reverse directions.
} 


\section{\$2.3.3.5 Threshold Voltage}

The threshold voltage (voltage required to begin coloring a device) is a function of electrochemical potential of the EC and CE, which can be affected by the amount of incorporated lithium, and probably deposition conditions and heating of the various layers (during and after deposition). There is very little hard data at the moment to extract much meaning from this parameter, except that lower thresholds appear to be better from a performance standpoint.

\section{\$2.3.3.6 Leakage Resistance}

Leakage resistance appears to be dominated by defects-devices without defects have a very high leakage resistance, which is to say very low electronic current at voltages below breakdown. It has been speculated that all leakage is a matter of defects-perhaps micro-defects that are not visible. It is clear that the potential for very low leakage current is achievable with the present stack.

\section{§2.3.3.7 Summary of Electro-Optic Device Model}

Significant progress has been made in the understanding of the electro-optical behavior of EC devices. Indeed, several terms have been invented (such as voltage efficiency) to describe the behavior that has been observed. The lumped parameter model is fairly well established, although areas where the level of sophistication can be increased have been identified. At least one of the parameters (the series resistance) is already used to monitor the evolution of the device performance as it undergoes durability testing. This measurement is simple, and can be used to determine whether the physical integrity of the device has been compromised without the need to perform a full color-bleach characterization.

Future work will focus on the detailed understanding of the model, and the relationship of variations in the EC film deposition process to elements within this parameterized model. In addition, the temperature dependence of EC devices will be studied in more detail, and the consequences for design of practical controllers and control algorithms determined.

The original aim of the work was to increase the testing throughput to levels suitable for manufacturing, and also develop a device model that would allow rapid test algorithms to be developed. The highly complex nature of the behavior of the EC devices has prevented this from being completely accomplished, but several steps have been taken which have laid the groundwork for future progress.

\section{§2.3.4 Manufacturing Process Improvements}

This section covers some of the improvements made during this project on the manufacturing methods used to produce SageGlass ${ }^{\circledR}$. In particular, focus is placed on one of the important quality programs introduced during the work, that of Design of Experiments (DOE). This is a powerful technique for process optimization, and it finds applications in optimizing the thin-film deposition processes, and here it is considered for that purpose and also as a means to optimize device fabrication processes, i.e. processes concerned with turning the monolithic EC-substrate into an EC-window suitable for installation into a building or vehicle.

In addition, the use of lasers for various different applications was investigated. The results of trials aimed at several different tasks are discussed. 


\section{§2.3.4.1 Utilize DOE to optimize fabrication processes}

Design of Experiments (DOE) is a planned approach for determining cause and effect relationships that can be applied to any process with measurable inputs and outputs. DOE provides a statistical means for analyzing how numerous variables interact. Designed experiments require some up-front planning to be successful, as it is possible - even desirable to study several different variables in one set of experimental runs. The generic name for this approach is "Factorial Design," and this will be briefly explained below. Coupled with the factorial design is the statistical analysis of variance (ANOVA), which allows dependencies and interactions between variables to be cleanly determined from the measured data.

\begin{tabular}{|c|c|c|}
\hline & Factor 1 & Factor 2 \\
\hline 1 & HIGH & HIGH \\
\hline 2 & HIGH & LOW \\
\hline 3 & LOW & HIGH \\
\hline 4 & LOW & LOW \\
\hline
\end{tabular}

Table 2. 2 - Two-factor two-level design
The factorial design approach is in complete contrast to the more usual one factor at a time (OFAT) experimentation methodology, where only one factor is varied and all the others are held constant. In the factorial design approach, interactions between variables are revealed where they would be missed in the OFAT approach. In addition, the number of experimental runs required to investigate a certain volume of experimental space is reduced compared with OFAT, thus increasing the experimental power. The experimental power increases with an increase in the number of variable factors.

In general, experiments have been limited to two-level factorial designs. Using this method, two levels (HIGH and LOW) are chosen for each variable factor. In a two-factor two-level design (Table 2. 2), there are therefore four unique combinations. In a three-factor two-level factorial (Table 2. 3), there would be eight unique combinations of variables.

An experimental design would therefore run at least one sample with each combination, and the results from any measurements analyzed using the ANOVA approach. Naturally, the entire experiment can be replicated to give better statistical selectivity. Essentially, there would be four pairs of experimental points (1 and 5, 2 and 6, 3 and 7, and 4 and 8) which can be compared with each other where the only difference between the pairs is that (for instance) factor 1 is HIGH or LOW. If factor 1 is significant, then each of the pairs should show the same trend, so there are four experiments probing the significance of factor 1 .

However, the factor could be involved in an interaction meaning that one factor depends on the value of another. The ANOVA approach allows such interactions to be extracted from the measured data in a simple and clear way. Obviously, the effectiveness depends on the initial experimental design.

An additional benefit of the DOE approach comes as the complexity of the system

\begin{tabular}{|c|c|c|c|}
\hline & Factor 1 & Factor 2 & Factor 3 \\
\hline 1 & HIGH & HIGH & HIGH \\
\hline 2 & HIGH & HIGH & LOW \\
\hline 3 & HIGH & LOW & HIGH \\
\hline 4 & HIGH & LOW & LOW \\
\hline 5 & LOW & HIGH & HIGH \\
\hline 6 & LOW & HIGH & LOW \\
\hline 7 & LOW & LOW & HIGH \\
\hline 8 & LOW & LOW & LOW \\
\hline
\end{tabular}

Table 2. 3 - Three-factor two-level design 
increases. As the number of variable factors increases, the number of unique combinations or experimental points goes up accordingly. However, fractional factorial experiments can actually be run without losing any statistical resolution, where only a subset of the experimental combinations is required. For example, the Table 2. 4 shows the savings - meaning the number of redundant combinations - that can in principle be made by using fractional designs.

\begin{tabular}{|c|c|c|c|}
\hline $\begin{array}{c}\text { Number of } \\
\text { Factors }\end{array}$ & $\begin{array}{c}\text { Full } \\
\text { Factorial }\end{array}$ & $\begin{array}{c}\text { Fraction } \\
\text { required }\end{array}$ & Savings \\
\hline 4 & 16 & 12 & $25 \%$ \\
\hline 5 & 32 & 16 & $50 \%$ \\
\hline 6 & 64 & 32 & $50 \%$ \\
\hline 7 & 128 & 32 & $75 \%$ \\
\hline
\end{tabular}

The interested reader is referred to "DOE

Simplified," by Anderson and Whitcombe (2000) Pub: Productivity Inc. ${ }^{19}$, or "Design and Analysis of Experiments," Montgomery, (1997), Pub: Wiley, ${ }^{20}$ for further details on all aspects of DOE.

Full factorial designs, usually with two or three variable factors, have generally been used. The DOE methodology has

Table 2. 4 - Fractional factorial designs been adopted completely, and is now used for process optimization experiments. The results of several of these experiments are discussed in the section on the ion conductor studies (Section $\$ 2.1 .3$ ).

\section{\$2.3.4.2 Laser patterning}

Several opportunities exist for laser patterning within the EC device fabrication process. There are many areas where lasers can be of use in the EC device fabrication process, but this project is restricted to two specific areas: isolation line patterning, and isolating different areas of the substrate to produce multiple switching areas on the same glass substrate.

At the start of the project, the line isolation -required to prevent the two conductive bus bars printed on the substrate from shorting to one another - was produced by sandblasting. This was a poorly controlled process, resulting in a relatively wide, irregular and rough abraded area. This led to problems with excessive leakage through the film stack at the line isolation because of the difficulty in ensuring adequate film coverage over the rough edges of the line isolation.

Patterning of transparent conductors using lasers is well known within the photovoltaic industry, and so it was thought to be appropriate here. In this application, a laser would simply be required to remove the FTO from the substrate over a narrow region, leaving two isolated regions.

In the second application, the laser will be used to remove films so that multiple smaller devices can be fabricated on a single substrate. In this way, device areas can be isolated, and switched individually, leading to the possibility of fabricating display elements or smaller EC elements, such as filters for high precision optical instruments.

\section{Isolation line fabrication}

A commercially available Nd:YAG laser (1.06 micron wavelength) was used for isolation line fabrication. The system is generally used for routine laser marking applications. Properly focused, the laser has a minimum line-width of 25 microns with a pulse repetition frequency of 0.1 to $50 \mathrm{kHz}$. The effects of focus height, power and raster area on damage zone size were examined. 
Four different power settings (90, 80, 70 and $60 \%$ of maximum power) were used, and lines were fabricated with the TCO layer up as well as through the glass. The lines fabricated with the TCO layer up (100 microns wide ${ }^{\S \S}$ ) were approximately $2 \times$ narrower when compared to similar lines fabricated through the glass layer; this could be explained by beam expansion due to small errors in focusing. All lines made using greater than $60 \%$ maximum power resulted in good electrical isolation (resistance across the lines greater than $40 \mathrm{M} \Omega$ ).

Subsequently, devices were fabricated on these laser-isolated substrates, and the leakage currents were found to be somewhat lower than for devices with a sandblasted line isolation fabricated in the same run. Clearly, laser line isolation is practical, and is something that can be incorporated into the production process as soon as a laser is obtained.

\section{Further Isolation Trials and Area Isolation}

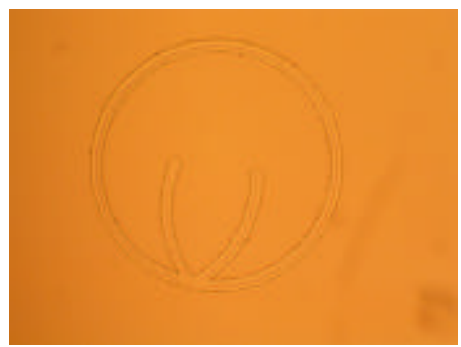

Figure 2. 24 - 100x photomicrograph of a $>2 \mathrm{M} \Omega$ isolation circle scribed in the first transparent conducting oxide (TCO).
Additional laser scribing trials were conducted with an ESI 5200 laser scribe table equipped with a q-switched, frequency tripled, Nd:YAG laser. The laser was operated at close to its lowest power output, at 0.4 and 0.8 Watts, at a pulse rate of $20 \mathrm{kHz}$, with typical pulse durations of $40 \mathrm{~ns}$. A test pattern was scribed through the coatings, consisting of an array of $1 \mathrm{~mm}$ diameter circles, a $3 \mathrm{~mm}$ circle, and a $6 \mathrm{~mm}$ circle. The electrical isolation of complete circles scribed through the TCO layer, as shown in Figure 2. 24 was found to exceed $2 \mathrm{M} \Omega$. When scribed through the entire EC stack, the laser scribing yielded complete isolation of the EC performance, both coloration and bleaching, with no bleeding of contrasting light or dark areas due to Ohmic drop, as shown in Figure 2. 25.

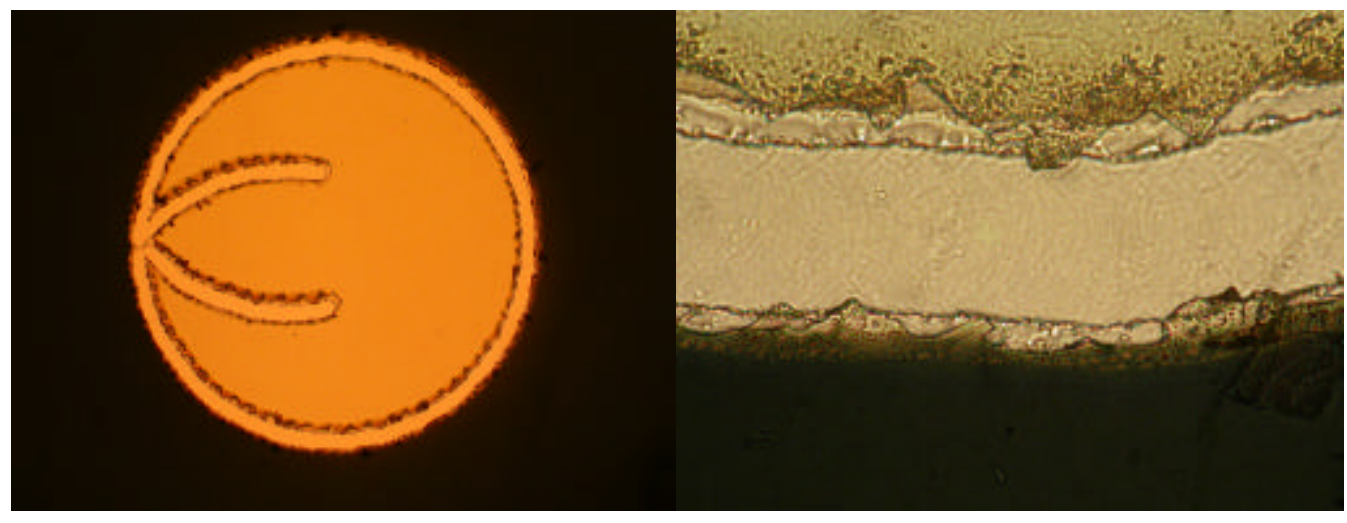

Figure 2. 25 - Photomicrograph of laser patterning through the entire EC stack. The surrounding films could be colored and bleached independently of the isolated circle, which remained bleached. The left is at a magnification of $100 x$, while the right is taken at $1000 x$.

\section{Multiple Switched Areas}

Laser pattering was used to produce an array of small devices spaced a nominal distance of $5 \mathrm{~cm}$ (2”) apart. This was very successful, in that the isolation was both clean and very narrow, leading

\footnotetext{
$\S \S$ This should be compared to linewidths of around 500 microns for typical sandblasted lines.
} 
to excellent isolation and very good visual qualities. However, the one significant problem in laser processing the $5 \mathrm{~cm}$ (2") square pattern was the exact spacing of the squares. Dimensions varied by typically up to $0.08 \mathrm{~cm}(0.03$ "). A significant effort by the firm responsible for producing the laser patterning has tightened this significantly, and now the error in the dimensionality is estimated to be approximately $0.0025 \mathrm{~cm}(0.001$ ”).

\section{Summary of Laser Processing}

Clearly, laser processing is practical for EC device fabrication, and will be incorporated into the process as it is scaled-up. The choice of laser system will most probably be determined by other requirements such as defect rework, as it appears that substrate patterning can be achieved using either doubled or tripled Nd-YAG lasers, both of which are readily available industrial tools.

Multiple switched areas have also been shown to be practical, and this opens the way for fabrication of arrays of smaller devices.

\section{\$2.4 Task 4 - IGU Durability and Integrity}

In order for the SageGlass ${ }^{\circledR}$ window product to meet the anticipated service requirements, it is necessary to ensure a durable and high quality seal to protect the sensitive EC films from the effects of moisture and physical damage, i.e. scratching. In addition, the electrical connection to the films must also be durable and cost effective, and provide a convenient means for connecting to the control system, by being compatible with the window framing system.

This task describes the work done on these fronts, first looking at the electrical feedthrough system, and then the overall device durability and reliability.

\section{\$2.4.1 Optimum Electrical Connection}

The EC IGU differs from conventional windows in that the interconnections to power the EC device must pass through the primary seal. The primary seal functions as a moisture barrier, and excellent performance is critical for long-term reliability of both the EC device and the IGU. It is therefore essential to develop an electrical connection method for the EC IGU that resists corrosion and does not compromise the moisture seal. In addition, the connection scheme developed must be manufacturable, low cost, and easily integrated into standard framing systems.

\section{\$2.4.1.1 Development of low cost feedthrough system}

SAGE has developed a proprietary low cost feedthrough system that is described in Section $\$ 2.4 .1 .1$ in the Appendix. The feedthrough system brings the electrical connection from the films inside the IGU to the outside.

\section{\$2.4.1.2 Durability results on low-cost feedthrough System}

Industry standard IGU seal tests were carried out in order to determine the integrity of the low cost electrical feedthrough. An argon diffusion test was carried out to determine the diffusion rate of the argon filler gas through the feedthroughs and sealing materials. The requirement for passing the argon retention test is less than $1 \%$ diffusion from the IGU per year. SAGE IGUs with the low cost feedthrough showed an average annual diffusion of $0.71 \%$. Samples with this 
IGU configuration also passed the P1 test, a French (soon to be European) standard weathering test, and a European humidity test, all of which are detailed in Section $\$ 2.4 .3$ below. These results have indicated that this low cost feedthrough approach is durable to water ingress and does not accelerate argon loss compared to standard insulating glass units.

\section{\$2.4.2 Development of a Lower Cost Method for Bus-Bar Deposition}

Two approaches were considered for the development of a low-cost approach to depositing busbars: direct dispense and electroplating. Both of these approaches have the potential advantage of reducing the cost of the process - compared with the current screen-printing technique - while reducing the possibilities for defect generation. Defects in the screen-printing process are caused by stray conductive material getting onto the active film area and leading to shorts.

\section{\$2.4.2.1 Directly Dispensed Bus-Bars}

The screen-printed bus-bars used in this work are deposited in a batch process, which is both wasteful of material and a bottleneck for the process. In addition, the process can cause contamination of the active area of the device unless extreme care is taken. The bus-bars are

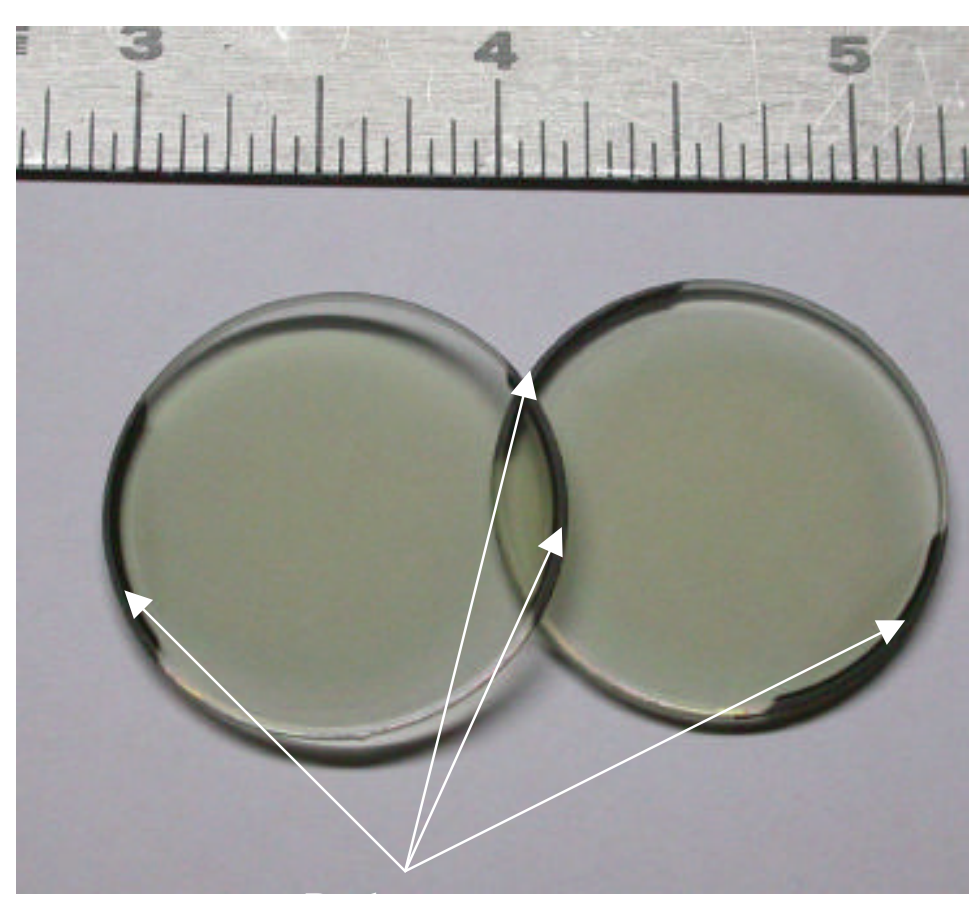

Figure 2. 26 - Small (3.2 cm (1.25”) diameter) EC devices fabricated using directly dispensed bus-bars. The bus bars are indicated by the arrows. deposited from a metallic paste and then heated to drive off the solvent. This means that it is easy to contaminate the screen with metal particles, and hence the surface of the glass, thereby leading to shorts in the finished EC device. Directly dispensing the conductive material onto the glass would eliminate the problem of potential screen contamination, hence the possibility of shorts.

It has been possible to directly dispense the present material using an instrument developed by Micropen Inc. ${ }^{* * *}$ Several smaller samples have been fabricated using this technique, and examples of finished devices are shown in Figure 2. 26. These samples were fabricated as part of a larger array, and cut up into individual devices after the EC film deposition had been finished. The bus-bars are the darker curved areas indicated by the arrows, and show that sophisticated patterning is possible.

\footnotetext{
*** Micropen Inc., 93 Paper Mill Street, Honeoye Falls, New York.: http://www.ohmcraft.com/micropen.htm.
} 
Larger area deposition - such as would be required for larger windows - is more difficult, as the particular equipment used for this work cannot supply the quantity of material necessary to produce the bus-bars economically. Further investigation into locating a suitable machine is currently on-going.

\section{\$2.4.2.2 Electroplated Bus-bars}

\section{Why plating?}

Bus bars on SAGE electrochromic devices serve to distribute voltage applied at contacts more uniformly. These have traditionally been applied by screen-printing, prior to deposition of the other layers making up the EC device. Problems with this approach include frit and metallic contamination defects in the device area, shorts due to pinholes in screens, and the need to apply bus bars before the device layers. The ability to apply bus bars after the device stack is completed would offer attractive device structure options. Plated bus bars could be applied in principle before or after the other device layers are deposited. Lower resistances are also available in solid metal conductors than in thick film materials, which would allow reduction in bus bar sizes, improving device vision area.

\section{Approach}

In order to quickly determine whether plating was a viable bus bar formation option, several routes to this knowledge were begun in parallel. Partnerships with two local commercial plating houses were begun. Contact was made with a University of Minnesota Chemical Engineering professor, who is an experienced electrochemist. An in-house literature search was carried out, and plating equipment and chemistry was obtained for SAGE trials.

\section{Literature}

A search was conducted for plating and plated layer adhesion to fluorine-doped tin oxide (FTO) and indium-tin oxide (ITO). No references to FTO were found, and only a scant few related to plating metals onto ITO were located. These all dealt with the experience of the liquid crystal flat panel display industry. Information gleaned from these sources indicated that generation of a sparse layer of reduced tin on the surface, followed by a palladium halide activation, gave the most conductive and adherent films. Extensions to FTO surfaces seemed a reasonable supposition.

\section{University Contact}

Professor W. Smyrl, University of Minnesota Department of Chemical Engineering and Materials Science, a noted electrochemist, was contacted concerning plating adhesion to FTO. He had previously encountered this issue in the course of unrelated research. No solution to what was, to him, a secondary problem was found, but he was able to tell us that the problem appeared to be related to some unknown variability in the condition or composition of the FTO surface, which lead to adequate adhesion in some regions, and almost no adhesion in neighboring regions of the same surface, after undergoing the same treatments. This was in exact agreement with the results from both platers and SAGE's internal work. The nature and source of these variations remains unknown. 


\section{Vendors}

Two commercial plating houses in the Minneapolis-St. Paul area worked with SAGE to provide a commercial perspective to possible solutions to this problem. Vendor "A" concentrated on preplating etches and cathodic reduction to enhance adhesion of plated copper films. Reduction of the surface seemed to be most successful, if not carried out too strongly, but the occasional successes were not reproducible. "A" quickly lost interest in the project and stopped all work.

Vendor "B" operated in parallel with "A", and was much more persistent, but ultimately, had little more success in producing strongly adherent, highly conductive, plated metal films on FTO. Again, occasional successes proved not to be reproducible, or to provide good adhesion only to a patchy portion of the surface, while the remainder of the film was readily removed by the "Scotch Tape Test", or even by merely wiping with a paper tissue. The most successful process at "B" was quite complicated: ammonium bifluoride dip > stannous fluoride dip > palladium chloride dip > nickel "strike" from an acid chloride bath > nickel plating from a sulfamate nickel bath. Plating current densities are important. Electroless plating was evaluated with even lower success. Nickel films with sheet resistivity of $2-3 \Omega$ /square could be obtained. Efforts to lower this value by thicker nickel plating, or by copper or gold over-plating, always resulted in delamination. A sheet resistance of $<1 \mathrm{~m} \Omega$ /square is required for optimum performance with large devices.

\section{SAGE Experiments}

Small-scale plating experiments were conducted at SAGE using Hunter, Inc., "Plating Pen" electroplating apparatus. Nickel, copper, and gold processes were investigated. A Hunter "Degreaser Pen" was evaluated to promote adhesion. The Hunter system of equipment is a labscale variation on the historic "brush plating" concept. A "plating pen" is constructed much like a wide felt tipped marker, with plating solution in place of ink, and electrodes to which to attach power supply leads. Voltage recommendations are supplied with the pens. This work early in this project lead to the same conclusions developed by the professional platers later on: cathodic reduction helps; nickel sticks to FTO much better than copper; attempts to plate nickel to less than $\sim 2 \Omega$ /square, or to plate copper onto nickel, result in peeling; and the results are always somewhat patchy. Consistent adhesion and low resistance were not obtained.

\section{Summary}

Results obtained from lab-scale experiments at SAGE and from two commercial plating houses were consistent in producing nickel films with sheet resistances of no less than $\sim 2 \Omega$ /square, which adhered well to some parts of FTO-coated substrates, but not to others. Treatment of the surface to produce tin in some reduced state, combined with palladium activation, produce the most adherent films over the greatest areas, but there are always considerable regions over which good adhesion is not achieved. Attempts to decrease resistivity by plating thicker nickel, or by adding copper or gold over layers, lead to delamination. The appearance of the results, and the comments of an academic consultant, lead to the conclusion that FTO surface inconsistencies of an unknown nature are responsible for the inability to achieve better results. A detailed study of the FTO surface that could resolve the mechanisms involved in metal adhesion is beyond the scope envisioned for this project, and, given the low level of success to date, no additional work is contemplated. 


\section{§2.4.3 Develop tests to ensure IGU Durability}

This sub-task dealt with development of testing aimed at stressing the IGU in ways that have so far been poorly addressed, such as thermal shock, and electrochemical corrosion of contacts. It is essential that the SageGlass ${ }^{\circledR}$ IGU is able to withstand all of the standard stresses seen by IGU, but also the stresses which are unique to electrochromic IGUs.

\section{\$2.4.3.1 Accelerated weathering and seal testing}

During the course of this project, a testing program has been developed in which IGUs with the SAGE sealing system and also fully operational SageGlass ${ }^{\circledR}$ IGUs have been subjected to industry standard tests, modifications of these standard tests and new tests. The test modifications have been designed to better stress this new IGU product. The key issues this test program is aimed to address are as follows:

- Integrity of the IGU sealing system with respect to water ingress into the air gap - does the feedthrough present a path for water?

口 Integrity of the IGU sealing system with respect to argon retention;

- Quality of SAGE's fabrication process;

口 Durability of the electrical connection, which is external to the spacer.

This testing program has been implemented with help from customers and partners and the program of tests that have been used and developed for SageGlass ${ }^{\circledR}$ durability evaluations, are:

- P1 test (constant UV irradiation at the seals and water spray at $60^{\circ} \mathrm{C}$ );

a The French IGU weathering test (includes thermal cycling, relative humidity and UV radiation) which will soon become the European standard;

European argon retention test;

․ The European humidity test;

- A customer's weathering test (UV, thermal cycling, one edge of IGU in water, humidity);

- More stringent, modified, version of US ASTM humidity test (developed in-house); and

․ Salt fog test (developed in-house).

Test results:

Argon retention test - This test was carried out by a customer-partner and the results are discussed in Section $\$ 2.4 .1 .2$ above on page 58 .

P1 test - The P1 test is used routinely by a major OEM IGU fabricator, and is believed to be the most stringent weathering test, more so than the ASTM weathering test (ASTM 774). They have found a correlation showing 1-week-in-test to be equivalent to 1-year-in-the-field and set the pass requirement in this test at 40 weeks. Initially, units were tested with the SAGE feedthrough system with and without SageGlass ${ }^{\circledR}$ films, both of which successfully surpassed the 40 week requirement. These results indicate the durability of the SAGE sealing system and SAGE's fabrication quality. Next, to test the durability of the electrical connections outside the primary seal, full SageGlass ${ }^{\circledR}$ IGUs were electrochromically cycled (to ensure the connections have an electrical potential across them) while under the P1 stress. Five of five units survived 58 weeks 
in the test while accruing approximately 30,000 EC cycles. All are fully operational as EC devices, pass the dew-point requirements indicating no seal failure, and the electrical connections show no sign of performance degradation.

European tests - A customer-partner has carried out the French weathering test, European humidity test, and customer's weathering tests on IGUs with the SAGE feedthrough and sealing system. The positive results support the indications from P1 test results that both the sealing system and SAGE's fabrication quality are good.

Modified humidity test - An in-house humidity test was designed and built. This is thought to be a more stringent version of the ASTM humidity test for IGUs, because the test temperature is increased from 60 to $75^{\circ} \mathrm{C}$ and has one edge of the IGU immersed in liquid water while the remaining atmosphere is close to $100 \%$ relative humidity. Depending on the test, the end of the IGU with the electrical connections can be immersed in the water. In addition to the environmental stresses, the electrical connections are further stressed by continually cycling the devices from clear to dark to clear. Altogether, 22 units underwent eight months of EC cycling with contacts in water, 16 of which exhibited no change in electrical system performance (no impact of corrosion). The other six samples exhibited seal failures due to the humidity stress. It should be noted that the ASTM standard humidity test requires units to survive only 14 days in $95 \% \mathrm{RH}$ and $60^{\circ} \mathrm{C}$.

Salt Fog - an even more highly accelerated test was initiated - using a salt fog chamber - to investigate the potential for corrosion of the EC electrical connections, which are located outside the air gap, under the secondary seal. In this test, just as with the P1 and in-house humidity test, the SageGlass ${ }^{\circledR}$ IGUs were electrochromically switched while exposed to highly concentrated salt water ( 5 times that of sea water) at $40^{\circ} \mathrm{C}$. The resistances of the electrical connections were monitored as a function of time, to determine any loss of electrical performance due to corrosion. After four months in the test, seven of the original 10 devices were still operational without electrical system failure. Three devices failed because silicone secondary seal coverage was not sufficient around the spacer - salt attacked the aluminum spacer causing a hole and loss of desiccation inside the IGU. Note that this test is simply a pass/fail test, and therefore duration in the test prior to failure is not representative of anything other than how long the corrosion takes to cause failure, which is a function of the rate of the corrosion reaction and the original size of the hole in the sealant.

\section{Summary}

The above results indicated that there are minimal issues associated with failure of the electrical connections in the SageGlass ${ }^{\circledR}$ IGU configuration as long as they are covered completely with silicone. At this point in the test program implementation, the electrical configuration and IGU seal looks durable to the expected environmental stresses.

\section{\$2.4.3.2 Impact of thermal shock on EC IGUs}

When a SageGlass ${ }^{\circledR}$ IGU is in its dark state under direct sun, it absorbs significant thermal energy and consequently gets hot. If a sudden rain-storm occurred, the water would cause the glazing to cool very rapidly inducing a thermal shock. Also, if the glazing is very cold, (e.g. during the winter in northern climates), and the glazing suddenly comes under direct sun, while in the dark state it will absorb energy and the temperature will rise rapidly, again resulting in thermal shock. Even commercially available tinted glazings in use today have visible light 
transmissions significantly higher than that of the dark state of SageGlass ${ }^{\circledR}$ glazings so the rate of change of temperature of these glazings under the same conditions is likely to be significantly less. Clearly, not only will thermal shocks cause stress to the coatings, they will also stress the film to bus bar connections and the IGU sealing system itself.

To evaluate the durability of the SageGlass ${ }^{\circledR}$ IGU system to thermal shocks, SAGE has developed a test system that simulates and exaggerates the thermal shocks that a window would experience in the actual environment. Briefly, the SageGlass ${ }^{\circledR}$ IGU is mounted in a metal frame directly in front of an array of solar simulation lamps. The test is located outside, so that the temperature of the glazing varies with the outside ambient temperature. In a given transmission state, the window can be suddenly illuminated by irradiation of intensity greater than 1 sun (AM 1.5). An automatic shower can be triggered to simulate the thermal shock of rain. Masks can also be placed over portions of the glazing to simulate partial shading. Thermocouples are placed over the front and back surfaces of the glazing to measure temperature gradients.

Over the course of 15 months, one SageGlass ${ }^{\circledR}$ IGU survived more than 3,000 thermal shocks, 770 of which included rapid cooling, without seal failure or EC failure. The largest cooling rates measured were $140^{\circ} \mathrm{C} /$ minute, with a $56^{\circ} \mathrm{C}$ temperature difference between the front and back lite.

The equipment was only capable of delivering around 5 thermal shocks per day to a single glazing, so 3000 shocks represents more than a years worth of operation, i.e. essentially the entire project duration. In short, only two samples were tested, the one for which the results are reported above, and a second instrumented IGU - constructed with thermocouples attached to various sealing surfaces. This IGU allowed us to measure the seal temperatures, but the seals were necessarily compromised by the TCs feeding through, so this unit was not considered for inclusion in the durability study.

In order to make the test a better representation of a real environment the test was modified so that the glazing was glazed into an air conditioned box, keeping the inside lite of the IGU at a constant room temperature (as it would be in a real installation) while the outside EC lite was illuminated. Temperature gradients and cooling and heating rates have been observed to be significantly reduced as a result of this modification, indicating that the original test was indeed very highly accelerated.

The one passing unit actually experienced a significant number of shocks under both unmodified and modified conditions. The history was as follows:

1. Equipment set up;

2. Thermal shock testing initiated on glazing;

3. Equipment modified

4. Shock testing continued on same glazing.

Glazing passed 3000 shocks without fail. 
Dependence of seal temperature on COG temperature - 25 June 2003. Second day after screen removed to increase intensity of illumination incident on glass. Inside held close to $20 \mathrm{deg}$.

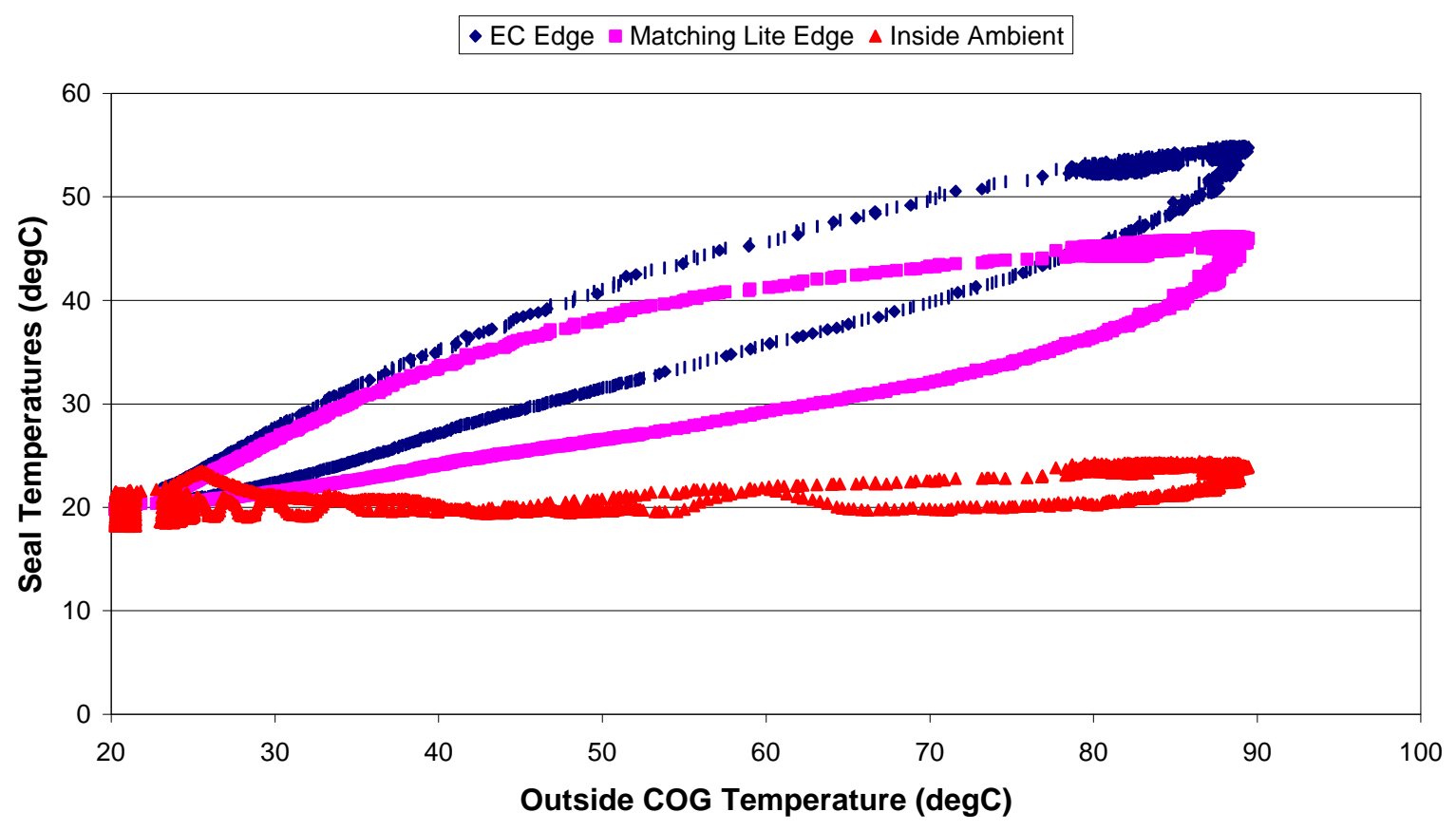

Figure 2. 27 - Measurements of seal temperatures of a SageGlass ${ }^{\circledR}$ IGU in the outdoor solar soaker, plotted as a function of the center-of-glass (COG) temperature of the EC lite. Note that even for temperatures of $90^{\circ} \mathrm{C}$, the seal temperatures only reach about $60^{\circ} \mathrm{C}$, suggesting that even for these high exposures the seals should be capable of surviving.

The temperatures measured using the embedded thermocouples are shown plotted against the temperature measured on the outside center-of-glass (COG). Also plotted is the temperature of the inside ambient that is being controlled (using a small air conditioning unit) to $20^{\circ} \mathrm{C}$. From these data it is clear that despite the COG temperature reaching close to $90^{\circ} \mathrm{C}$, the seal temperatures do not exceed $60^{\circ} \mathrm{C}$. The measurement of the simulated solar intensity showed that the illumination was equivalent to one sun at AM1.5, so it is a realistic test for the window, although it is likely that some windows in more southern climates may see slightly higher solar intensities.

\section{\$2.4.4 Solar Simulator Testing}

Several samples have been subjected to third party solar simulation testing at The National Renewable Energy Laboratory (NREL) in Golden, Colorado ${ }^{\dagger \dagger \dagger}$. These devices were sent to NREL for solar exposure in the XR-260 solar simulator.

Prior to insertion in the XR-260 chamber, the devices were electro-optically characterized at room temperature and $85^{\circ} \mathrm{C}$ using electrical protocols agreed upon by NREL and SAGE. Cyclic

†† NREL, 1617 Cole Boulevard, Golden, CO 80401 
environmental testing of these devices was accomplished using methodology outlined in ASTM standard E2141-02.

\section{Test Conditions and Procedures}

NREL's Atlas XR-260 large component, environmental test chamber (see Figure 2. 28) provides simulated solar irradiance using four 6500 watt, water-cooled, long-arc xenon lamps mounted in

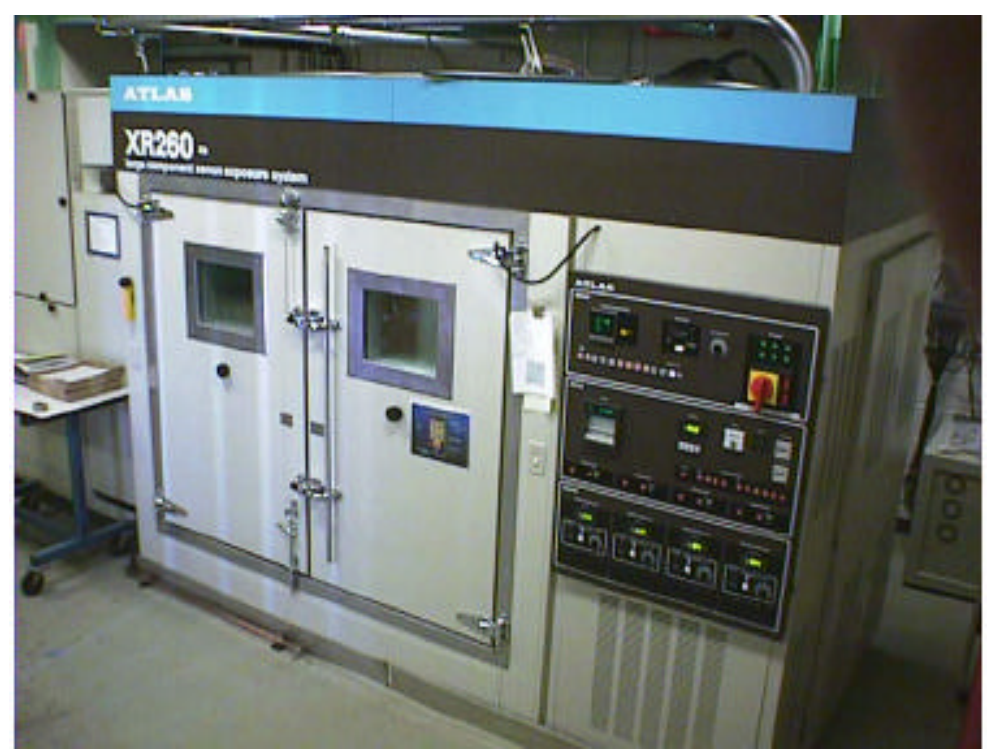

Figure 2. 28 - The XR260 test chamber at NREL. the ceiling above a heightadjustable $1 \times 2 \mathrm{~m}\left(4^{\prime} \times 6\right.$ ') sample plane. The xenon lamps are spectrally filtered (CIRA inner filter, soda lime outer filter) to provide precise solar cut-on and an excellent spectral match to an air mass (AM) 1.5 global solar spectrum from 300 to $800 \mathrm{~nm}$. At longer wavelengths, the fit to the solar spectrum is compromised by xenon emission lines, which are unimportant for these test purposes because they generally do not contribute to photo-induced degradation. Prior to the start of testing, new xenon lamps were installed; the sample plane was optically mapped; and the XR-260 electronic controls were calibrated.

In this test, the following experimental settings in the XR-260 were used under manual control to achieve the nominal sample temperatures and a 1.1 sun equivalent of an AM 1.5 global solar spectrum across a band width of 300 to $400 \mathrm{~nm}$ (the spectral region which is likely to cause most degradation of the devices):

Dry bulb temperature $(\mathrm{DBT})=35^{\circ} \mathrm{C}$

Black panel temperature $=51^{\circ} \mathrm{C}$

口 Lamp Power $=5.8$ to $6.6 \mathrm{~kW}$

口 Irradiance at $340 \mathrm{~nm}=120 \mathrm{~W} / \mathrm{m}^{2}$ (for each lamp)

․ Sample plane height $=78.2 \mathrm{~cm}$

口 Relative humidity $=6$ to $9 \%$ (ambient)

The four-inch long breather tube emanating from the corner of each IGU window was secured to the edge of each device with aluminum tape. This procedure ensured that the tubes were protected from inadvertent damage when handling the devices. Similar tape was used to mask the electrical leads.

Each of the EC-IGU windows was outfitted with a $0.013 \mathrm{~cm}(0.005$ ") diameter Type-K thermocouple pasted to the top center surface with an $\sim 8 \mathrm{~mm}$-square patch of aluminum tape. 
Temperatures of the EC devices ranged from 68 to $78^{\circ} \mathrm{C}$ (with an average of $73^{\circ} \mathrm{C}$ ) depending on their position within the XR-260 chamber.

Measurements of the bleached state transmittance, colored state transmittance and Photopic Transmittance Ratio (PTR) were taken at various convenient points when the cycling was interrupted. Transmittance data were measured at a wavelength of $550 \mathrm{~nm}$, which represents the peak of the photopic spectrum. An example of room temperature electrical characterization curves (current vs. time) for zero; $7 \mathrm{~K}, 14 \mathrm{~K}, 24 \mathrm{~K}, 34 \mathrm{~K}, 44 \mathrm{~K}, 54 \mathrm{~K}, 64 \mathrm{~K}$ and 80,000 cycles of testing are shown in Figure 2. 29. Very little change is seen as a result of the test, indicating excellent stability to solar exposure.

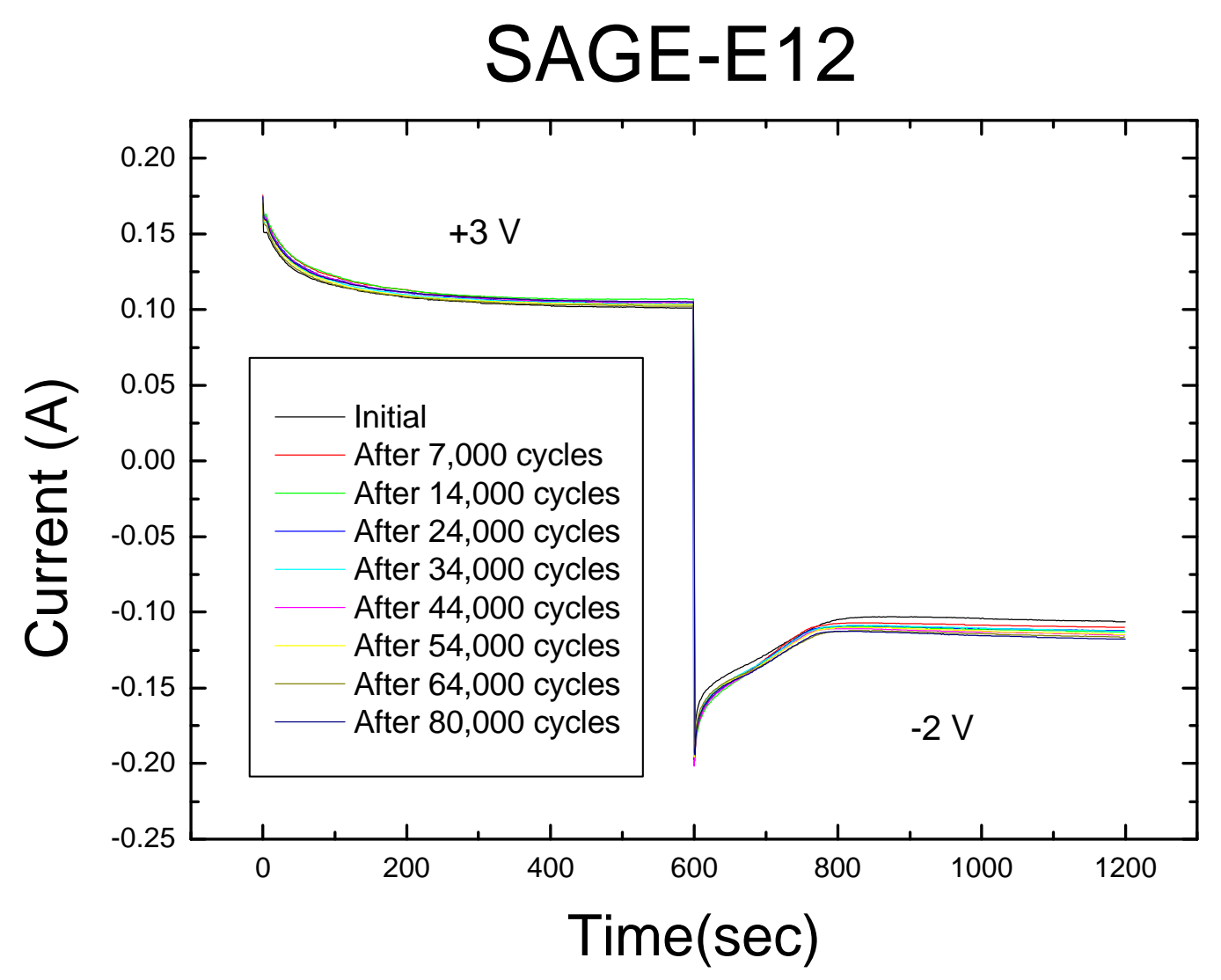

Figure 2. 29 - Charge discharge curves for one of the SageGlass ${ }^{\circledR}$ IGUs subjected to simulated solar soaking at NREL, measured at various stages of cycling. It is apparent that there is very little change in the characteristic as a result of the solar soaking.

Figure 2. 30 shows transmittance vs. wavelength curves for one of the devices after initial and $80 \mathrm{~K}$ cycle characterization. The following notes and general trends were evident from the measured data as well as from visual observations of the devices:

Optical Characterization after 80,000 Cycles: 
Both the bleached and colored state conditions upon removal from the XR-260 environmental chamber of all three EC-IGU windows were carefully examined. The average photopic transmittance remained virtually constant throughout testing for both states.

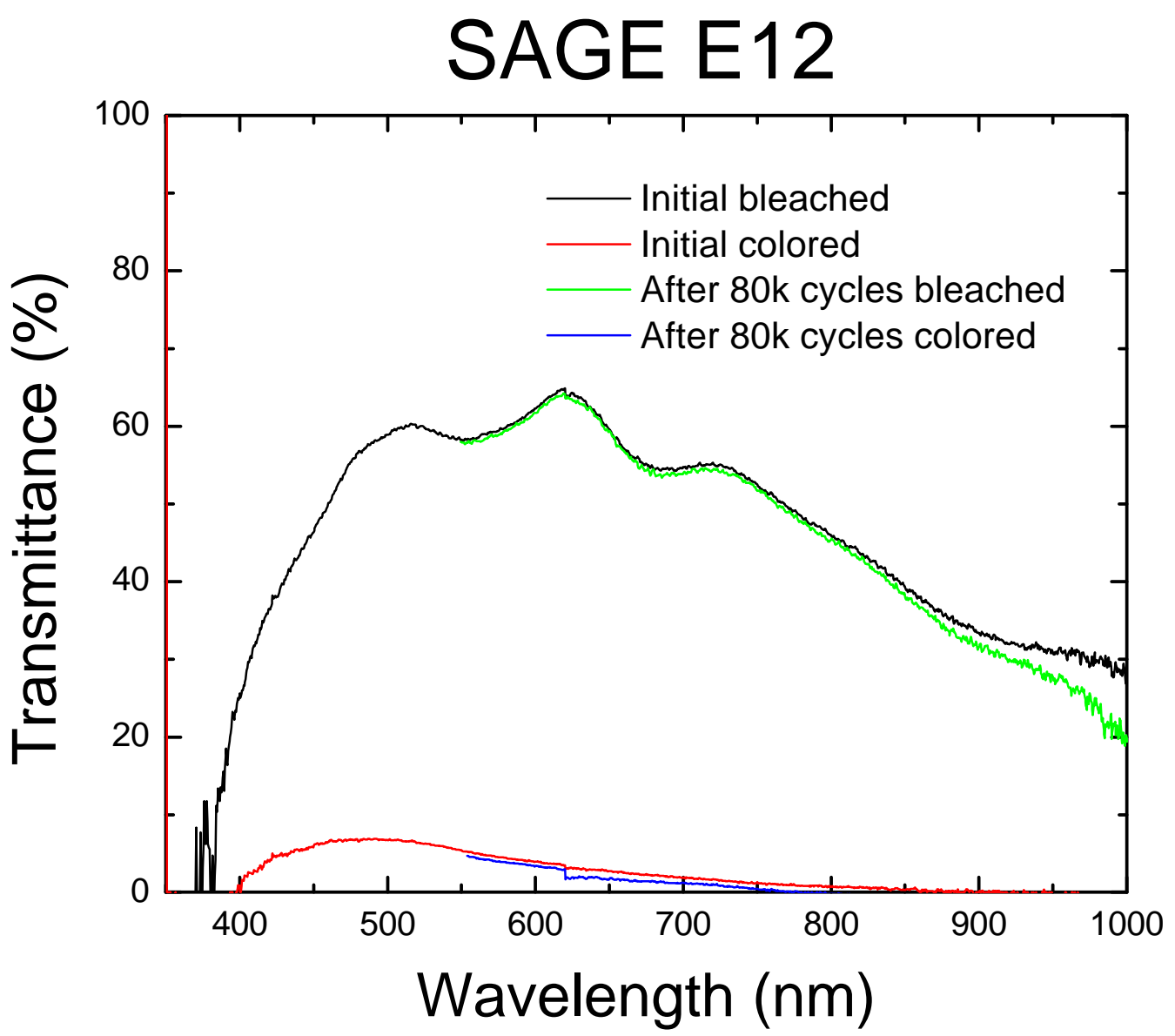

Figure 2. 30 - Transmission as a function of wavelength for one of the devices subjected to simulated solar soaking at NREL measured at the start of the test, and after $\mathbf{8 0 0 0 0}$ cycles. Once again, it is clear there is very little change as a result of the testing.

Electrical Characterization After 80,000 Cycles:

In general, the devices exhibited only minor differences in their color ( 3 volt, $10 \mathrm{~min}$.) and bleach (-2 volt, 10 min.) curves throughout the test. These electrical data basically support optical characterization data for the devices, which indicate little change in device performance throughout the test.

The charge/discharge capacity data taken during testing also indicate no basic changes in device performance. Also, the devices do not appear to have lost any kinetic performance when compared to their original ion-insertion characteristics. Overall, all the EC windows continued to 
exhibit deep uniform coloration and remarkable durability with no appearance of any new cosmetic aberrations across 80,000 cycles of testing.

\section{Summary:}

The SAGE EC-IGU windows accumulated a total of $\sim 80,000$ cycles of accelerated environmental testing under ASTM Standard E2141-02. This standard is designed to evaluate the combined effects of heat, solar AM 1.5 light (1.1 UV sun equivalent) and extended electrical cycling. In general, all the devices still functioned with a high bleached state transmittance, high photopic transmittance ratio, little change in electrical characteristics and a relatively good visual appearance devoid of major cosmetic irregularities at the conclusion of the test. Visual observations and photopic transmittance data in the bleached state indicate no obvious degradation. When compared to initial values, bleached state transmittances of the devices remained relatively unchanged while colored state transmittances averaged over a number of devices increased slightly from 5.2\% to 5.7\%. The characteristic in Figure 2. 30 show data from a device where the colored state transmission remained fairly constant. This is generally more characteristic of recent devices, and so is shown here.

\section{§3. Discussion and Conclusions}

This project has addressed several aspects of EC window production, with a particular emphasis on manufacturability. It is evident from the preceding technical discussion that much has been achieved. The content of the program was wide ranging, incorporating microscopic studies of defect mechanisms, as well as device testing, hardware development, quality programs, and theoretical modeling. Many useful things have been learnt as a result of carrying out this project. For example, impressive durability results have been obtained for the EC IGU.

The following summarizes the high level results from each of the tasks in turn, highlighting the major achievements for each.

\section{Task 1: Materials Development}

- Investigated the electrochemistry of a mixed oxide EC layer and found it to be acceptable for EC device fabrication;

- Successfully deposited EC films from a ceramic target, but could not fabricate a functioning device using such films. However, this work did provide a valuable insight into the EC film properties required for working EC devices;

- Produced functional devices through carefully controlled sputtering of near metal mode films;

- Investigated different component ratios in the $\mathrm{CE}$, and increased the understanding of device behavior and dependence on CE properties;

- Thoroughly researched the effect of poorly controlled process conditions, such as background gas levels, substrate temperature prior to deposition, magnetic array strength and uniformity, etc., on the properties of the CE films in particular, resulting in dramatically improved repeatability and therefore improved quality for the process;

- Produced a second generation ion conductor with dramatically improved performance;

- Developed a solid-state model of the EC device; 
- Improved the understanding of the rate limiting factors for switching speed in the EC device.

\section{Task 2: Process Improvement}

- Developed a new heating process which eliminated the 'rib' defect;

口 Dramatically improved uniformity of heating.

\section{Task 3: Operational Testing}

- A custom EC test station has been designed, built and the control software written. The tester allows measurements of electronic and optical performance of multiple devices simultaneously, and is networked so data added to a database can be remotely accessed and analyzed;

- An electro-optical device model has been developed, as the first step towards allowing extraction of a set of parameters to calibrate a device controller;

- Design of Experiments methodology has been introduced across the entire company in order to maximize the benefits of the limited experimental time which is available;

L Laser patterning has been shown to be an effective way of producing the line isolation for the process.

\section{Task 4: IGU Durability and Integrity}

- A low-cost, highly reliable electrical feedthrough system has been developed to enable the power to reach the EC device without compromising the IGU seal;

- Methods for investigating a lower cost bus-bar deposition process have been pursued, with limited success. Attempts to produce an electroplated bus-bar have been unsuccessful, but direct dispense methods show promise, not only for lowered costs, but also for producing lower defect levels;

- Tests to measure the durability of the IGU have been developed;

口 The SageGlass ${ }^{\circledR}$ EC IGU have demonstrated excellent durability to a wide variety of physical, electrical and chemical stresses;

\section{§3.1 Achievement of Project Objectives}

The overall objective of the project was to demonstrate the viability of EC technology for energy efficient architectural glazing applications. It was focused on improving the EC performance durability, increasing the manufacturability and therefore the yield, thereby reducing the cost to allow high market penetration. Achieving this would accelerate the widespread acceptance of EC technology in US buildings. More specifically, the scope was to:

- Develop and implement new materials technologies to improve EC glazing performance and enable broader penetration of both residential and commercial markets;

- Develop new EC process technology to increase yield and throughput, resulting in reduced manufacturing cost; 
- Assure EC reliability and durability by developing robust fabrication methods and implementing extensive environmental testing of both the EC IGU and the thin-film device.

Improvements of glazing performance have been achieved by incremental improvements to many different sub-processes within the overall manufacturing scheme. These have occurred continually, and it is therefore difficult to assign significant improvements seen in the measurements to particular events. Nevertheless, by reference to the following (Figure A3. 1, Figure A3. 2, Figure A3. 3 and Figure A3. 4) it is clear that significant improvements have been achieved in several of the key performance parameters.

Figure A3. 1 shows the dynamic range of production devices - those devices not involved in any process parameter experimentation - of the larger sizes, i.e. $46 \mathrm{~cm} \times 89 \mathrm{~cm}(18 " \times 35$ ") or $48 \mathrm{~cm} \times 94 \mathrm{~cm}(19 " \times 37$ "). The dynamic range is calculated from the colored and clear state transmission. Improvements in dynamic range reflect either an increase in the clear state transmission, or a decrease in the colored state transmission, both of which are desirable. (Note: the transmission measurements were taken using standard conditions throughout the period of the project). In this case, the improvement is a result of improvements in both parameters.

One of the targets of the original proposal was to increase the clear state visible light transmission of the SageGlass ${ }^{\circledR}$ window - i.e. the IGU - to a value of $70 \%$. At the beginning of the project, this parameter was typically $60 \%$. As a reference, a standard low-e product used in residential installations today has a visible light transmission of between 70 and $75 \%$. This places the goal of $70 \%$ visible light transmission for the SageGlass ${ }^{\circledR}$ IGU in context. This extremely difficult goal has not been achieved, but significant progress has been made towards it. Currently, SageGlass ${ }^{\circledR}$ IGUs are fabricated with clear state visible light transmission between 57 and $62 \%$, with the range representing some process tolerance, and some variability due to the experimental nature of the mode of operation of the Pilot Line. The small increase in clear state transmission is largely a result of improvements in overall process control and in particular improvements in uniformity and repeatability, and not as a result of any major process changes. It has been determined as a direct result of work done in this project that an increase in bleached state transmission can be attained if the composition of the CE material is adjusted. This is likely to improve matters by several percent, without compromising any of the other properties of the device, but it has yet to be fully implemented at the Pilot line stage. Furthermore, work done outside the project has shown that anti-reflecting capping layers can increase the clear state transmission by several percent, currently giving clear state transmission of up to $66 \%$. It is clear that the efforts to improve the clear state transmission investigated during this program have formed the foundation for further improvements, which are likely to pay off in the near future.

Figure A3. 2 shows the electronic leakage current in the colored state plotted as a function of time. Lower leakage current is better, and clearly there has been a trend to lower currents during the course of the project.

Finally, Figure A3. 3 and Figure A3. 4 shows the improvements in uniformity for the clear and colored states obtained during the project. The uniformity is measured as $\ddot{A} E$, where

$$
\Delta E=\sqrt{\Delta L^{* 2}+\Delta a^{*^{2}+\Delta b} *^{2}}
$$


and $L^{*}, a^{*}$ and $b^{*}$ are the well known CIE color co-ordinates, and the $\ddot{A}$ represents the range of the parameter measured. Once again, it is clear that the overall trend is to better uniformity, both in the colored and the clear states.

Interestingly, a process change can sometimes be beneficial to one parameter - say for example, a reduction in the leakage current - but detrimental to another seemingly unrelated parameter say, the bleached state transmission. Improvements have to be judged in the context of the whole device performance, and therefore offer a compromise. For example, it is possible to reduce the residual absorption seen in the clear state by reducing the thickness of either the $\mathrm{CE}$, the top TCO or both. However, this would cause a reduction in the depth of coloration, and also would significantly affect the uniformity of the device during switching and in the colored state.

The understanding of the relationships between various device characteristics has been significantly improved by the work done in this project, allowing the necessary compromises to be made in order to produce the device that best satisfies the market requirements.

Overall, it can be said that the materials improvements made as a result of the work done during this project, however incremental, have contributed to the improvement seen in a variety of different parameters, including dynamic range, uniformity and electrical characteristics.

The second major objective of the project was to develop technology to improve yield, reduce cost, and facilitate manufacturing of EC products. The improvements seen in the overall EC device performance have been accompanied by an improvement in the repeatability and consistency of the production process. This is evident in the reduction of the scatter in the data, and is naturally reflected in an improvement in the yield, which in-turn leads to a reduction in cost. Actual values of yield depend on comparison of the measured data with a specification, and as this is a new product, the specification will, to some extent, be determined by market acceptance and pre-launch trials. For this reason, actual yield numbers are not available.

With regard to improving manufacturability, the most notable effort has entailed development of test facilities for characterizing devices in a timely and well-defined manner. The equipment has been designed in such a way as to make scaling-up to accommodate higher throughput relatively straightforward, and is clearly something that is ready for the next phase of implementation transfer to manufacturing.

Although the original proposal targeted an increase in testing velocity by determining key performance indicators as a result of the development of a device model, it was found to be necessary to fully color and bleach the device to determine the occurrence of point defects, and coloration uniformity, color, etc. This really sets the limit on the time that each window requires for testing, and this is dependent on the size - the larger devices switch more slowly. This project has resulted in the design and construction of several testers, each of which takes a significant quantity of data required for determining whether the device passes the performance specification. Each tester is also capable of testing up to two samples in parallel, so it will simply be necessary to construct the requisite number of test stations to accommodate the throughput expected during production. One further improvement to the process will be the automating of the system to remove the requirement for full-time human supervision of each test station. In principle, this is not difficult to achieve, as the present tester already has a camera used for taking uniformity data, and a computer for recording and displaying the results. It is a simple matter to allow the computer to make pass-fail determinations on transmission levels, uniformity, etc, and to provide a computer controlled marking system for indicating the locations of point defects, 
which can subsequently be removed using a computer controlled laser. Given this parallel approach, it is certainly possibly to allow the testing function to keep pace with the production process.

One of the stated tasks in the proposal was to provide the manufacturing potential to achieve a certain selling price. Obviously, there are many factors involved in determining the actual costs and therefore selling price, including yields, throughput, overheads, etc. Clearly, a complete cost analysis prior to the start of manufacturing would be full of assumptions and approximations, and also well beyond the scope of this report. However, it is fair to say that much of the work done during this project has gone a significant way towards defining processes and equipment requirements, which in themselves will determine the costs of operating any particular process, and ultimately contribute to the overall product cost. Many, if not all of the tasks discussed in this report, are geared towards enabling the manufacturing plant to be set up. For example, the custom EC test station has been developed with a view to implementation in the manufacturing environment. Similarly, laser line isolation has been developed as a technique to potentially replace the messy sandblasting operation. As SAGE moves further into the manufacturing stage, it will become evident how successful each of these contributions has been, but the fact that SAGE is moving to the manufacturing stage is an indication of the success to this point.

Finally, the third major goal was to assure the durability of the EC product. This has quite clearly been demonstrated by a number of different durability tests, both in-house and carried out by independent third-party testers. Tests included solar soaking, exposure to high levels of humidity, temperature cycling, thermal shock testing, amongst standard glazing tests, as well as a range of new tests necessary to qualify the EC part of the system.

\section{\$3.2 Maturation stages and success criteria}

Table 1. 2 shows the task layout for this project in terms of the maturation steps. Task 1 , the materials development section, has a number of components. It is clear from the preceding discussion, that the overall EC process is ready to move to the manufacturing stage. Despite the fact that several of the individual aspects that were investigated, e.g. use of an oxide target, did not produce results good enough to justify moving to the next stage. Obviously, the current materials technology will therefore form the basis for the first all thin film EC production facility.

In general, the same can also be said for the process improvements, where the operational testing aspect and laser processing of line isolation have been completed successfully, while the tasks related to rapid thermal processing, while yielding a great deal of useful information, have not resulted in totally successful implementation.

Finally, however, the tasks related to durability have been overwhelmingly successful, both in terms of developing new durability tests, as well as improving the durability of the EC IGU product to the point where it is ready to be launched.

\section{§3.3 Concluding Remarks}

This project has been highly successful. The results have placed SAGE Electrochromics in a position to be able to confidently face the next phase of development, that of constructing a 
manufacturing facility. Developments made under this program will play a key role in underpinning the technology for producing EC devices.

It is anticipated that the work begun during this period will continue to improve materials properties, and drive yields up and costs down, increase durability and make manufacture simpler and more cost effective. It is hoped that this will contribute to a successful and profitable industry, which will help reduce energy consumption and improve building comfort worldwide. 


\section{§4. List of Abbreviations}

The following is a list of abbreviations used throughout the text.

\begin{tabular}{|c|c|}
\hline AAS & Atomic Absorption Spectroscopy \\
\hline $\mathrm{AM}$ & Air mass \\
\hline ANOVA & Analysis of Variance \\
\hline $\mathrm{CE}$ & Counter-Electrode \\
\hline $\mathrm{CV}$ & Cyclic Voltammogram \\
\hline CVD & Chemical Vapor Deposition \\
\hline $\mathrm{DC}$ & Direct Current \\
\hline DOE & Design of Experiments \\
\hline DOE & Department of Energy \\
\hline $\mathrm{EC}$ & Electrochromic \\
\hline ESCA & Electron spectroscopy Chemical Analysis \\
\hline EVOP & Evolution of Parameters \\
\hline FTO & Fluorine doped Tin Oxide \\
\hline $\mathrm{IC}$ & Ion Conductor \\
\hline IGU & Insulated Glazing Unit \\
\hline ITO & Indium Tin oxide \\
\hline $\mathrm{I}-\mathrm{V}$ & Current - Voltage \\
\hline LBNL & Lawrence Berkeley National Laboratory \\
\hline MOS & Metal Oxide Semiconductor \\
\hline NREL & National Renewable Energy Laboratory \\
\hline OD & Optical Density \\
\hline OFAT & One Factor at a Time \\
\hline PVD & Physical Vapor Deposition \\
\hline $\mathrm{sccm}$ & Standard cubic centimeters per minute \\
\hline SEM & Secondary Electron Microscopy \\
\hline SIMS & Secondary Ion Mass Spectroscopy \\
\hline TC & Transparent Conductor \\
\hline TCO & Transparent Conducting Oxide \\
\hline TEM & Transmission Electron Microscopy \\
\hline XPS & X-ray Photoelectron Spectroscopy \\
\hline
\end{tabular}




\section{§5. References}

${ }^{1}$ Buildings Energy Databook, 2002. US Department of Energy, Office of Energy Efficiency and Renewable Energy, htttp://buildingsdatabook.eren.doe.gov

${ }^{2}$ C.G.Granqvist, Crit. Rev. Solid State Mater. Sci. 16, 291-308 (1990); C.M.Lampert, Solar Energy Materials, 11, 1-27 (1984)

${ }^{3}$ For more details on this see: http://www.sage-ec.com

${ }^{4}$ E.S.Lee, D.L.DiBartolomeo, F.M.Rubinstein, and S.E.Selkowitz, 'High Performance Commercial Building Systems - Low-cost Networking for Dynamic Window Systems', Submitted to Energy and Buildings, August 2003.

${ }^{5}$ C.G.Granqvist, 'Handbook of Inorganic Electrochromic Materials', Elsevier (1995), Chapter 1 p1

${ }^{6}$ See for example C.G.Granqvist, 'Handbook of Inorganic Electrochromic Materials', Elsevier (1995) Chapter 13 p225

${ }^{7}$ Faughnan and Crandall, Appl. Phys. Lett. 31, 834-836 (1977).

${ }^{8}$ Yamada and Kitao, SPIE Vol IS4 pp246-259, (1990).

${ }^{9}$ M.S.Burdis, Thin Solid Films, 311 (1997) 286-298

${ }^{10}$ U.Opara Krašovec, A. Šurca Vuk, and B.Orel, Electrochemica Acta 46 (2001) 1921-1929

${ }^{11}$ M.S.Burdis and J.R.Siddle, Thin Solid Films, 237 (1994) 320-325

12 This microstructure is typical in materials in which $\mathrm{T}_{\mathrm{s}} / \mathrm{T}_{\mathrm{m}}\left(\mathrm{T}_{\mathrm{s}}=\right.$ substrate temperature; $\mathrm{T}_{\mathrm{m}}=$ film material melting temperature) is low as is the case here. The role of sputtering conditions on structure is described in a number of review articles on sputtering (e.g. H. Windischmann, Critical Reviews in Solid State and Materials Sciences 17 (6); 547-596 (1992)).

${ }^{13}$ C.G.Granqvist, 'Handbook of Inorganic Electrochromic Materials', Elsevier (1995), Chapter 27, p453

${ }^{14}$ CIE 1976 Chromaticity diagram.

${ }^{15}$ A.J.Bard and L.R.Faulkner 'Electrochemical Methods', Pub: Wiley (1980), Chapter 6

${ }^{16}$ S.M. Sze, 'The Physics of Semiconductor Devices', Wiley (1969)

17 A.S. Grove, 'Physics and Technology of Semiconductor Devices', Wiley (1967) p263

${ }^{18}$ K.L. Chopra, 'Thin Film Phenomena', p 479, McGraw-Hill (1969)

19 "DOE Simplified," by Anderson and Whitcombe (2000) Pub: Productivity Inc.

20 "Design and Analysis of Experiments," Montgomery, (1997), Pub. Wiley 